\title{
Multimodality Imaging of Silica and Silicon Materials In Vivo
}

\section{pÿ^en, Karaman Didem}

2018-06-13

pÿ^en , K D , P. , S M , M. , R J \& J. , A A 2018 , ' Multimodality Imaging of Silica and Silicon Materials In Vivo ' , Advanced Materials , vol. 30 , no. 24 , 1703651 . https://doi.org/10.1002/adma.201703651

http://hdl.handle.net/10138/328163

https://doi.org/10.1002/adma.201703651

unspecified

acceptedVersion

Downloaded from Helda, University of Helsinki institutional repository.

This is an electronic reprint of the original article.

This reprint may differ from the original in pagination and typographic detail.

Please cite the original version. 


\section{Multimodality Imaging of Silica and Silicon Materials in Vivo}

Didem Şen Karaman, Mirkka P. Sarparanta, Jessica M. Rosenholm* and Anu J. Airaksinen*

Didem Şen Karaman, Pharmaceutical Sciences Laboratory, Faculty of Science and Engineering, Åbo Akademi University, BioCity, Tykistökatu 6A, Fl 20520 Turku, Finland.

Dr. Mirkka P. Sarparanta, Department of Chemistry - Radiochemistry, University of Helsinki, POB 55, FI-00014 University of Helsinki, Finland

Prof. Jessica M. Rosenholm, Pharmaceutical Sciences Laboratory, Faculty of Science and Engineering, Åbo Akademi University, BioCity, Tykistökatu 6A, Fl 20520 Turku, Finland. Email: jerosenh@abo.fi

Prof. Anu J. Airaksinen, Department of Chemistry - Radiochemistry, Faculty of Science, University of Helsinki, POB 55, FI-00014 University of Helsinki, Finland, Email: anu.airaksinen@helsinki.fi

\section{Abstract}

Recent progress in the development of silica $\left(\mathrm{SiO}_{2}\right)$ and silicon ( $\left.\mathrm{Si}\right)$ based multimodality imaging nanoprobes has advanced their use in image-guided drug delivery and development of novel systems for nanotheranostic and diagnostic applications. As biocompatible and flexibly tunable materials, silica and silicon provide excellent platforms with high clinical potential for the development of nanotheranostic and diagnostic probes with well-defined morphology and surface chemistry, yielding multifunctional properties. In vivo imaging has proven to be of great value in the exploration of methods for improving site-specific nanotherapeutic delivery by silica and silicon based drug delivery systems. Multimodality approaches have been essential for understanding the biological interactions of nanotherapeutics in the physiological environment in vivo. The aim of this progress report is to describe recent advances in the development of in vivo imaging tools based on nanostructured silica and silicon, and their applications in single and multimodality imaging. 
Key words: image guided drug delivery, nanotheranostics, in vivo imaging, PSi, MSN

\section{Introduction}

\subsection{Image-guided drug delivery and nanotheranostics}

Nanomaterials have been investigated for biomedical applications since the early 1990s. However, the concept of a "magic bullet" was introduced even earlier, referring to a drug delivery system (DDS), which could selectively deliver a toxic drug into its target, thus minimizing the side effects caused by its actions in off-target organs. Several first-generation systems have already reached the market, most of these mainly based on liposomal drug formulations, such as Doxil ${ }^{\circledR}$ and DaunoXome. ${ }^{[1][2]}$ Despite extensive research efforts, the efficient clearance mechanisms of the human body for foreign materials still make site-specific delivery by nanosystems a challenge. The first hurdles new drug delivery systems are to encounter are the biological barriers, which typically result in their quick removal from the circulation before they have the possibility to even interact with the target cells. The biological barriers include uptake into Kupffer cells and macrophages in liver and spleen, facilitated by bloodstream opsonins that readily adsorb on the surface of the foreign material, and may activate the complement system $\cdot^{[3-7]}$ There is increasing knowledge on the factors that influence the recognition of the nanoparticles and their subsequent removal from the circulation. ${ }^{[8,9]}$ These include the hydrodynamic radius, morphology, surface charge and surface chemistry of the particles. ${ }^{[10-13]}$ In addition to these properties, the microenvironment of the target tissue might influence the accumulation of the nanotherapeutics. The enhanced permeability and retention (EPR) effect due to malignant growth ${ }^{[14]}$, infections ${ }^{[15]}$ or heart failure ${ }^{[16]}$ has demonstrated potential for improved extravasation of nanoparticles of optimal size to the tumor or infected and injured tissue. Several studies have shown that the optimal size for the EPR-facilitated passive targeting of nanotherapeutics is smaller than $100 \mathrm{~nm}$ but larger than $40 \mathrm{~nm} \cdot{ }^{[17-19]}$ However, recently, even nanoparticles with sizes below the effective glomerular filtration cut-off size of $10 \mathrm{~nm}$ have shown preferable accumulation to malignant tissues. ${ }^{[20]}$ This has been achieved by using $\alpha_{v} \beta_{3}$ integrin targeting cRGDY peptide ligands, which were able to prolong the tumor 
retention time and accumulation of $10 \mathrm{~nm}$ sized C-dots. ${ }^{[21]}$ Furthermore, it was reported that the bulk renal clearance was able to minimize the potential off-target toxicity by reducing the nonspecific uptake of the particles by the reticulo-endothelial system (RES) organs. In contrast to the EPR effect, there are also confining factors which may hamper accumulation of the drug delivery system to the target site. Intratumoral pressure, caused by the compromised lymphatic drainage in some aggressive tumors, has been shown to form a barrier to transcapillary transport impeding the passive targeting. ${ }^{[22]}$ After reaching the tumor tissue, the delivery system should be able to cross the plasma membrane and release its cargo into the cytoplasm. As the size of nanosystems is typically larger than permitted for passive diffusion through plasma membrane, an active transport mechanism across the membrane is needed. Clathrin-mediated endocytosis is the principal mechanism for particle internalization in mammalian cells and is facilitated by a positive surface charge of the DDS. ${ }^{[23,24]}$ This leads to the engulfment of the particles inside endosomal vesicles that efficiently fuse with cytoplasmic lysosomes. The highly degradative conditions inside the lysosomes often lead to the decomposition of the DDS and the payload upon lysosomal uptake. Therefore, there has been intensive research towards the discovery of methods enabling endosomal escape, for example those based on the proton sponge effect or flip-flop mechanism. ${ }^{[25,26]}$ The alternative is to avoid the clathrin-mediated endocytosis by modification of the DDS to utilize caveolae-mediated endocytosis instead. The caveolaemediated endocytosis results in the formation of caveosomes, which have a neutral $\mathrm{pH}$ environment and have been shown to be able to bypass fusion with the lysosomes. Functionalization of the DDSs with albumin, folic acid or cholesterol, for example, has been reported to result in uptake via the caveolae mechanism. ${ }^{[27,28]}$

Although substantial research efforts have been made towards finding methods to improve the site-specific delivery of therapeutics, it still remains an unattainable goal. In vivo imaging has an essential role in the discovery of more successful methods for improving the specificity of nanotherapeutics and for studying the effects of nanotherapeutic delivery. During the last decade, significant progress has been made in the development of methods for tracking drug delivery systems non-invasively in living systems, and in vivo imaging has proven its worth in providing valuable information on the behavior of DDSs in the living body. 
Imaging can aid in understanding the interactions between nanomaterials and biological systems. This has increased our knowledge on the mechanisms behind the recognition and removal of the particulate carriers from the circulation by the immune system. The information gained through in vivo imaging can speed up the development of new nanotherapeutic systems and hopefully will help in generating a new generation of nanotherapeutics with improved specificity. Furthermore, the integration of the diagnostic function into the therapeutic system can improve the treatment prognosis as it allows the monitoring of the drug delivery process during treatment and the individual's response to therapy (Figure 1). This approach called "nanotheranostics" is anticipated to be an important avenue towards personalized medicine and improved safety of drug therapies.

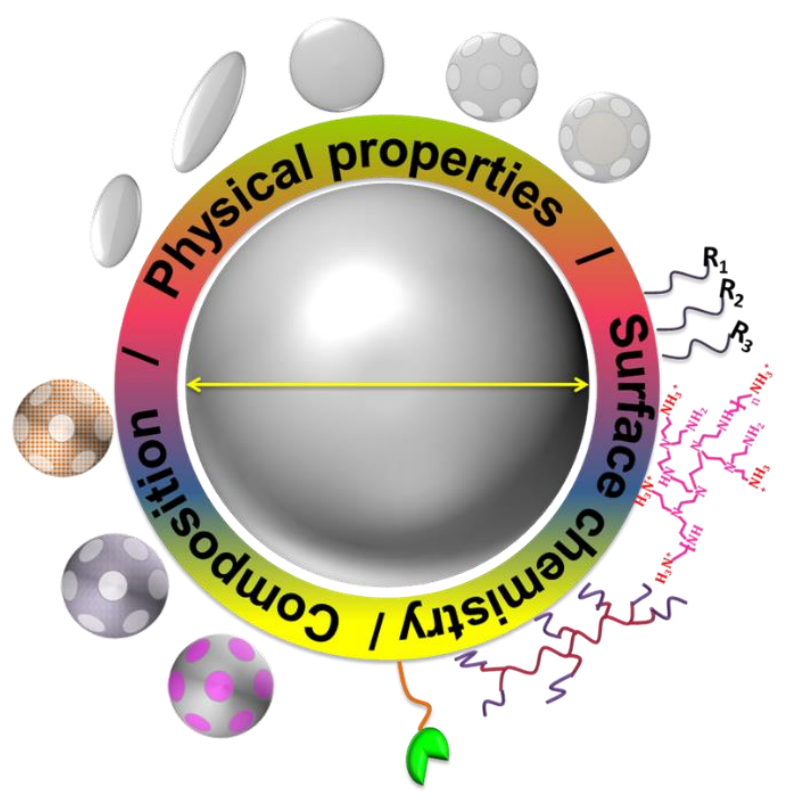

Figure 1. Design options for theranostic nanoparticles. Physical properties, surface modifications and composition of porous and non-porous nanoparticles can be tuned in order to make them powerful tools for imaging. For instance, the size and morphology of the particles can be tuned to enhance their interaction with cells and their permeability across biological barriers; reactive groups can be added on the particle surface for the attachment of different organic moieties, biomolecules or nanoscopic imaging agents; and the incorporation of MRI, optical, or nuclear imaging agents and therapeutic payloads inside the carrier structure can be realized. 


\subsection{Inorganic materials as imaging agents}

Several in vivo imaging techniques have shown to be useful for investigations in image-guided drug delivery, including magnetic resonance imaging (MRI), computed tomography (CT), positron emission tomography (PET), single photon emission computed tomography (SPECT) and optical imaging methods (OI) such as those based on luminescence or fluorescence. Typically, the nanomaterial needs to be tagged with a modalityspecific label, which allows for the tracking of the material with the imaging modality in question. In any case, it is important not to influence the biodistribution of the drug delivery system by the label in any way, for example by altering the surface charge of the system due to the surface modifications needed for enabling the labeling chemistry. ${ }^{[29]}$ Optimally, the material itself may serve as a contrast agent and no additional modifications are needed. Especially inorganic nanostructures are readily utilized as biomedical imaging probes due to their inherent detectability by a variety of different imaging modalities, exemplified by the detection of luminescent quantum dots (QDs) by optical imaging and superparamagnetic iron oxide nanoparticles (SPION) by MRI. Such novel nanoscopic MRI contrast agents are especially advantageous as scaffolds in multimodal or theranostic nanoparticulate systems, where they can be combined with optical imaging (or other modality) labels and/or the ability to deliver a drug for image-guided therapy possibilities. ${ }^{[30,31]}$ Especially nanomaterial-based probes which are optically active in the near-infra red (NIR) region can improve the sensitivity of lesion delineation over that of organic fluorescent dyes. When nanoparticles are used as carriers for molecular imaging agents, benefits similar to those associated with nanoscopic DDSs are obtained. These properties include the cellular uptake and intracellular delivery capability, the capacity to carry high amounts of different molecular compounds, the generation of a protective and stabilizing environment for the incorporated agents; and the ability to retain the cargo within the particle for extended periods of time. Utilizing this approach, sensitive fluorophores can be photostabilized and the dose of toxic imaging agents can be decreased, while the imaging time frame can be prolonged owing to the retention of the nanocarrier at the target site. Moreover, one nanoparticle can carry multiple targeting and imaging moieties, which can enhance both the sensitivity of the signal detection 
(multiple imaging agents per binding event) and the probability of the attachment to the target (multiple targeting moieties per particle). The imaging modality can be chosen depending on the nanoparticle design, whereby the combination of different nanomaterial constructs is used to create multimodal probes.

\subsection{In vivo imaging modalities}

There are different types of in vivo imaging techniques available and each of these have certain advantages and limitations in terms of sensitivity, signal attenuation and resolution. Several in vivo imaging modalities have shown potential for image-guided drug delivery; these include MRI, optical luminescence and fluorescence imaging $(\mathrm{OI})$ and nuclear imaging such as PET and SPECT. Due to their different properties, these modalities are complementary rather than competitive, and multimodality approaches are often applied in order to overcome the limitations of a single modality.

MRI is based on the spin relaxation of protons after radiofrequency pulses in the presence of a strong magnetic field. MRI can be used for the tracking of DDSs by tagging them with superparamagnetic or paramagnetic elements. These elements, such as $\mathrm{Gd}$ or Fe, can influence the signal strength of the spin relaxation of protons in the surrounding water and increase the image contrast. Superparamagnetic iron oxide (SPIO) is typically used in nanoscopic imaging agent constructs in the form of nanoparticles (SPION) loaded into a porous material if they are of ultra-small type (USPIOs), as a core material layered with other materials (core@shell structures) or inside a hollow particle. SPIONs have already been marketed under trade names such as Feridex ${ }^{\circledR}$ and Resovist ${ }^{\circledast}$, but due to their negative (black) contrast in MRI, other metal oxides such as manganese and gadolinium oxide nanoparticles with positive (white) contrast have been developed. $\mathrm{Gd}^{3+}$ or $\mathrm{Mn}^{2+}$ ions can be bound to the surface of nanomaterials by chelating groups such as tetraazacyclododecane-1,4,7,10-tetraacetic acid (DOTA), 1,4,7-triazacyclononane-triacetic acid (NOTA) or diethylenetriaminepentaacetic acid (DTPA), or they can be entrapped inside the nanomaterial by different means. Molecular chelates/complexes of $\mathrm{Gd}^{3+}$ ions are used in the clinic with trade names such as Dotarem ${ }^{\circledR}$ and Magnevist ${ }^{\circledR}$, while $\mathrm{Mn}^{2+}$ chelates/complexes are more frequently used in veterinary medicine and animal studies. The use of Gd- and Mn-based nanoparticles is still limited to research. The advantage of MRI is its 
submillimeter spatial resolution and the excellent anatomical information, which it can provide arising from the excellent contrast of the soft tissue structures. Unlike ionizing radiation, MRI is not known to cause any potentially harmful biological effects when used at low magnetic fields $(<7 \mathrm{~T})$. Furthermore, because it is based on stable isotopes, the MRI contrast agents can be added to the nanoconstructs already during their manufacturing process. A disadvantage of MRI is its relatively low sensitivity. The detection limit for Gd is approximately $10^{-5} \mathrm{~mol} / \mathrm{l}$, which hampers its use in quantification of drug delivery systems in sites with low accumulation. ${ }^{[32]}$ However, when exposed to the chemotherapeutic payload, the accumulation into these minor sites may still be a significant source of adverse effects.

In the optical imaging process, the information that can be obtained is strongly depth-weighted and will depend on the thickness and optical properties of the tissues to be imaged. ${ }^{[33]} \mathrm{A}$ suitable molecular imaging label should be without simultaneous excitation of the auto fluorescence of the tissue, and from a material point of view, the nanoscopic molecular agent carrier should not interfere with the optical imaging process. In addition, it must be sufficiently bright (high molar absorption coefficient and high fluorescence quantum yield) and have an adapted fluorescence lifetime and the largest possible Stokes shift. A large number of organic fluorescent dyes, such as fluoresceins, rhodamines, and cyanines have been developed for biological applications, but their emission regions are in the visible spectrum that matches with the auto fluorescence of the tissues. The tissues are less absorbing in the spectral window ranging from $650 \mathrm{~nm}$ to $900 \mathrm{~nm}$, and recently, most of the probes intended for optical imaging applications are designed for this near-infrared (NIR) window. While the number of available NIR fluorophores is increasing, they usually suffer from low quantum yield efficiency that limits their use. So far, the only NIR fluorophore that can be used in the clinic is Indocyanine Green (ICG). ${ }^{[33]}$ Even if the solubility of ICG is a concern, it can be improved with emulsion, ${ }^{[34]}$ liposome $\mathrm{e}^{[35]}$ and clinical grade nanoparticle-based formulations. ${ }^{[36]}$ In this respect, several advantages can be provided with the incorporation of fluorescent dyes into nanoparticles dedicated to in vivo imaging ${ }^{[37]}$ : (1) nanometer-sized materials can govern the biodistribution of the probe; (2) with the versatile design options of the nanoparticles, complex and modular tracers can be provided with different functionalities; and (3) brighter fluorescent tracers can be generated. The major disadvantage of optical imaging methods is the 
strong attenuation of the signal in tissues allowing for the whole-body imaging of small animals only and not in humans. However, intraoperative optical imaging, where the detector can be placed close to the site of the imaging probe accumulation by means of an endoscopic instrument or a hand-held device, is increasingly used in the clinic permitting signal detection also from deeper tissues. Simultaneously, the integration of optical imaging devices into commercial endoscopic instruments and surgical systems is increasing.

PET and SPECT are used in nuclear medicine for the early detection and diagnosis of cancer, and they are both utilized in nanotheranostic applications. They are noninvasive, quantitative and sensitive. Compared to other translational imaging modalities, PET has superior sensitivity. The detection threshold of PET has been shown to be 200 times more sensitive than that of MRI and 50 times more sensitive than that of fluorescencebased tomography. ${ }^{[38]}$ Sensitivity of SPECT is approximately one order of magnitude less than for PET. A disadvantage of both PET and SPECT is that often the surface chemistry of the investigated material needs to be modified with functional groups enabling stable radiolabeling of the material for in vivo studies. This should be achieved without influencing the biological properties of the DDS. Some of the important radionuclides for PET and SPECT imaging are presented in Table 1. For the incorporation of metallic radionuclides, the surface needs to be functionalized with chelating groups such as DOTA, NOTA or DTPA (i.e. the same as used for complexing paramagnetic cations for MRI). However, in some cases the nanomaterial itself may be competing with the chelating groups for the coordination of the metallic radionuclide. This is, for example, the case with surfaces with free carboxylic acid groups that are able to coordinate the cationic radiometals. Coordination to these additional sites reduces the radiolabeling efficiency of the material and may decrease the stability of the radiolabel in vivo caused by the non-optimal coordination. If not efficiently removed during the purification steps, the loosely coordinated radionuclide is easily detached in the presence of metal-containing enzymes in the blood, leading to a situation in which the observed radioactivity signal is not representative of the biodistribution of the DDS anymore and may lead to erroneous interpretation of the image. Sometimes coordination to the functional groups of the nanomaterial yields to a stable coordination. A chelator-free zirconium-89 labeling ("intrinsic radiolabeling") of mesoporous silica nanoparticles (MSNs) has been achieved by utilizing the numerous deprotonated silanol groups inside the 
pores and on the surface of the MSN. ${ }^{[39]}$ Zirconium-89 is a PET radionuclide with a relatively long half-life $\left(\mathrm{t}_{1 / 2}\right.$ $=78.4 \mathrm{~h}$ ) allowing for imaging studies within the time frame relevant for the biological interactions of nanomaterials. It is applied especially for the investigation of DDSs with long circulation time. ${ }^{89} \mathrm{Zr}$ typically exists in oxidation state +IV, in which it is a hard Lewis acid, and thus prefers hard Lewis bases as donor groups. Deprotonated silanol groups of the amorphous silica particles were shown to act as hard Lewis donors forming a stable coordination for ${ }^{89} \mathrm{Zr}^{4+}$ affording the excellent long-term stability in vivo, with only $1.5 \% \mathrm{ID} / \mathrm{g}$ of ${ }^{89} \mathrm{Zr}$ accumulating in bone at 21 days p.i. The same approach was utilized very recently for radiolabeling of MSN particles with a new investigational positron emitter titanium-45. ${ }^{[40]}$ Sometimes the chemical composition of the material allows covalent radiolabeling of the material in a position which is hydrolytically and enzymatically stable. For example, the high affinity of fluorine to silicon allows the labelling of porous silicon (PSi) by nucleophilic $\left[{ }^{18} \mathrm{~F}\right]$ fluoride. Fluorine-18 is a PET-compliant short-lived radioisotope with a half-life of $119.7 \mathrm{~min}$. The hydrolytic stability of $\mathrm{Si}^{-18} \mathrm{~F}$ bonds has been shown to be impacted strongly by the substituents adjacent to the Si atom bound to fluorine. Steric hindrance shielding the Si atom has been found to be especially important for the stability of the bond. ${ }^{[41]}{ }^{18} \mathrm{~F}$-fluorination of thermally oxidized silicon (TOPSi) leads to a $\mathrm{Si}^{18} \mathrm{~F}$ bond which is easily hydrolysed at physiological $\mathrm{pH}$. Instead, in thermally hydrocarbonized (THCPSi) surface, which is passivated against the hydrolytic attack with the sterically hindering hydrocarbon chains, ${ }^{18} \mathrm{~F}$ is covalently bound to silicon in a position with high stability against in vivo defluorination. ${ }^{[42]}$

Table 1. Some frequently used radioisotopes for PET and SPECT imaging

\begin{tabular}{lll}
\hline Isotope & Half-life & Modality \\
\hline${ }^{11} \mathrm{C}$ & $20.3 \mathrm{~min}$ & PET \\
${ }^{68} \mathrm{Ga}$ & $68.3 \mathrm{~min}$ & PET \\
${ }^{18} \mathrm{~F}$ & $119.7 \mathrm{~min}$ & PET \\
${ }^{99 \mathrm{~m} T \mathrm{Tc}}$ & $6 \mathrm{~h}$ & SPECT
\end{tabular}




\begin{tabular}{lll}
${ }^{64} \mathrm{Cu}$ & $12.7 \mathrm{~h}$ & PET \\
${ }^{123} \mathrm{I}$ & $13.2 \mathrm{~h}$ & SPECT \\
${ }^{111} \mathrm{In}$ & $67.2 \mathrm{~h}$ & SPECT \\
${ }^{89} \mathrm{Zr}$ & $78.4 \mathrm{~h}$ & PET \\
${ }^{124} \mathrm{I}$ & $4.18 \mathrm{~d}$ & PET \\
\hline
\end{tabular}

\subsection{Multimodality imaging}

In recent years, the value of combining imaging modalities has been recognised and gained popularity since the single imaging modalities currently in clinical use have either very high sensitivity and relatively poor resolution, or high resolution and relatively poor sensitivity. ${ }^{[43]}$ However, in multimodal strategies, the capabilities of the different imaging modalities are blended to prodigious effect by their in tandem usage. Instruments for the simultaneous combination of imaging technologies were pioneered in 1998 as the first PET/CT instrument that became commercially available in 2001. Along with the development of the synergistic imaging technologies, novel imaging probes have been developed to support these multimodal systems. A number of different multimodal probes have been constructed for research purposes since the single diagnostic methods are not effective enough for the accurate diagnosis of the tumour in the early stage of cancer. Meanwhile, the multimodal capability of the probes has simultaneously been devoted to the investigation of the nanoprobes' in vivo biocompatibility, biodistribution and clearance. For instance, the study of Park et al. investigated the cell labeling efficiency and biocompatibility of their already designed fluorescent silica coated magnetic nanoparticles with both in vitro and in vivo tests. ${ }^{[44]}$ In the study, mesenchymal stem cells were labeled with the multimodal imaging nanoprobe, and the labeled cells, when injected into mice, could be identified by MRI and optical imaging without the requirement for more invasive methods. In addition, they also demonstrated the biocompatibility of the multimodal nanoprobes for monitoring stem cells in vivo by employing a variety of methods to evaluate the possible changes in cell surface phenotype, cell cycle, cell proliferation, cell morphology, and apoptotic cell death. 
Biocompatibility is a prerequisite for any multimodal imaging agent for clinical applications. For silica and silicon constructs, the starting points in this regard are promising. Silica, $\mathrm{SiO}_{2}$, is permitted as a direct additive in a broad variety of foods in amounts up to $5 \%$ by weight in the EU, whereas $\mathrm{Si}$ is also naturally ubiquitous in food. Due to its favorable properties, silica has been used as a pharmaceutical excipient for decades and is classified a GRAS (generally regarded as safe) material by the FDA. The element silicon itself is an endogenous substance, and in its bioavailable form, $\mathrm{Si}$ is present as silicic acid species ${ }^{[45]}$; into which $\mathrm{Si}$ as well as amorphous $\mathrm{SiO}_{2}$ dissolve under aqueous conditions. Since only water is needed for the materials to dissolve, the term "biodegradation" is actually erroneous even though it is frequently used in describing the "biodegradation of silica/silicon". The dissolution limit of silica is $\mathrm{pH}$-dependent, and is at neutral pH about $120 \mathrm{ppm} .{ }^{[46]}$ The silicic acid species that is formed upon dissolution are renally excreted or, in the best case, even used as nutrients by the cells, ${ }^{[45]}$ as silicon is an essential component of cells throughout the human body (the silica content of human tissues ranges from 10 to $200 \mathrm{mg}$ per $100 \mathrm{~g}$ dry weight). The first studies addressing the biocompatibility of porous silicon as an implantable material date back to the turn of the century. In one of the early studies, in vivo responses to subcutaneous implantation of PSi discs were investigated by monitoring the body weight and temperature of guinea pigs for 26 weeks. The results were combined with histopathological analysis of the tissue in the vicinity of the implant. ${ }^{[47]}$ Porous silicon was shown to exhibit tissue compatibility comparable to that of pure titanium, a material widely used for surgical applications. The results were supported soon by Rosengren et al., who reported the comparison of the cellular reactions induced by the implantation of porous and planar silicon and titanium implants in rat abdominal wall. ${ }^{[48]}$ However, when it comes to nanoconstructs, the material composition and tissue compatibility of the bulk material are obviously far from being the only decisive factors for the safety of the nanomaterial construct. Analogous to other biological effects, the biocompatibility and toxicity will depend on the material attributes such as particle size, shape, charge, surface chemistry, porosity, surface area and so forth. In the specific case of silica, also synthesis aspects such as the thermal history which affects the rate of dissolution along with the aforementioned parameters, plays a role. ${ }^{[4]}$ Additionally, the route of administration, dose, and exposure time will have a tremendous effect on the outcome of the toxicity studies. 
Different cells and tissues are further unequally susceptible to particle exposure, which, along with the great number of different assays used and lack of standardization across studies trying to address these issues, make drawing any general conclusions extremely difficult. ${ }^{[50]}$ The interested reader is referred to reviews that have regardless of these challenges made an attempt to analyze the accumulated studies with regard to specific particle types, e.g. MSNs. ${ }^{[51]}$ PSi micro- and nanoparticles have demonstrated favorable biocompatibility with complete degradation to non-toxic silicic acid in 4 weeks after parenteral or intravitreal administration when using untreated particles without any stabilizing surface modifications. Histopathological analysis of the tissue sections for possible toxic and inflammatory responses from the organs with the highest exposure (the kidneys, liver, spleen, and eye in the case of intravitreous administration) has not revealed any measurable in vivo toxicity during observation periods of 1 to 12 months. ${ }^{[52,53]}$ However, stabilization of the PSi surface with thermal oxidation, alkylation or aminosilanization significantly affects the degradation kinetics in vivo and can also contribute to the in vivo biocompatibility of the material. ${ }^{[52,54]}$ Thermal oxidation has been shown to lengthen the degradation half-life of PSi microparticles in the eye up to 2 months and thermal hydrosilylation up to 4 months, without any increased toxicity. In the heart, thermally hydrocarbonized and thermally oxidized PSi micro- and nanoparticles exhibited good in vivo biocompatibility and were found non-toxic after their administration via a local myocardial injection. ${ }^{[55]}$ However, the thermally hydrocarbonized PSi microparticles (with sizes 7 and $17 \mu \mathrm{m}$ ) caused release of inflammatory cytokines and activation of fibrosis-promoting genes within a week of particle administration. Although biocompatibility of porous silicon has been demonstrated in several studies, it may be compromised by unfavorable size, shape or surface chemistry of the particles. In the absence of standardized test protocols for the evaluation of nanotoxicity, the acceptance of the silica-based "C-dots" for human Phase I clinical trials in 2010 must be regarded as a highly positive development. ${ }^{[20]}$ Many other research efforts have also focused on successful translation of nanoprobes from bench to bedside; however, it is noteworthy that these multimodal C-dots combining optical imaging (OI) with positron emission tomography (PET) capability, are the only radiolabeled multimodal nanoparticle formulation that has found its way into clinical trials so far. ${ }^{[20]}$ 


\section{In vivo imaging of silica based materials}

\subsection{Preparation of amorphous silica based nanoparticles}

Amorphous silica itself in bulk or in the nanosized form does not possess any features enabling use as an imaging probe due to its optical transparency. However, silica has attractive characteristics to be exploited in various imaging probe designs. In practice, a variety of methods can be used for the production of silica nanoparticles. Silica nanoparticles are a type of colloidal amorphous metal oxide, which can be synthesized by a bottom-up approach e.g. via the sol-gel method. In the sol-gel system, the formation of oxide network through polycondensation reactions of molecular precursor in liquid occurs, i.e. monomeric silicon alkoxide precursors are hydrolyzed and subsequently condensate owing to the added acid or base catalyst. Either Stöber or reverse microemulsion methods are usually applied for the preparation of non-porous silica nanoparticles. The Stöber method is one of the most widely used synthetic approaches to prepare nonporous silica materials. The process was discovered in 1968 by Werner Stöber et al. ${ }^{[56]}$ and built on earlier work of by G. Kolbe in 1956. ${ }^{[57]}$ In the study of Kolbe, synthesis of monodispersed silica particles based on the hydrolysis and subsequent condensation of silicon alkoxides in ethanol was investigated. Later on, Stöber et al. systematically worked on the experimental conditions of this reaction, and investigated the controlled growth of spherical silica particles, which is now the well-known Stöber process. Subsequently, numerous synthesis routes and various precursors have been used for the preparation of silica nanoparticles with different sizes and shapes.

The reverse microemulsion method is another approach that is used for the production of non-porous silica nanoparticles. In this process, spherical silica particles might be conveniently synthesized by supplementing silicon alkoxide, typically tetraethylorthosilicate (TEOS), to a reverse water-in-oil microemulsion. During the synthesis, the silica nanoparticles can be grown inside the microcavities by carefully controlling the addition of silicon alkoxides and catalyst into the medium containing reverse micelles. ${ }^{[58]}$ The production of silica nanoparticles is achieved by the diffusion of the alkoxide into the water droplets, followed by hydrolysis of 
the alkoxide and formation of oxy-hydroxy-silicate species and alcohol, which is a so-called a microemulsion process (Figure 2). ${ }^{[59]}$

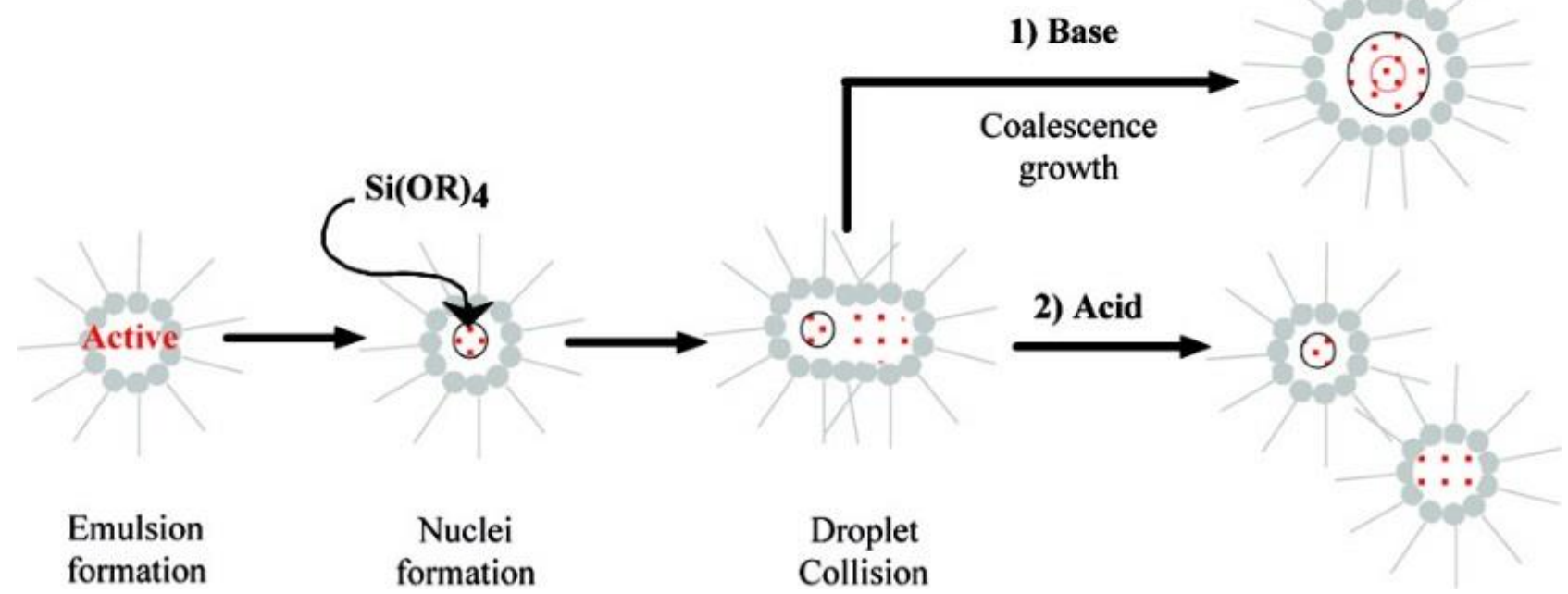

Figure 2. The microemulsion synthesis of silica particles both under basic and acidic conditions. Reprinted with permission from ref. 59. Copyrights 2007 American Chemical Society.

The preparation of mesoporous silica materials was first reported by Yanagisawa et al. in $1990^{[60],[61]}$ using a different synthesis approach than the more well-known studies by Mobil Oil scientists from 1992, where the synthesis of silica/alumina ordered mesoporous molecular sieves designated as M41S was reported. ${ }^{\left[{ }^{[2]}\right.}$ The preparation of these mesostructured silicates are based on self-assembly principles in concentrated surfactant $\left(C_{n} T M A\right)$ aqueous solution and their interactions with monomeric silicates (Figure 3 ). For the actual particle growth, the synthesis of mesoporous particles on the nanoscale, i.e. mesoporous silica nanoparticles (MSNs) can employ the same principles as the Stöber process. 


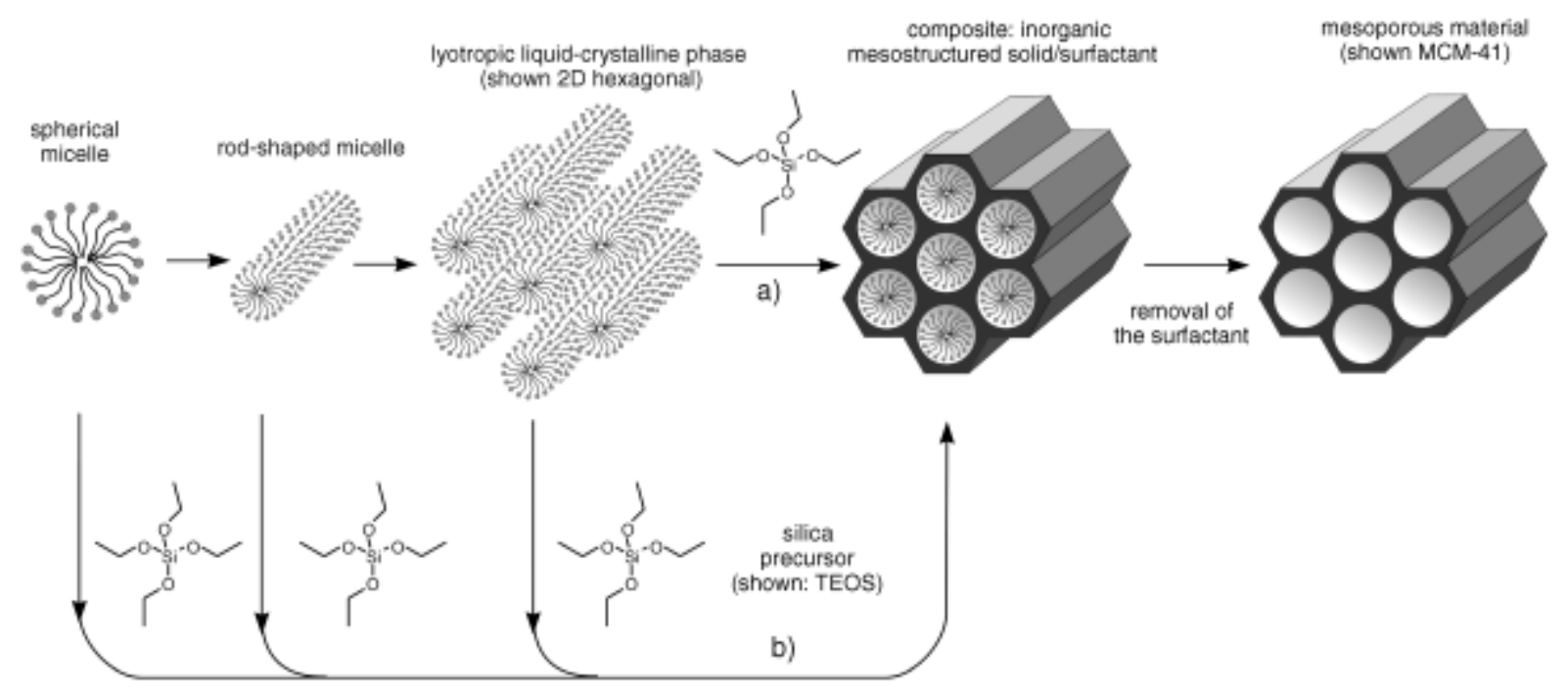

Figure 3. Formation of mesoporous silica materials by structure-directing agents: a) true liquid-crystal template mechanism, b) cooperative liquid-crystal template mechanism. Reprinted with permission from ref. 62. Copyrights 2006 Wiley-VCH Verlag GmbH \& Co.

Flexible design options are provided in the production of both non-porous and porous silica nanoparticles. They can have well-defined and tunable physicochemical properties, the surface chemistry can be altered by functionalization with different organic surface groups or polymeric coatings, to which a vast array of different biomolecules and/or molecular or other nanoscopic imaging agents can be conjugated. ${ }^{[63]}$ Furthermore, the porous structure of MSN or even hollow MSNs provides cavities that can host a large amount of various small-molecular compounds, biomolecules, organic or inorganic imaging agents. The incorporation of imaging agents into the silica matrix can also be provided by the construction of layer-bylayer structures to develop hybrid materials incorporating different kinds of nanoparticles. ${ }^{[64]}$ Additionally, the extent of silanol groups on their surfaces makes them hydrophilic, which is a prerequisite for any in vivo application.

\subsection{Silica as a carrier material for molecular imaging agents}

Silica as an inorganic (ceramic) material can provide efficient protection for the encapsulated active molecules from the environment ${ }^{[65]}$ (e.g. metabolic enzymes, harsh $\mathrm{pH}$ and elevated temperatures) and 
especially mesoporous silica materials can carry up to their own weight of cargo molecules. ${ }^{[66]}$ Consequently, MSNs are most recognized for their potential in drug delivery, but as mentioned above, the same benefits experienced by drug molecules in these delivery systems can be applied in the design of nanoscopic imaging probes by encapsulation of molecular imaging agents. The dye molecules can be incorporated into silica matrices either by in situ or post-synthesis methods. The physical adsorption of dye onto the surface of readymade silica particles is another option, but this poses the risk of leakage and migration of the dye during application, unless the pores are further capped e.g. by a surface coating. In the literature, different dye doping strategies into silica matrices have been compared to evaluate the homogeneity and the optical properties of particles after doping. ${ }^{[67]}$ In the extensive study of Börgardts et al. they have investigated the effects of in situ co-condensation and post-synthesis grafting strategies for the homogeneity of incorporated Nile Red (NR) dye in the silica matrices, and the consequential optical properties of the resulting silica hybrid material. Their results revealed that the post-synthesis approach retains the initial pore structure of the silica. However, a non-uniform distribution of NR molecules is obtained because of the preferential accumulation of the dye molecules at the pore openings. On the contrary, the in situ co-condensation approach yielded a homogeneous distribution of the NR throughout the silica matrix. However, in the in situ co-condensation approaches, an optimal dye doping level is a crucial factor to be considered, since the organic dye molecule become a component of the matrix, and thus the increment in the organic functionalization of the silica matrix may lead to the collapse of the structural order of the silica. The dependence of the quantum yield on the dye incorporation strategy was also investigated. The maximum quantum yield of the material prepared via the in situ co-condensation route was found to be $4 \%$ less than for the post-grafted one. In addition, at equivalently high concentrations of dye in the matrices, the in situ preparation yielded a higher degree of fluorescence quenching as compared to post-grafted preparations.

As frequently shown and reviewed in the literature, the inherent chemical, thermal and dimensional stability of silica matrices is advantageous for their use as molecular imaging agent carriers. However, it is important to be aware of and carry out systematic investigation of the characteristics of the obtained materials that could affect the materials' properties as imaging probes. The homogeneous introduction of organic groups 
into the silica framework that can endow an imaging process with improved imaging properties, and chemically stabilized imaging agents is a crucial property to this end. ${ }^{[68]}$

\subsection{Silica as a construct in core@shell materials}

Silica is also the most widely used coating material for other inorganic nanoparticles. The use of silica as a coating material is mainly due to the thus provided stability to the core material, easy regulation of the coating process, chemical inertness, controlled porosity, facile processing, and optical transparency. Nonporous silica coatings are also utilized in different imaging modalities for instance in photoacoustic imaging for increasing the photothermal stability of the core material (either $\mathrm{Au}^{[69]}$ or SPION ${ }^{[70]}$ ). Furthermore, it is frequently used as a biocompatible coating on for example SPIONs simultaneously allowing for facile further functionalization and protection of the core material against $\mathrm{pH}$ changes in the environment, which otherwise can lead to e.g. oxidation or even dissolution of the core material. ${ }^{[71]}$ Silica coating of colloidal nanoparticles can be carried out in water-in-oil (W/O) microemulsions, via the Stöber synthesis method or by use of silane coupling agents and sodium silicate water-glass methodology. ${ }^{[72]}$ In such sandwich structures, or core@shell materials, the two (or more) materials account for their own distinct functions, and/or a third material can be used to physically separate the other two materials that would otherwise lead to passivation of the activity of one of the materials, such as luminescence quenching. ${ }^{[73,74]}$ Especially in the design of twoin-one fluorescent-magnetic nanocomposites, the risk of quenching of the fluorophore by the magnetic core is imminent, since the magnetite strongly adsorbs the transmitted light. ${ }^{[75]}$ Here, a silica coating can be utilized as an effective barrier between the magnetic core and the fluorophore, which may further be controlled by the thickness of the silica shell (Figure $4 A-C) .{ }^{[76]}$ Such a composite is multimodal not only from an in vivo imaging point of view, but can also be dually detected in fluorescence-assisted cell sorting in both fluorescence and reflection modes (Figure 4F). Furthermore, the magnetic core can be useful for not only MR imaging (Figure 4D,E) but can also be exploited for magnetic targeting, magnetofection or magneticallyenhanced cellular labeling (Figure 4B,D-E). If the cores are coated with mesoporous silica, the porous shell can also be utilized for incorporation of active molecules such as drugs or molecular imaging agents and/or 
for more efficient surface functionalization that attainable on a non-porous surface (Figure 4B). The most typical thus formed, so-called core@shell construct, is composed of an iron oxide core coated with a mesoporous silica shell, where the core is intended for MR-imaging and pores are intended for drug loading. Even though originally developed for hydrophobic iron oxide cores, ${ }^{[77]}$ the synthesis protocols have later been generalized to be applicable also for hydrophilic material cores, ${ }^{i}$ whereby porous core@shell designs based on also manganese oxide ${ }^{[78,79]}$ (magnetic activity) as well as lanthanide-doped inorganic nanocrystals $\left[\mathrm{Gd}_{2} \mathrm{O}_{3}: \mathrm{Eu}^{3+}\right]^{[80]}$ and nanodiamonds ${ }^{[81]}$ (optical activity) have been constructed for similar purposes. The todate developed coating strategies are generic in that the core material can easily be substituted for other materials, including platinum, ${ }^{[82,83]}$ gold, ${ }^{\left[{ }^{[8-88]}\right.} \mathrm{NaCl}$ crystals ${ }^{[89]}$, quantum dots, ${ }^{[7]}$ UCNPs (upconverting nanophosphors) ${ }^{[90]}$ and silver. ${ }^{[91]}$
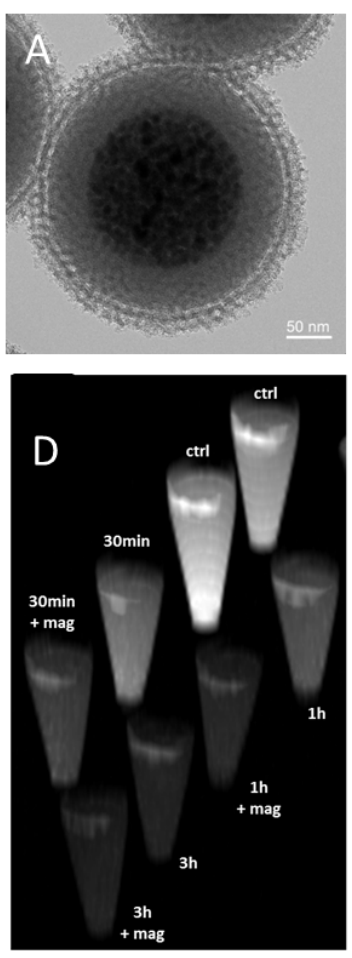

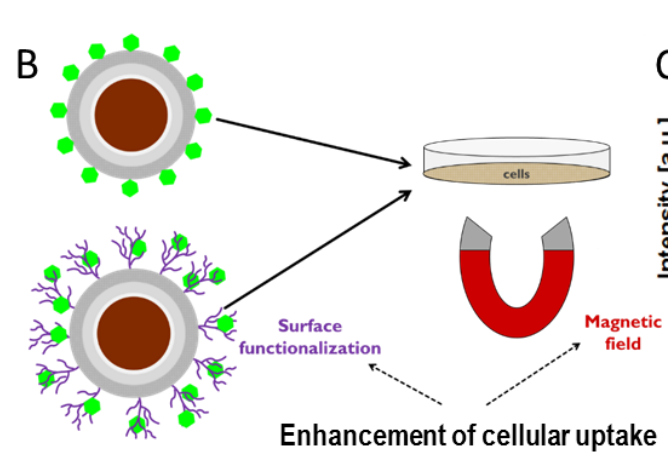

$\mathrm{E}$

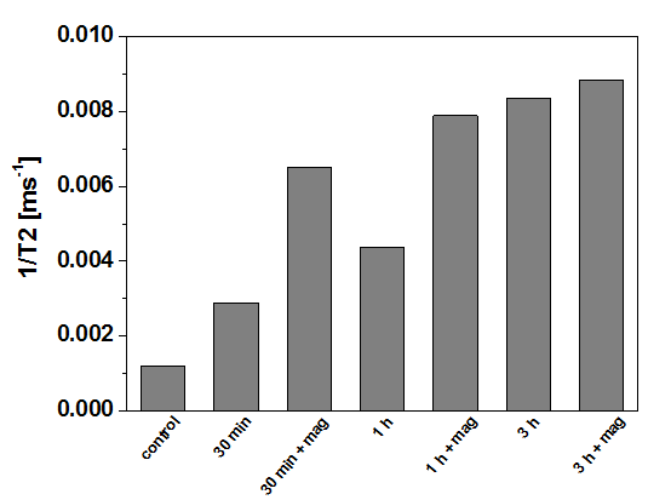

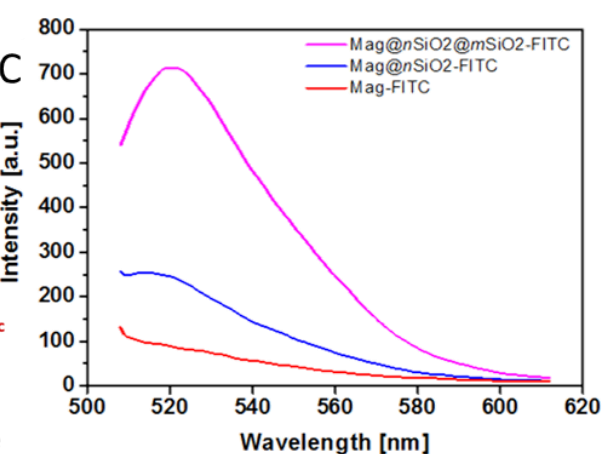

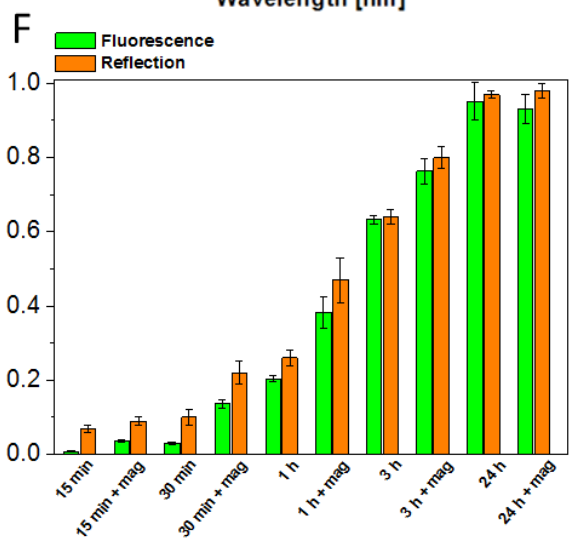

Figure 4. Core@shell@shell ( $\left.\mathrm{Mag} @ n \mathrm{SiO}_{2} @ m \mathrm{miO}_{2}\right)$ constructs consisting of a superparamagnetic iron oxide core (Mag), a non-porous silica inner shell $\left(n \mathrm{SiO}_{2}\right)$ separating the core from the mesoporous silica outer shell $\left(m \mathrm{SiO}_{2}\right)$. A) TEM image showing the morphology and structure of the magnetic cores coated with non-porous and porous silica layers; B) The effect of surface functionalization and an external magnetic field on cellular 
labeling of $\mathrm{Mag@nSiO}{ }_{2} @ m \mathrm{miO}_{2}$ particles was studied, whereby the cellular uptake of the surface functionalized particles could readily be further enhanced by an applied magnetic field; C) Fluorescence intensities, of similarly FITC-labeled magnetic core particles or magnetic core@shell particles with increasing number of silica layers, were measured in HEPES buffer (pH 7.2) between 510 and $610 \mathrm{~nm}$ using $488 \mathrm{~nm}$ excitation. The importance of the thicker non-porous silica barrier (towards the magnetite core) for the fluorescence intensity is demonstrated; D) MRI comparison of labeled cells as a function of treatment time with and without the presence of an external magnetic field; E) The $1 / T_{2}$ values, derived from the corresponding images, as a function of various labeling time points. The trend, which these results show, confirm the positive effect of the magnetic field on the cellular uptake. When a magnetic field was applied for $30 \mathrm{~min}$, an almost 6 -fold increase in $1 / T_{2}$ was found compared to the control cells; F) Cellular uptake of PEI-surface functionalized $\mathrm{Mag} @ n \mathrm{SiO}_{2} @ m \mathrm{miO}_{2}$ particles as a function of time, determined by flow cytometry both based on fluorescence and reflection. A magnetic field can readily enhance the uptake of the particles. The magnetically enhancement is more pronounced for short time intervals, but even after $3 \mathrm{~h}$ of incubation a clear difference is seen. This simultaneously demonstrates that results obtained from fluorescence and reflection based measurements are comparable if no particle aggregation occur, which in this case was avoided by appropriate surface functionalization with PEI. Adapted with permission from ref. 76. Copyrights 2014 The Royal Society of Chemistry.

\subsection{Silica nanoparticles in optical imaging}

In the design of optical imaging probes, the silica matrix provides improved mechanical strength and chemical stability to the loaded molecular imaging agent, protects it against enzymatic degradation, resist photobleaching and supply a constant environment in chemical terms. ${ }^{[22]}$ [93] In addition, the photophysical inertness of silica, its transparency under visible light and dullness in energy- and electron-transfer processes that may quench the fluorescence/photoluminescence of the dyes linked to the matrix, makes the material a promising candidate for the design of imaging probes. ${ }^{[94]}$ 
In the beginning of the 90s, the incorporation of organic fluorescent dyes into non-porous silica matrices was generally carried out by using the Stöber synthesis method. ${ }^{[95]}$ Here, classification distinction has been made between systems presenting the signaling units on the surface (dye coated silica nanoparticles, DCSNs) and with systems entrapping the dyes inside the silica matrix (dye-doped silica nanoparticles, DDSNs). ${ }^{[96]}$ Subsequently, many attempts were also devoted to incorporate fluorescent dyes into porous silica matrices to serve as a carrier for the dyes. In the specific case of fluorophores, the incorporation into such a ceramic matrix could intuitively lead to an enhanced photostability and quantum yield of the fluorophores. ${ }^{[97]}$ These effects can be caused by the stabilizing, non-reactive matrix that prevents reactions with excited molecules and by disallowing dynamic quenching, besides providing a protective environment from the surroundings. On the other side, many fluorescent silica nanoparticles are prepared by physical encapsulation of the dyes, which may leach from the matrix under physiological conditions. In order to eliminate such leakage, covalent conjugation methods of the dyes into the silica matrix or on the surface of silica nanoparticles have been attempted. For instance, in the study of Mader et al. ${ }^{[98]}$ surface modified silica nanoparticles were linked by means of azide-alkyne click chemistry and in the study by Sen Karaman et al. ${ }^{[99]}$ a post-synthesis grafting approach was carried out onto silica nanoparticles with a pre-prepared fluorescein isothiocyanate (FITC)modified aminopropyltriethoxysilane solution. Another approach is the incorporation of already dye conjugated silane precursors into the synthesis solution, which is also frequently used in order to covalently link dye molecules into the silica matrix with a homogeneous distribution and high stability. ${ }^{[100],[101],[102]}$

On the non-porous silica NP side, luminescent dye-doped silica nanoparticles (so-called "FloDots") have been developed more than a decade ago, and utilized for ultrasensitive bioanalysis and diagnosis. ${ }^{[103]}$ Other similar constructs include organically modified silica (ORMOSIL) ${ }^{[104],[105]}$ and Cornell Dots (C-dots) ${ }^{[106]}$, the latter of which has even entered Investigational New Drug (IND) human clinical trials in 2010 for cancer diagnosis. Here, 6-7 $\mathrm{nm}$ sized silica-inorganic hybrid C-dot nanoparticles have been administered to patients with metastatic melanoma. ${ }^{[107]}$ Non-porous luminescent silica nanoparticles are quite established as tools in 
different in vitro imaging, diagnostic and sensing applications, ${ }^{[108]}$ likely due to their optical transparency and photophysical inertness, enhanced resistance to photobleaching, provided mechanical strength and chemical stability for the loaded species and almost constant environment in chemical terms and biocompatibility as compared to e.g. Quantum Dots. ${ }^{[109]}$ In the study of Brites et al, aminopropyl ethoxysilane (APTES) incorporated metal-free luminescent silica nanoparticles were prepared and the light-emission features of silica nanoparticles have been pointed out through time-resolved spectroscopy. The emission process is found to be governed by donor-acceptor mechanisms mediated by the $\mathrm{NH}_{2}$ groups of APTES and $\bullet O-O-$ $\mathrm{Si} \equiv\left(\mathrm{CO}_{2}\right)$ oxygen-related defects in the silica network, as detected by electron paramagnetic resonance in diureasil hybrids. ${ }^{[110]}$

The major drawback with non-porous fluorescent dye-doped silica nanoparticles is probably the dye-rich core. The close proximity is prone to lead to aggregation-induced fluorescence quenching via some nonradiative pathway, such as molecule-molecule interaction, electron transfer, or isomerization within the solid matrix. However, the same phenomena can also be utilized to induce enhanced fluorescence by the correct choice of dyes to achieve aggregation-enhanced fluorescence (AEF). ${ }^{[111]}$ Similarly, co-encapsulation of a suitable Förster/fluorescence resonance energy transfer (FRET) dye-pair allows for the fluorescence to be activated by two-photon excitation, again taking advantage of the close proximity of the incorporated dyes.

When porous matrixes are used, this quenching issue is more easily circumvented. Within the nanometersized pores of mesoporous silica nanoparticles, the fluorescent dye molecules can be homogeneously distributed and, consequently, well-separated from each other. This arrangement limits the disadvantages associated with many other nanoparticle structures where the dyes are closely packed, e.g. in the core of the particle, leading to fluorescence quenching. ${ }^{[112]}$ Sokolov et al. prepared "ultrabright fluorescent mesoporous silica nanoparticles" ${ }^{[113-115]}$ by physically entrapping fluorescent dyes within surfactant-filled MSNs, in this case though limiting their usage within biological systems since the cationic surfactant templates $\left(C_{n} T A B\right)$ used are highly cytotoxic. However, enhanced photostability over free dye was corroborated and, the relative 
brightness per particle was further claimed to be up to forty times of similarly sized quantum dots. ${ }^{[115]}$ Similar advantages have also been obtained with surfactant-free MSNs both in vitro and in vivo ${ }^{[100]}$, both for covalently conjugated as well as physically entrapped (adsorbed) fluorophores, emphasizing the role of the stabilizing silica matrix. In general, significantly higher loading degrees of dye can be obtained by adsorption strategies as compared to covalent conjugation; but in this case, the maximum dye loading degree before fluorescence quenching starts to occur should be determined. For indocyanine dyes (Dil and DiO) a dye loading of approximately $1 \mathrm{wt} \%$ provided the maximum fluorescent intensity. ${ }^{[116]}$ In this specific case, the MSNs were used as sustained-release containers for the fluorescent dyes which provided a stable fluorescent signal of labelled tumour cells for one month in vivo (see section 2.5).

As mentioned before, silica itself is optically transparent contrary to many other inorganic materials; Furthermore, in what was initially believed to be label-free luminescent organosilica nanoparticles, where the luminescence was thought to stem from defects in the silica matrix, the luminescence was later showed to be originating from carbon dots formed in the calcination process. ${ }^{[117]}$ Specifically, as constructs in optical probes, the silica shell should not interfere with the core signal due to its transparency. On the contrary, the presence of a silica shell can even enhance the signal if the core is prone to be quenched by water, as is the case with UCNPs. ${ }^{[18]}$ For the same reason, silica shells can also be used to coat organic nanoparticles doped with water-sensitive dyes such as rare-earth metal (lanthanide) complexes. ${ }^{[119]}$ When mesoporous silica is used as a carrier for such molecules, also this construct needs to be coated in order to protect the core (MSN + lanthanide complexes) from water. ${ }^{[120]}$ In fact, also the C-dots have been regarded core@shell structures, where the core is the dye-rich center formed by co-conjugation of fluorophores and organosilanes, which has subsequently been coated with an all-silica thin shell for protection. A slightly enhanced signal has also been observed for nanodiamonds (ND), even though they are not water-sensitive in themselves. ${ }^{[81]}$ Here, NDs have been recognized as a potential alternative to QDs as they are non-cytotoxic, have excellent mechanical properties and display bright and stable fluorescence. However, their morphology is typically quite irregular, their particle size distribution rather wide, and they have a large variability of undefined 
surface groups. This may cause problems in terms of aqueous dispersibility, controlled further surface functionalization and predictable biobehavior, which can be overcome by creating core@shell structures comprising an ND core with porous or non-porous silica shells. ${ }^{[121]}$ In the case of a porous shell, this not only increases the degree of active agents that can be carried by the nanoscopic system but also enhances the dispersibility in biological media and creates a homogeneous and easily modifiable particle surface. Furthermore, ND of different origin is usually purified in strong acids after production, which results in a range of different functional groups being created on the surface of the ND due to the oxidation of residual carbon. Consequently, the ND surface charges range from highly positive to highly negative, and no "characteristic" surface charge exists. The majority of surface groups on NDs with negative charges are carboxylic acid groups resulting from oxidation, which can be utilized to promote the self-assembly of cationic CTAB micelles directly onto the ND surface. Added silica species can subsequently co-assemble with the CTAB micelles, and condense to form a silica mesophase. When coatings on nano-scaled cores are considered, ethanol is a paramount addition. ${ }^{[122,123]}$ The thickness of the resulting porous coatings can be tuned down to a couple of tens of nanometers by adjusting the water/ethanol ratio in the synthesis, thus enabling rational size control of the resulting composite particles. ${ }^{[81]}$

\subsection{Optical in vivo imaging of silica nanomaterials}

Due to depth limitations and tissue autofluorescence, optical in vivo imaging is largely limited to animal studies. In animal imaging, efforts have been laid on creating targeted optical probes for diagnostic purposes or for cellular imaging and tracking. Rosenholm et al. used MSNs as sustained-release carriers for commercially available fluorophores, yielding self-regenerating cell labels for long-term cellular tracking (Figure 5). ${ }^{[116]}$ Covalently conjugated (hydrophilic) fluorophores were compared to physisorbed (hydrophobic) fluorophores, whereby the hydrophilic fluorophores yielded much brighter imaging probes as such, but when used for cellular labelling, the hydrophobic fluorophores provided enhanced retention of the fluorescent signal inside the cells. In vivo, the fluorescent signal could be detected for a period of 1 month

(Figure 5A). Moreover, ex vivo analysis revealed fluorescently labelled metastatic colonies in lymph node and 
rib (Figure 5B and C), which highlighted the capability of the developed probes for metastasis tracking. Furthermore, when the same was repeated in the chorioallantoic membrane (CAM) model, circulating tumour cells were clearly detectable real-time in the vasculature.

Lee and co-workers prepared NIR dye, ICG encapsulated MSNs, showing that the encapsulation of ICG molecules into the MSN matrix is competent as a contrast agent with high efficiency for optical imaging of tissue in-depth. ${ }^{[124]}$ The successful use of fluorescence imaging on small animal models also encourages the development of clinically applicable techniques for the diagnosis and treatment of arthritis. ${ }^{[125]}$ It has also been reported that optical imaging can be as a tool for early detection and therapeutic monitoring of joint inflammation rheumatoid arthritis. A recently developed folate-targeted near-infrared fluorescence probe (NIR2-folate) has been tested for in vivo imaging of arthritis in in vivo models of mice. ${ }^{[126]}$

In conclusion, the reported studies of cellular labelling and tracking and the biodistribution of dyeincorporated MSNs indicate that MSNs are viable alternatives to costly commercial optical probes and address a range of shortcomings for existing cellular labelling probes with respect to the feasibility to freely choosing dye cargo from commercially available dyes, high cellular labelling efficiency and retention, lack of cytotoxicity, preserved fluorescence intensity signal retention in vivo, and the possibility of real-time detection of circulating metastatic cells and stem cells. ${ }^{[116]}$

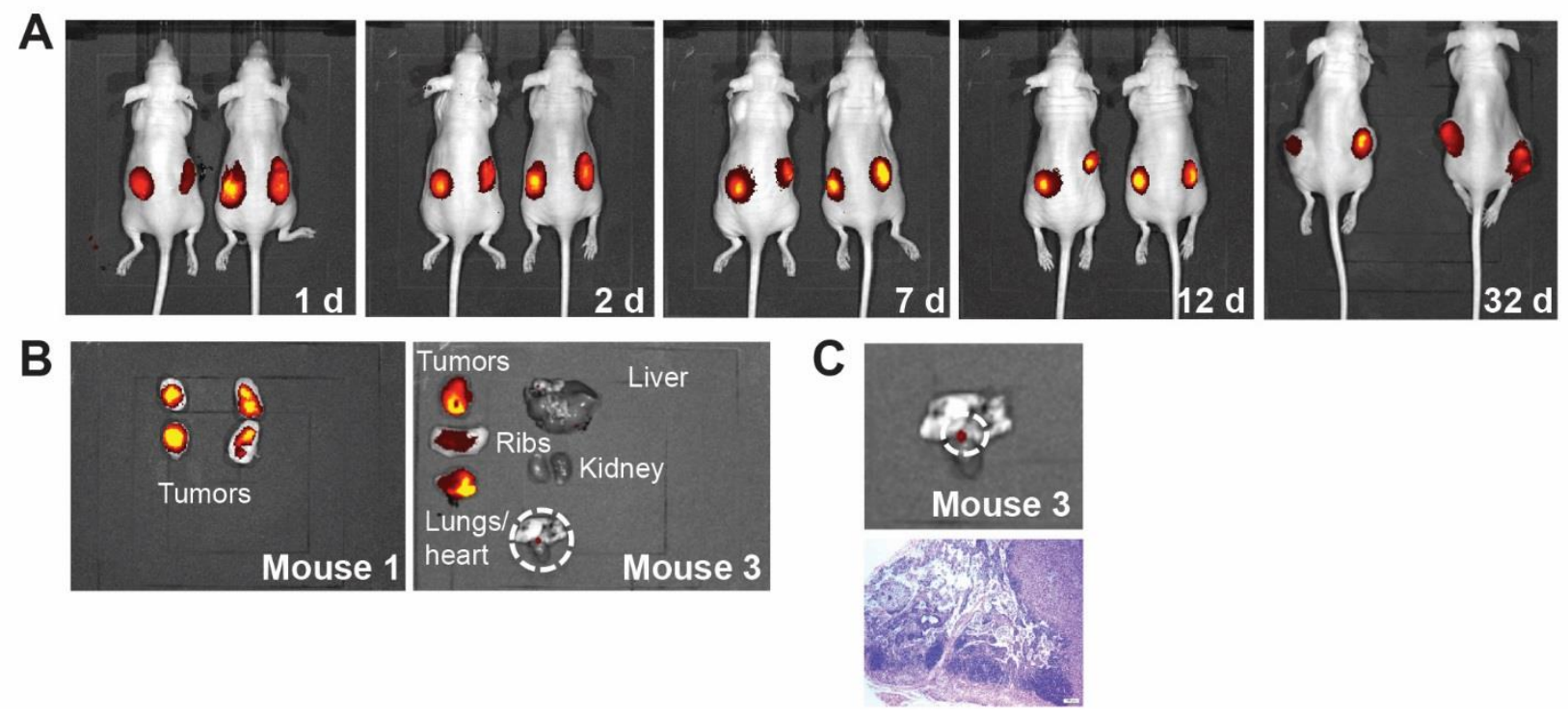


Figure 5. In vivo imaging of fluorophore-loaded MSN labeled MDA-MB-231 breast cancer cells xenografted in mice. A) The cells were visible for a period of 32 days. B, C) Ex vivo imaging of isolated organs verified tumor labeling and allowed for the detection of metastatic colonies. Reprinted with permission from ref. 116. Copyrights 2016 John Wiley and Sons.

However, because of the depth limitation optical imaging is still of minor importance for human imaging. In 2010, the C-dots i.e. a dye-encapsulated silica nanoparticle of approximately 7-nm diameter, was approved for a first-in-human clinical trial for use as a cancer diagnostic probe. These C-dots were tagged with cyclic arginine-glycine-aspartic acid peptide targeting ligands and radioiodine for PET imaging, and were shown to exhibit high affinity/avidity binding, favourable tumor-to-blood residence time ratios, and enhanced tumorselective accumulation in $\alpha v \beta 3$ integrin-expressing melanoma xenografts in mice; and owing to their ultrasmall size, they can be renally excreted. ${ }^{[127]}$ The human studies found translatable results, indicating a well-tolerated inorganic particle tracer exhibiting in vivo stability and distinct, reproducible pharmacokinetic signatures defined by renal excretion; consequently suggesting safe use of these particles in human cancer diagnostics.

\subsection{Cherenkov luminescence imaging with mesoporous silica nanoparticles}

Cherenkov luminescence $(\mathrm{CL})$, exemplified by the characteristic blue glow of the cooling water in a nuclear fission reactor, occurs when energetic, charged particles travel at a velocity equal to or greater than the phase velocity of light in the surrounding medium. ${ }^{[128,129]}$ Since $C L$ also occurs in tissues with several common $\beta$-emitting radionuclides (both positron and $\beta^{-}$) and $\alpha$-emitters in clinical use ${ }^{[130]}$, the biomedical imaging applications of $\mathrm{CL}$ have attained increased interest over the past decade. Additionally, $\mathrm{CL}$ imaging has been reported with a number of clinical radiotracers, including $\left[{ }^{18} \mathrm{~F}\right] \mathrm{FDG}{ }^{[131]}$, and therapeutic radioisotopes, such as ${ }^{90} \mathrm{Y}^{[132]}$ and ${ }^{225} \mathrm{Ac}{ }^{[133]}$, that themselves are not amenable to nuclear imaging. Especially in preclinical research, CL allows the use of less costly, high-throughput optical imaging systems for the visualization of the radioactivity biodistribution, and with associated techniques like Secondary Cherenkov Induced Fluorescence 
Imaging (SCIFI) ${ }^{[134]}$ and Cherenkov Radiation Energy Transfer $(\mathrm{CRET})^{[135]}$, the activatable optical detection of nuclear imaging probes. Both SCIFI and CRET rely on the use of fluorochrome-bearing nanomaterials to convert the energy absorbed from the Cherenkov radiation to fluorescence, with greatly increased depth of detection in tissues. ${ }^{[136]}$ Although mesoporous silica and silicon as materials are both suitable for the development of fluorochrome-bearing nanovectors for $\mathrm{CL}$ imaging, examples in literature remain scarce to date, possibly as the number of regulatory body -approved silica and silicon materials for clinical use is smaller than for other types of nanomaterials. Recently, however, Kamkaew and co-workers, reported the successful use of hollow mesoporous silica nanoparticles (HMSNs) utilizing Cherenkov radiation generated by the positron emitter ${ }^{89} \mathrm{Zr}$ for dual $\mathrm{CL}$ imaging and photodynamic therapy (PDT) by the excitation of the photosensitizer Chlorin e6 (Ce6) in a mouse model of mammary adenocarcinoma (Figure 6). ${ }^{[137]}$ For other nanomaterials in $\mathrm{CL}$ imaging, the reader is referred to the recent, comprehensive review on the topic by Shaffer and co-workers. ${ }^{[138]}$

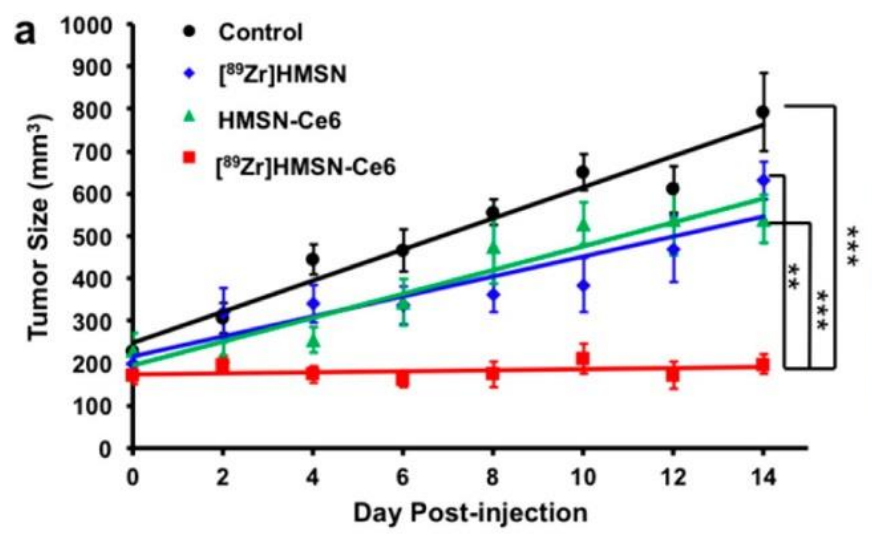

b

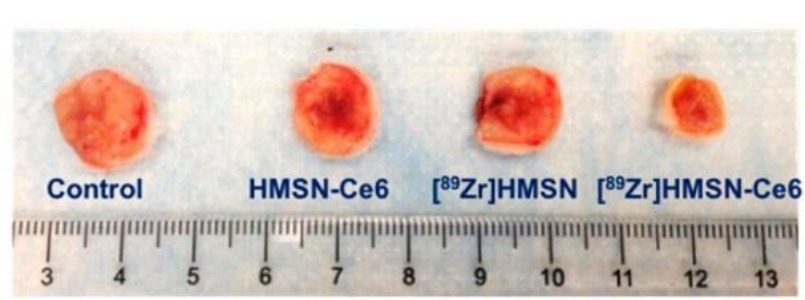

C
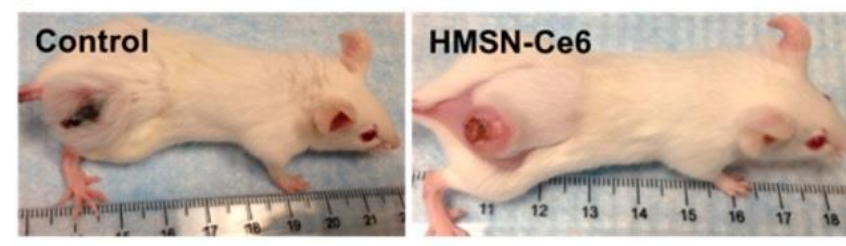

[89 Zr]HMSN

$\left[{ }^{89} \mathrm{Zr}\right] \mathrm{HMSN}-\mathrm{Ce} 6$

d
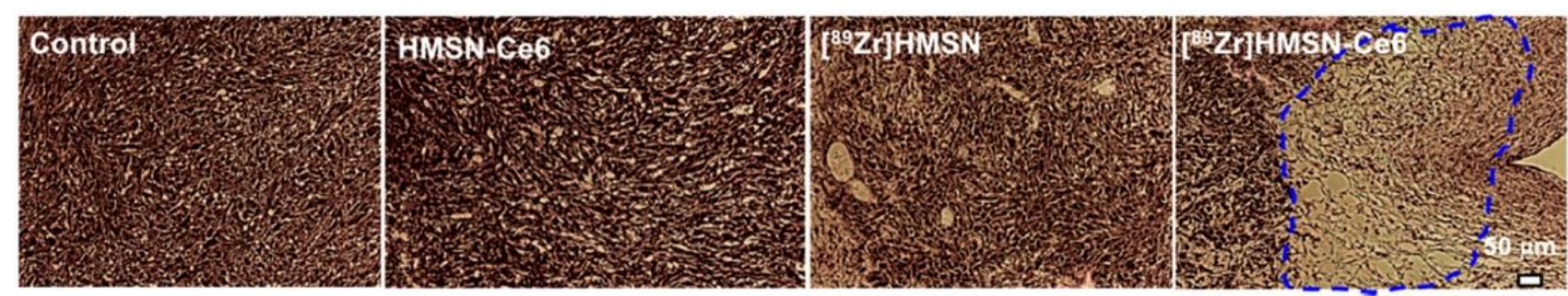
Figure 6. Results of Kamkaew et al., 2016, showing an improved therapeutic effect in 4T1 mammary adenocarcinoma xenografts with Cherenkov radiation activation of the photosensitizer Chlorin e6 loaded in hollow mesoporous silica nanoparticles ([ $\left.\left.{ }^{89} \mathrm{Zr}\right] \mathrm{HMSN}-\mathrm{Ce} 6\right)$ with the incorporation of the positron-emitting radionuclide ${ }^{89} \mathrm{Zr}$. Reprinted with permission from ref. 137. Copyrights 2016 American Chemical Society.

\subsection{Silica nanoparticles in magnetic resonance imaging}

Similarly as in optical imaging, silica can as a construct in MRI contrast agents (CA) be utilized either as a carrier for molecular paramagnetic (Gd) chelates or as a coating material for magnetic nanoparticle cores. As a third and less explored design strategy, the inorganic silica matrix can also be doped with $\mathrm{Gd}^{3+}$ or $\mathrm{Mn}^{2+}$ ions to render the material MRI active. In certain cases, this approach can be preferred over the incorporation of Gd-chelates, as these are reasonably large molecules and hence require large-pore silica materials to be properly accommodated within the porous matrix parallel to allowing proper water access. Doping of metal ions, on the other hand, occurs within the pore walls and thus, does not acquire any space in the pores that can, consequently, be further exploited for loading of drugs or other active molecular agents. ${ }^{[139]}$

The interest in coating of nanoparticles of other materials with mesoporous silica probably originates from 2006, when Hyeon and co-workers coated hydrophobic iron oxide cores with mesoporous silica shells, ${ }^{[77]}$ thus resulting in a MRI-detectable drug carrier. Since then, this approach has been applied to successfully coat inherently hydrophobic nanocrystals (iron oxides and QDs) with the aim of creating multifunctional (theranostic) or multimodal nanoscopic agents ${ }^{[140]}$ using a similar approach, which employs the structuredirecting agent $C_{n} T A B$ both as pore template as well as phase-transfer agent. This requires the core material being inherently hydrophobic; otherwise the surfactant molecules would orient the wrong way around on the core material, leading to phase separation in aqueous solvent needed to synthesize the silica shell. Later, the mesoporous coating approach was modified to be applicable hydrophilic core materials in a direct synthesis approach, i.e. the porous shells are coated in the same aqueous solvent the core materials are dispersed in in the first place; ${ }^{[81]}$ and even one-pot syntheses where the core is synthesized simultaneously with the porous coating has been demonstrated for Au@MSNs. ${ }^{[141]}$ By creating double-layered mesoporous 
shells, yolk/shell structures can be formed after etching of the middle layer. ${ }^{[142]}$ Before the direct coating of mesoporous shells onto nanoparticulate core materials were reported, it was customary to first coat the nano/microparticle with a thin layer of non-porous silica, where after the coating with a porous shell was more straightforward.

Given that the magnetic parameters are highly dependent on the properties of the magnetic nanoparticles, ${ }^{[143]}$ coating of a porous shell also has some implications on the magnetic properties of the core material. Having radially aligned mesopores, water molecules have access to the core, which is a prerequisite for MRI activity. Nevertheless, due to confinement effects in the nanosized pores, the MRI signal can be significantly increased by addition of a porous layer onto magnetic core materials, the signal enhancement of which is dependent on the pore size. ${ }^{[144]}$ There seems to exist an optimal pore size, after which the pores become large enough for the water molecules to diffuse freely again, with a consequential decrease in magnetization and $T_{2}$ relaxivity. The simultaneous increase in water dispersability and colloidal stability in aqueous suspension further contributes towards the enhanced CA properties. Mesoporous silica-coated hollow manganese oxide nanoparticles have also been developed for $T_{1} M R$ imaging, with the same observations that the presence of a porous shell lead to effective longitudinal $\left(R_{1}\right)$ relaxation enhancement of water protons. ${ }^{[145]}$ The main aim with this approach, however, is further accommodating active molecules such as fluorescent dyes and/or drug molecules in the porous shell; the implications of which also has to be investigated with regard to pore size. ${ }^{[146]}$ Smaller pore sizes lead to fluorescence quenching due to spatial proximity of fluorescent dyes, whereas larger pore sizes accelerates drug release unless the pore openings have been capped via a suitable gating mechanism. Porous layers can also be utilized to maximize the particle surface area for efficient further surface functionalization; either to be able to boost the amount of active molecules that can be coupled to the particle surface ${ }^{[147]}$ or to enhance the colloidal stability of the particle suspension by surface functionalization methods. ${ }^{[76]}$

Given their success in drug delivery, MSNs would be an ideal platform for carrying any small-molecular agents and, in the case of paramagnetic Gd-complexes, still keeping them accessible for the surrounding water via the mesoporous channels. ${ }^{[148-150]}$ With regard to the molecular size of the complex as well as considering the 
water molecule interaction is of paramount importance for achieving MRI activity, their performance as CAs are very sensitive to changes in the pore network architecture, which may alter the water access and diffusion. The influence of the localization of the $\mathrm{Gd}$ chelating agent on the resulting relaxometric properties have been emphasized by localizing the chelating agent either on the external surfaces of MSNs or inside the pores. Indeed, it was found as the Gd (III) complexes actually are silent from a relaxometric point of view when they are localized inside the pore channels, where they are less accessible to water molecules than on the external surface of particles. ${ }^{[151]}$ Carniato et al. studied three Gd (III) complexes based on the 1,4,7,10tetraazacyclododecane chelate (DOTA; monoamide-, DOTA- and DO3A-like complexes) which were anchored onto MSNs functionalized with $\mathrm{NH}_{2}$ groups. ${ }^{[152]}$ Here, the interaction between the chelates and surface functional groups markedly influenced the relaxometric properties, and were drastically influenced when going from ionic $-\mathrm{NH}^{3+}$ to neutral amides. The charged surface species seemed interact strongly with the surface silanols, which resulted in the displacement of a coordinated water molecule and consequently in a decrease of the accessibility of the water to the Gd (III) center. Further, the order of preparation may have marked influence on the relaxometric properties. The chelating agent may be introduced to the material in a co-condensation approach, or via post-grafting. Davis et al. studied time delayed co-condensation versus post-grafting as means of Gd-DOTA incorporation (Figure 7) and found significant differences in relaxometric properties, originating from the location of the Gd-DOTA complex. ${ }^{[153]}$ The authors pointed out the importance of understanding and optimizing the Gd internalization and its influence on image contrast whilst maximizing water exchange. 


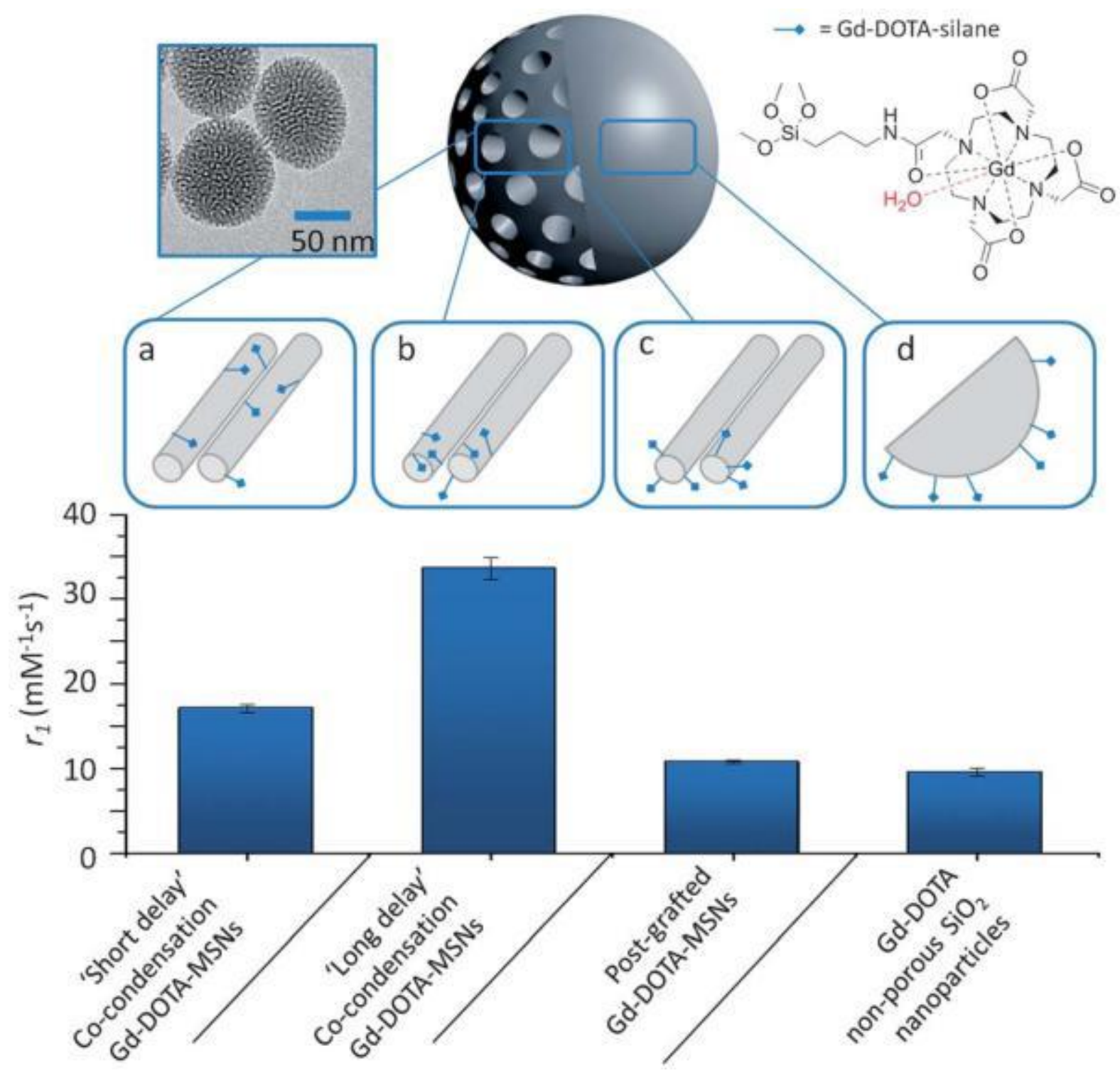

Figure 7. The MSN preparation route influence on the relaxometric properties. Transmission electron microscope image and schematic representation of Gd-DOTA-MSNs (66.3 $\pm 6.6 \mathrm{~nm}$ ) prepared using (a) 'Short delay' co-condensation, where functionalities are internalized deeply in the structure $\left(r_{1}=17.14 \pm 0.49 \mathrm{mM}^{-1} \mathrm{~s}^{-}\right.$ $\left.{ }^{1}\right)$, (b) 'Long delay' co-condensation, where functionalities are internalized nearer to the porous openings $\left(r_{1}=33.57 \pm 1.29 \mathrm{mM}^{-1} \mathrm{~s}^{-1}\right)$ and (c) post-grafting, where functionalities are loaded on external surfaces $\left(r_{1}=10.77 \pm 0.22 \mathrm{mM}^{-1} \mathrm{~s}^{-1}\right)$. (d) Post-grafted Gd-DOTA-non-porous silica nanoparticles $\left(r_{1}=9.56 \pm 0.47 \mathrm{mM}^{-1} \mathrm{~s}^{-1}\right)$. The chart displays $r_{1}$ relaxivities of corresponding nanoparticle samples, measured at 7 T. Reprinted with permission from ref. 153. Copyrights 2012 The Royal Society of Chemistry. 
While covalent conjugation of Gd-chelates to the MSN matrix is probably the most common approach for constructing MSN-based CAs for MRI, this approach is also associated with certain drawbacks. First, the complexation of $\mathrm{Gd}$ to the chelates is a slow process for achieving the stable form of the complex, ${ }^{[154-158]}$ whereby the complexation can take place either before incorporation of the chelate to the MSNs, or the chelate can be incorporated to the MSNs by co-condensation or post-grafting before Gd complexation. ${ }^{[159-}$ ${ }^{161]}$ Further, while complexation of the metal center into a solid support-anchored chelate becomes more cumbersome as compared to the complexation of freely floating molecular agents, ${ }^{[162]}$ the complexation for chelate-grafted solid silica nanoparticles has been reported to being conducted for up to 5 days in the literature for achieving stable complexes. ${ }^{[163]}$ Similar time-frames are generally not applicable for MSNs, since long exposure times to aqueous media can lead to considerable structural deformation in MSN matrices. ${ }^{[100]}$ In parallel, detachment of the chelates upon dissolution of the MSN matrix is also likely during the Gd complexation process, since this takes place under aqueous conditions. ${ }^{[164]}$ Pre-complexing the Gd may, then again, induce hydrolysis of functional groups intended for further conjugation to MSNs if carried out in aqueous solvent. Further, covalent conjugation of molecular agents is generally associated with preferential accumulation on the particle surface, which can lead to blocking the pore openings (post-grafting methods) or being embedded in the MSN matrix in an inaccessible manner (co-condensation). From a CA point of view, such scenarios may lead to the chelates in the pores not being active from a relaxometric point of view due to restricted diffusion of water molecules. Further use of the pores for drug loading, whereby considerably larger (such as drug) molecules than water would need to be accommodated in the pores also becomes a challenge. Consequently, alternative approaches i.e. direct doping of the silica matrix with paramagnetic Gd centers have been attempted. Incorporating $\mathrm{Gd}^{3+}$ ions into the silica matrix results in CAs with a high relaxivity because of the high payload of $\mathrm{Gd}^{3+}$ attainable and the slow tumbling motion of the resulting particles. ${ }^{[165]}$ Guillet-Nicolas et al. ${ }^{[166]}$ prepared MSN based CAs with 3D and 2D pore network connectivity by introducing the $\mathrm{Gd}$ (III) ions into MSNs by the incipient wetness technique, thus creating $\mathrm{GdSi}_{x} \mathrm{O}_{y} \mathrm{MSN}$ hybrid systems. Especially the 3D GdSixOy-MSNs provided a significant increase in ${ }^{1} \mathrm{H}$ longitudinal relaxivity $\left(18.5 \mathrm{~s}^{-1} \mathrm{mM}^{-1}\right.$, i.e. 4.6 times higher than Gd-DTPA) and low $r_{2} / r_{1}$ ratios (1.56) indicative of proper positive MRI CAs. Şen 
Karaman et al. ${ }^{[139]}$ investigated both the influence of preparation parameters and structural properties on the relaxometry of Gd-incorporated MSNs, and also here a pore-expanded MSN structure with a hollow interior (corresponding to better 3D connectivity) showed the most prominent T1 relaxivity (13.1 $\mathrm{mM}^{-1} \mathrm{~s}^{-1}$ and a $r_{2} / r_{1}$ ratio of 1.6$)$. When the Gd was incorporated already in the synthesis step, the hollow poreexpanded MSNs resulted in a roughly 20-fold higher value compared to the corresponding regular pore-sized MSN. However, when the Gd was incorporated after the MSN synthesis, the most effective $r_{1}$ value enhancement was obtained depending on SDA removal method, whereby calcination resulted in almost 6fold higher $r_{1}$ values as compared to solvent-extraction of the corresponding MSN matrix. Notably, the Gd ${ }^{3+}$ amount in all cases was below $1 \mu \mathrm{mol} \mathrm{mg}{ }^{-1}$ whereby the Gd (III) concentration exposed to cells labeled with the CA was only in the range of $30 \mu \mathrm{M}$ (c.f. Magnevist ${ }^{\circledR}$ typically used as $500 \mathrm{mM}$ ). Hollow structure has also shown to influence the MR imaging capability of manganese oxide particles as compared to solid manganese oxide nanoparticles, ${ }^{[167]}$ and manganese-impregnated MSNs have also been prepared as MRI CAs. ${ }^{[168]}$

\subsection{In vivo MR imaging of silica nanomaterials}

After MRI cell tracking was introduced in 1993 to follow the cell survival and migration ${ }^{[169]}$, the first time in vivo time course of cell migration was reported in $2001 .{ }^{[170]}$ In the literature, particulate CAs, mainly iron oxide nanoparticles, are employed frequently for this purpose. SPIONs have become the preferred CAs for

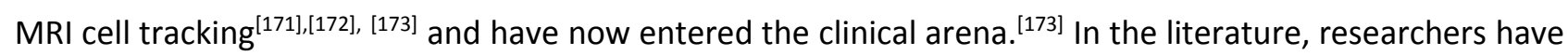
been investing time for novel design of such particulate CAs instead of using them as native constituents, since the native SPIONs appear to suffer from low intracellular labelling efficiency once it is aimed to be used for tracking of cell fate. ${ }^{[174]}$ Hence, several modifications have been investigated to improve the cellular internalization of these materials. Among them, mesoporous silica take part in the core@shell design of SPIONS in which a silica framework has been coated around the SPION core. As already discussed above, this particular design allows incorporation of other imaging probes into the contrast agent for dual imaging modalities, and also eases the surface functionalization of nanoparticles for bioconjugation and targeting. The pointed out strategy has been reported for the first time by Lu et al. ${ }^{[175]}$ In their study, they have 
designed fluorescein isothiocyanate (FITC)-incorporated silica-coated SPION, core@shell, SPION@SiO 2 (FITC), as a bifunctional magnetic vector that has been used to label human mesenchymal stem cells (hMSCs). The labelled hMSCs were efficiently labelled with these nanoparticles and monitored in vitro and in vivo with a clinical 1.5 T MRI. ${ }^{[176]}$ However, the produced hypointensities (dark regions) on T2/T2*-weighted MR images which presumably indicate the location of the transplanted cells with the SPIONS, cannot be distinguished from other hypointense regions, such as hemorrhage and blood clots. Therefore, researchers have started to employ the refered design of core@shell with also T1 relaxation agents. In a recent study of Kim et al similar core@shell design strategy was employed for the enhancement of $\mathrm{T} 1$ contrast of native $\mathrm{MnO}$ cores. ${ }^{[79]}$ In

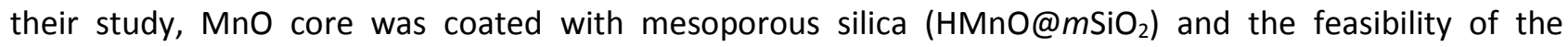

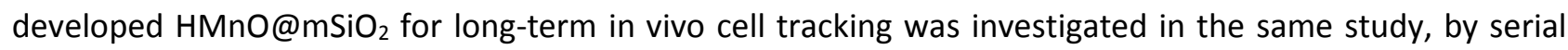
monitoring the fate of transplanted and labelled multipotent mesenchymal stem cells (MSCs) with MRI.

Silica materials have also been employed as constitute of contrast agents for blood-pool imaging (also known as intravascular imaging). This ultra-high spatial resolution MR imaging requires in vasculature remained contrast agents that leads to prolonged reduction $\mathrm{T} 1$ of blood for sensitive MR detection, and define the diagnostic data of cancer and diseases of the cardiovascular system. Blood-pool agents are designed to prolong the imaging window and possess a much higher relaxivity compared to extracellular agents in order to improve vessel-to-background signal. In this context, mesoporous silica material is considered to provide an ideal platform for the development of MR-enhancing hybrid materials due to their high surface areas and tunable pores. The accessibility of the magnetic center to water molecules is considered as the key for developing efficient nanoparticulate MR contrast agents which can be readily maintained with the mentioned features of mesoporous silica, especially with the tunable pore size opportunities in their designs. In the literature, starting with the grafting of Gd chelates onto mesoporous silica, many attempts were carried out for designing highly efficient blood-pool contrast agents. ${ }^{[177], ~[161], ~[178]}$ In the study of Lin and coworkers the effectiveness of MSN grafted Gd-chelates as in vivo MR contrast agents was evaluated by the administration of $2.1 \mu \mathrm{mol} \mathrm{kg}{ }^{-1}$ body weight in mice upon tail vein injection, and T1 weighted enhancement 
was clearly observed in aorta of the mouse after 15 minutes from the injection. Similar design of Gd chelating agent DTPA coupled silica was employed for the tracking of implanted hMSCs with nanoparticles into the midline high brain stem of a nude mouse after labelling of cells with the nanoparticles. The efficiency of designed CA for the tracking of stem cells was stated based on the observed bright dots for 14 days in the MR images of mouse with the absence of hMSCs migration. ${ }^{[159]}$

Although the abovementioned design of Gd (III) chelators coupled to the silica nanoparticles have been used frequently as $\mathrm{CAs}$ in in vivo $\mathrm{MRI}$, self-assembled $\mathrm{Gd}_{2} \mathrm{O}_{3}$ molecules inside the mesopores of silica has also been used in the literature for similar applications. In the study of Shao et al, $\mathrm{Gd}_{2} \mathrm{O}_{3}$ incorporated MSNs, $\mathrm{Gd}_{2} \mathrm{O}_{3} @ \mathrm{SiO}_{2}$ was prepared. ${ }^{[179]}$ In vivo MR imaging of these particles demonstrated strong MRI contrast enhancement in nasopharyngeal carcinoma (NPC) xenografted tumors. In their study, they have also suggested that the prepared $\mathrm{Gd}_{2} \mathrm{O}_{3} @ \mathrm{SiO}_{2}$ nanoparticles may be adopted as a blood pool agent for studying variations in organ blood volume and capillary permeability, since they have demonstrated prolonged blood circulation time compared to DTPA chelates of Gd (III). Choa and co-workers have presented the contrast ability of their designed $\mathrm{Gd}_{2} \mathrm{O}_{3} @ \mathrm{SiO}_{2}$, core@shell structured nanocomposite with in vitro and in vivo MRI analysis. ${ }^{[180]}$ Furthermore, they have also aimed for neutron capture therapy with their designed nanocomposite upon its poly(2-methacryloyloxyethyl phosphorylcholine) (PMPC) corona coating as outer layer of composites. Their work revealed that $\mathrm{Gd}_{2} \mathrm{O}_{3} @ \mathrm{SiO}_{2} @ P M P C$ nanocomposite has excellent accumulation and sustentation in tumor cells. Consequently, the obtained results in the literature reveal that MSN based contrast agents are not only highly efficient CAs for MR imaging, but can also be developed as probes for unique applications such as potential targeted probes for in vivo molecular imaging of cancer.

\subsection{PET and SPECT imaging of silica nanomaterials}

One of the great advantages of PET and SPECT imaging is their quantitativeness and sensitivity, allowing the dynamic observation and kinetic analysis of the accumulation of the DDSs in the target region. However, the short half-lives of many of the PET and SPECT radionuclides, such as ${ }^{11} \mathrm{C}\left(\mathrm{t}_{1 / 2}=20.3 \mathrm{~min}\right),{ }^{18} \mathrm{~F}\left(\mathrm{t}_{1 / 2}=109.7 \mathrm{~min}\right)$ 
and ${ }^{99 \mathrm{~m}} \mathrm{Tc}\left(\mathrm{t}_{1 / 2}=6.0 \mathrm{~h}\right)$ have limited their use for the investigation of only the early accumulation of the nanotheranostic agents (Table 1). However, homing of the nanotherapeutics into a tumor may take hours or even several days, depending on the targeting approach and circulation time of the system. Longer living positron and gamma emitting isotopes, such as ${ }^{64} \mathrm{Cu}\left(\mathrm{t}_{1 / 2}=12.7 \mathrm{~h}\right),{ }^{89} \mathrm{Zr}\left(\mathrm{t}_{1 / 2}=78.4 \mathrm{~h}\right)$ and ${ }^{124} \mathrm{I}\left(\mathrm{t}_{1 / 2}=4.18 \mathrm{~d}\right)$ have been used for DDSs with slower pharmacokinetics and for the investigation of their biodistribution and tumor accumulation over several days.

The group of Prof. Weibo Cai is one of the pioneering groups in the utilization of PET methods in evaluation of silica based DDSs. In one of their first studies they reported the evaluation of doxorubicin loaded mesoporous silica nanoconjugates, ${ }^{64} \mathrm{Cu}-\mathrm{NOTA}-\mathrm{mSiO2}-\mathrm{PEG}-\mathrm{TRC} 105$, in $4 \mathrm{~T} 1$ tumor bearing mice. ${ }^{[181]}$ TRC105 is an antibody binding to the endoglin receptor which is highly overexpressed in angiogenic endothelial cells and upregulated under hypoxic conditions. Functionalization of the nanoconjugates with the TRC105 antibody yielded in successful targeted accumulation in the tumor peaking at $5 \mathrm{~h}$ post-injection (p.i.) (5.9 \pm $0.4 \% \mathrm{ID} / \mathrm{g})$. The same model and targeting strategy was utilized for quantitative evaluation of distribution and tumor accumulation of doxorubicin loaded hollow mesoporous silicon nanoparticles (HMSN) by using a multimodality approach. ${ }^{[182]}$ HMSNs are promising new DDSs with large cavity for hosting the drug inside a silica shell. The investigated HMSN system exhibited 3-15 times higher doxorubicin loading capacity when compared to the conventional MSN systems. The tumor accumulation of the evaluated ${ }^{64} \mathrm{Cu}-\mathrm{HMSN}-\mathrm{ZW} 800-$ TRC105 nanosystems peaked at $9.9 \pm 0.9 \%$ ID/g at $4 \mathrm{~h}$ p.i. and NIRF imaging (Ex $\vee 465 \mathrm{~nm}, \mathrm{Em} v 580 \mathrm{~nm}$ ) was successfully used for confirming in vivo enhanced doxorubicin delivery into tumor. Even higher tumor uptakes were reported recently for biodegradable [ ${ }^{89} \mathrm{Zr}$ ]bMSN-PEG5k-TRC105 particles in the same model. ${ }^{[183]}$ The bMSN particles were radiolabeled by using intrinsic radiolabeling via coordination of ${ }^{89} \mathrm{Zr}^{4+}$ to the silanol groups of the porous silica framework, after which the particles were functionalized with PEG (5kD) and the TRC105 antibody. $11.4 \pm 2.1 \% \mathrm{ID} / \mathrm{g}$ of $\left.{ }^{89} \mathrm{Zr}\right] \mathrm{bMSN}-\mathrm{PEG} 5 \mathrm{k}-\mathrm{TRC} 105$ was observed in tumor at $6 \mathrm{~h}$ (p.i.), which is one of the highest uptake values reported for the silica based DDSs so far. The highest tumor-tomuscle ratio was obtained at $24 \mathrm{~h}$ p.i. $(47.18 \pm 7.19)$. Modelling of the tumor accumulation by using a twocompartment model gave an elimination half-life $\left(t_{1 / 2 \beta}\right)$ of $4.6 \mathrm{~h}$. Despite of the excellent targeting to the 
tumor, still most of the $\left[{ }^{89} \mathrm{Zr}\right] \mathrm{bMSN}-\mathrm{PEG} 5 \mathrm{k}-\mathrm{TRC} 105$ were sequestered by the macrophages and phagocytes in liver and spleen (40-45 \%ID/g at $48 \mathrm{~h}$ p.i.) (Figure 8). Slightly lower tumor uptakes were reported for vascular endothelial growth factor (VEGF) receptor targeted ${ }^{64}$ Cu-NOTA-MSN-PEG-VEGF121 (7.8 $\pm 0.2 \%$ ID/g at $3 \mathrm{~h}$ p.i.) in an U87MG glioma model. ${ }^{[184]}$

A first-in-human trial was reported for the ultra-small 6-7 nm hybrid core@-shell silica nanoparticles, called Cornell dots (C-dots), after labeling them with ${ }^{124}{ }^{\left[{ }^{[20]}\right.}$ The hybrid particles were inherently fluorescent, containing the dye Cy5. The C-dots were functionalized with integrin targeted peptide cyclo-(Arg-Gly-AspTyr) (cRGDY) to detect integrin-expressing lesions in pre- and intra-operative conditions. The ${ }^{124}$ I-cRGDYPEG-C dot nanoconstructs were investigated in a microdosing study as a hybrid PET-optical imaging agent for lesion detection, cancer staging, and treatment management in humans. PET evaluation allowed accurate measurement of the fraction of the injected particles at tumor sites and monitoring of the time-dependent particle uptake in the tumor. ${ }^{124}$ I-CRGDY-PEG-C dot whole-body clearance half-time values varied between 13 to 21 hours, resulting to effective dose between $0.115-0.228 \mathrm{mSv} / \mathrm{MBq}$. When using DDSs with larger diameter, the whole-body clearance half-life may increase significantly due to the more effective sequestration of the DDSs from the circulation by liver and spleen. When combined with the long half-lives of radioisotopes such as ${ }^{89} \mathrm{Zr}$ or ${ }^{124} \mathrm{l}$, this increases the unnecessary radiation exposure to the patient and may hamper the clinical translation of the nanotheranostic DDS. Pretargeted PET and SPECT imaging based on bioorthogonal chemical reactions has been investigated for solving this downside. In the pretargeted approach the DDS is traced by a small molecular radiotracer in vivo based on specific chemical reaction between the DDS and the tracer (see also 3.4 and Figure 14). This makes it possible to trace DDSs with long distribution half-life with a tracer with fast pharmacokinetics and allows the use of short living radionuclides for radiolabeling of the tracer. Altogether it minimizes the duration of the radiation exposure to the patient, decreasing significantly the effective dose gained during the imaging study. One of the early studies reporting use of bioorthogonal reactions for tracing MSNs was reported in 2013 by Kim et al. ${ }^{[185]}$ They used the strainpromoted alkyne azide cycloaddition (SPAAC) for tracing aza-dibenzocyclooctyne (DBCO) functionalized MSNs in U87MG tumor-bearing mice with $\left[{ }^{18} \mathrm{~F}\right]$ fluoropentaethylene glycolic azide. In a more recent study, 
Mikula et al. reported pretargeted PET imaging of trans-cyclooctene modified MSN particles by using a ${ }^{11} \mathrm{C}$ labeled tetrazine. ${ }^{[186]}$ The TCO-modified MSN particles were successfully traced in lungs of the mice pretreated with the particles 5 minutes prior to the tracer injection.

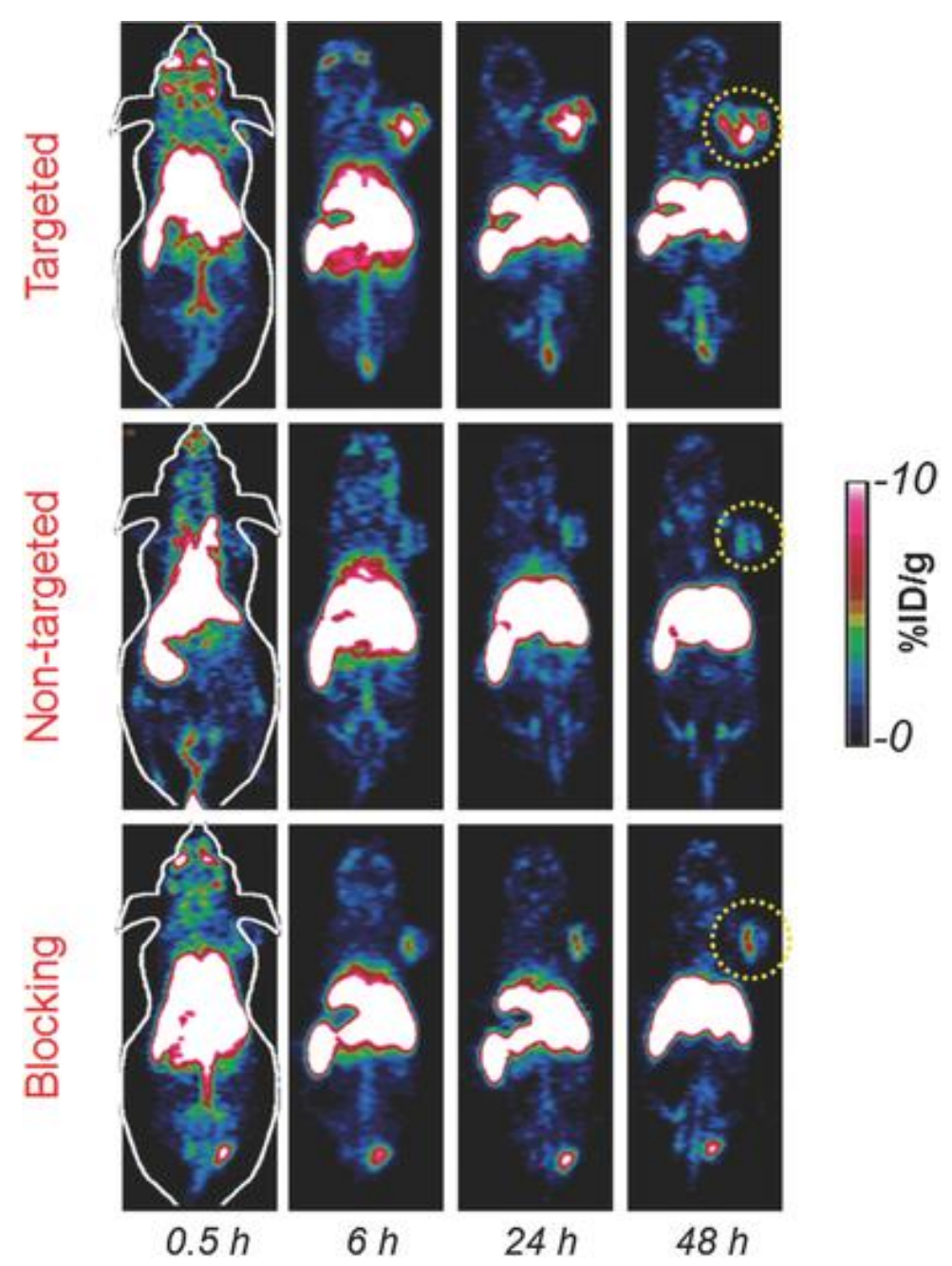

Figure 8. In vivo tumor vasculature targeting. Representative tumor-bearing coronal slices from serial PET scans. Targeted group: $\left.{ }^{89} \mathrm{Zr}\right] \mathrm{bMSN}-\mathrm{PEG5k}$-TRC105; non-targeted group: $\left[{ }^{89} \mathrm{Zr}\right.$ ]bMSN-PEG5k and blocking group: ${ }^{89} \mathrm{Zr}$ ]bMSN-PEG5k-TRC105 (with a pre-injected blocking dose of TRC105). Yellow circles indicate the location of 4T1 breast tumor. Reprinted with permission from ref. 183. Copyrights 2016 John Wiley and Sons. [183] 
A multitude of different imaging modality combined probes has been constructed where silica has played a role, due to its flexibility in design allowing the combination of all strategies described above. For example, Kumar et al. ${ }^{[29]}$ reported the usage of $20-25 \mathrm{~nm}$, near-infrared (NIR) fluorophore- and radiolabeled ORMOSIL nanoparticles for in vivo optical and PET imaging, and also investigated their biodistribution, clearance, and toxicity. There are a multitude of examples of nanoprobes that combine MRI with OI, which has been suggested as one of the appealing combinations. ${ }^{[187],[188],[189]}$ Similarly as in the other multimodal nanoprobe studies researchers have also investigated the effects of dual MRI + OI nanoprobes in in vivo tests by considering the design parameters of the nanoprobes. Jang et al have prepared differently sized core@shell designed nanoprobes with varying shell thicknesses. ${ }^{[190]}$ The multimodal nanoprobes encompass an iron oxide core coated with a silica shell onto which fluorescent Cy dyes were conjugated to produce multimodal nanoparticular imaging probes. According to their findings, the weakest fluorescence signal was obtained in the subcutaneously injected of SCC7 xenografted mice imaging by the performed NIRF tomographic imaging for the fluorescent Cy coupled core@shell nanoparticles when the silica shell was the thinnest, due to distance-dependent quenching/dequenching behavior of fluorescent $\mathrm{Cy}$ dye coupled on the core@shell nanoparticles. Thus, the relative signal enhancement was $40 \%$ for the case of the nanoprobes with the thinnest silica shell and 30\% for nanoprobes with the thickest silica shell, respectively. Researchers have also successfully demonstrated the feasibility of real-time diagnostic ultrasound (US) and MRI modalities with silica-based multimodal nanoprobes to be used in high-intensity focused ultrasound (HIFU) surgeries. In the study reported by Chen et al, ${ }^{[191]}$ they have doped a hollow mesoporous silica structure (H-MSN) with highly dispersed manganese oxide species to make them suitable as CAs for efficient T1-weighted MR imaging for accurate HIFU guidance. With the assistance of the prepared nanoprobe, they could precisely locate the targeted tumour tissue in the liver of rabbits, and also obtained an enhanced synergistic therapeutic. In the study of Lee et al., ${ }^{[140]}$ multiple magnetite nanocrystals conjugated dye-doped MSNs were fabricated for their use as a vehicle capable of simultaneous magnetic resonance and fluorescence imaging as well as drug delivery. In vivo investigations revealed that these multifunctional nanocomposite nanoparticles were delivered to the tumor sites via passive targeting. The nanoparticles served as successful multimodal 
imaging probes and simultaneously delivered anticancer drugs to the tumor site. Chen and co-workers have also demonstrated the success of simultaneous non-invasive cancer diagnosis and efficient chemotherapy by their design based on $\mathrm{H}-\mathrm{MSN}$ incorporated with $\mathrm{Mn}^{2+}$ that they called hybrid mesoporous composite nanocapsules (HMCNs), capable of dual US and pH-responsive MR imaging (Figure 9). ${ }^{[192]}$ In conclusion, while the furthermost advantage with multimodal probes is providing complementary information in one shot, e.g. high resolution and high sensitivity; nanoscopic imaging agents are foreseen to be invaluable constructs in theranostic nanomedicines for personalized treatment in the future. ${ }^{[193]}$
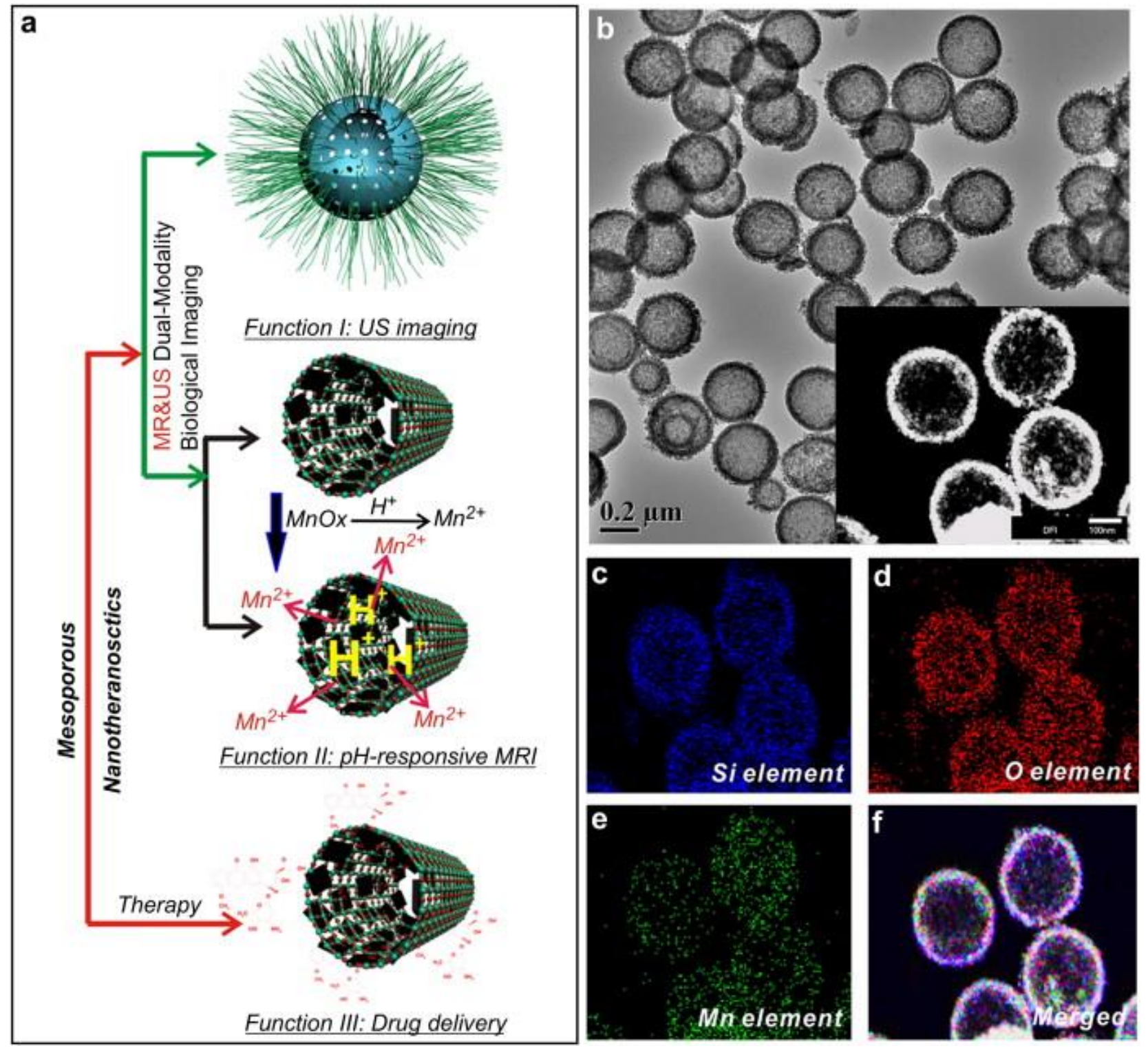

Figure 9. Overview of the versatility and characteristics of a multimodal nanoprobe with theranostic potential. (a) Schematic illustration of the microstructure and structure-related theranostic functions of the 
HMCNs designed by Chen et al. (b) TEM image of HMCNs (inset: STEM image with scale bar $=100 \mathrm{~nm}$ ); $(\mathrm{c}-\mathrm{f}$ ) Elemental mapping of $\mathrm{Si}(\mathrm{c}), \mathrm{O}(\mathrm{d})$ and $\mathrm{Mn}(\mathrm{e})$ in $\mathrm{HMCNs}$ (f: color-merged image of $\mathrm{c}$, d and e). Reprinted with permission from ref. 192. Copyrights 2012 Elsevier.

\section{In vivo imaging with silicon based materials}

\subsection{Fabrication and properties of porous silicon (PSi)}

Porous silicon (PSi), the porous form of the semiconductor element silicon, was discovered somewhat serendipitously during electrolytic shaping of silicon disks in the 1950s. ${ }^{[194,195]}$ PSi was largely dismissed as a curiosity at the time, but interest towards the material quickly spiked in the 1990s with the reports on the mechanism of the porous layer formation in the electrochemical etching process and subsequent luminescence of PSi arising from a combination of a quantum confinement effect and surface defects ${ }^{[196-198]}$, and the biocompatibility of the material. ${ }^{[199]}$ Reports on the ability of PSi to improve the dissolution of poorly soluble drugs by their retention in an amorphous state when confined to the narrow channel network, to protect sensitive payloads, and to support the growth of cells quickly ensued, directing the use of PSi towards biomedical and drug delivery applications, reviewed in depth by Anglin et al. ${ }^{[200]}$, and Santos et al. ${ }^{[201]}$ Today, PSi is still predominantly prepared by the original electrochemical etching method with hydrofluoric acid in ethanol, rendering a porous layer on the surface of the silicon wafer that can be lift-off by abruptly increasing the etching current, and subsequently processed to nanoparticles by ultrasonic fracture and ball-milling with size fractionation by sieving and ultracentrifugation (Figure 10). ${ }^{[202]}$ One of the hallmarks of PSi is the tunability of the material properties, i.e. porous network density and pore dimensions, by the conditions used in the electrochemical etching process. However, as a top-down nanomaterial, PSi nanoparticles are often irregular in shape and initially polydisperse, and consequently, alternative methods, such as photolithography can be employed to generate PSi nanoparticles of uniform size and shape. ${ }^{[203-205]}$ Morphology of PSi based drug delivery systems and their surface chemistry influences biological fate of the material in the body. The freshly prepared, "as-anodized" surface of PSi is terminated with silyl hydrogens 
$\left(\mathrm{Si}-\mathrm{H}_{\mathrm{x}}\right.$; where $\left.\mathrm{x}=1-3\right)$, necessitating chemical surface modification for the facile conjugation of optical and radioactive labels and targeting biomolecules. The principal surface-modification methods for PSi include thermal oxidation ${ }^{[206]}$, and thermal carbonization ${ }^{[207]}$ and hydrocarbonization ${ }^{[208]}$, the latter two of which render the material extremely resistant to a variety of harsh chemical conditions and "aging" by atmospheric oxygen. ${ }^{[200]}$ Lately, amine-modification with 3-aminopropyltriethoxysilane $(\mathrm{APTES})^{[209]}$ and undecylenic acid $^{[210]}$ for EDC/NHS coupling chemistry and alkyne-modification for Cu-catalyzed click chemistry ${ }^{[211]}$ have gained popularity for the conjugation of biomolecules, labels, and polymers to PSi. In pH above 7 PSi degrades to non-toxic orthosilicic acid, which is excreted to urine. The degradation rate of PSi particles depends on their surface chemistry and porosity. PEGylation and silylation by aminopropyl-triethoxysilane (APTES) has been shown to decrease the degradation rate. ${ }^{[212]}$ Several methods have been used for assessing biological fate of PSi. However, clinical applications of PSi as nanotheranostic systems are still to come. Quantification of tissue silicon content from ex vivo harvested organs by using ICP-OES or ICP-MS techniques has provided valuable information about blood circulation time and biodistribution of PSi nano- and microparticles with different surface modifications. ${ }^{[213-216]}$ 


\section{Crystalline Si}

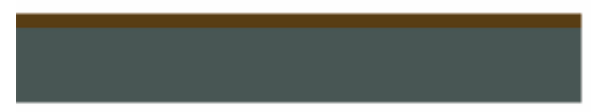

$$
\text { He+ }
$$

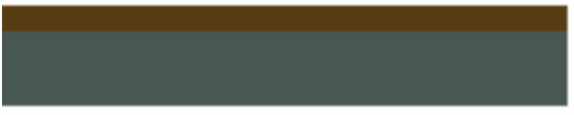

\section{Growth of PSi layer}
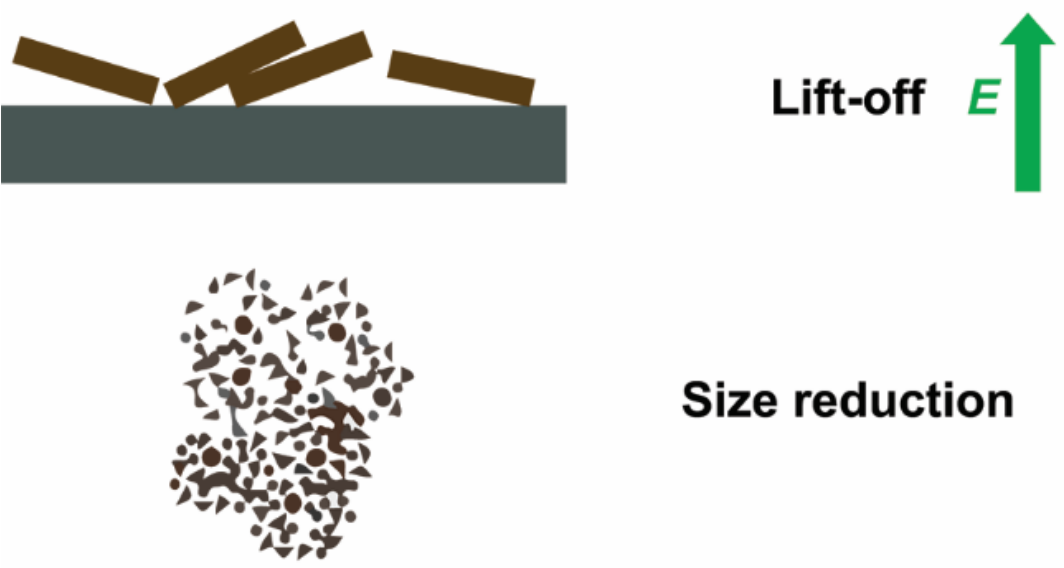

\section{Size reduction}

\section{Sieving or ultracentrifugation}

Figure 10. Preparation of PSi particles starts with the electrochemical etching of crystalline silicon wafers in an ethanolic solution of hydrofluoric acid, whereby the porous layer grows in the direction of the etching current $(E)$. The degree of porosity and the thickness of the porous layer can be finely controlled by the conditions of the electrochemical etch. The porous layer is lift off the crystalline Si substrate by an abrupt increase in the etching current, and the resulting PSi reduced to nano- and microparticles typically by ball milling and ultrasonic fracture followed by sieving and ultracentrifugation to isolate the desired size fraction.

\subsection{Optical imaging probes based on PSi}

PSi exhibits luminescence in the wavelength region of visible light at room temperature, and in the nearinfrared (NIR) region when excited. ${ }^{[217]}$ While both modes of luminescence are suitable for preclinical optical 
imaging, NIR luminescence is superior in its depth of penetration in tissues and is, consequently, more amenable to clinical translation. The intrinsic luminescence of PSi, however, has found use in many biosensor applications, including in the monitoring of drug release in vitro, and upon PSi ophthalmic implant dissolution in vivo in the vitreous of the eye. ${ }^{[52,218]}$ Most biosensing applications of PSi are based on an 'on/off'-type shift in the reflectance spectrum of the material as a result of the absorption of the desired biomolecule or change in material surface chemistry, for example upon dissolution. The use of luminescent PSi in biosensing applications has been recently reviewed extensively by Jenie and co-workers, to which the reader is referred ${ }^{[219]}$. "True" in vivo luminescent imaging applications of PSi (meaning without the use of additional fluorescent labels, these approaches are discussed below) have been limited to date, although as Park et al. have showed the luminescence of PSi to permit longitudinal tracking of PSi with a widely available preclinical optical imaging system. ${ }^{[53]}$ Figure 11 shows the excitation-based fluorescence imaging of the PSi nanoparticles in the study by Park et al. in cells and mice. Recent advancements in optical imaging techniques have led to significant improvements in the sensitivity of the technique by enabling the differentiation of the tissue autofluorescence from the photoluminescent emissions of the PSi. ${ }^{[220]}$ This has been made possible by the procedure of time-gating (TG), in which the long emission half-life of photoluminescent PSi nanoparticles is utilized for identifying and discarding the shorter-lived autofluorescent emissions originating from the surrounding tissue. With TG contrast improvements to $>100$-fold has been reported improving significantly translational potential of the photoluminescent PSis. ${ }^{[221]}$ Recently, Osminkina and co-workers reported the preparation of highly luminescent PSi nanowires from heavily boron-doped substrates ${ }^{[222]}$, advancing the possibility to use these for efficient in vivo imaging in the future. However, despite these inherent luminescent properties of PSi, covalent surface modification with fluorescent labels is predominantly used for optical imaging of PSi in cell and animal models, likely due to the limitations to available surface chemistries in luminescent PSi. Additionally, luminescence alone might not permit the imaging of PSi nanoparticles at very low concentrations in tissues, and fluorochromes are often used instead to generate PSi imaging probes for both microscopy and preclinical imaging. Labels incorporated covalently to PSi to date include FITC, Alexa Fluor ${ }^{\circledR} 488^{[223]}$, and the NIR dye DyLight $750 .{ }^{[213]}$ 

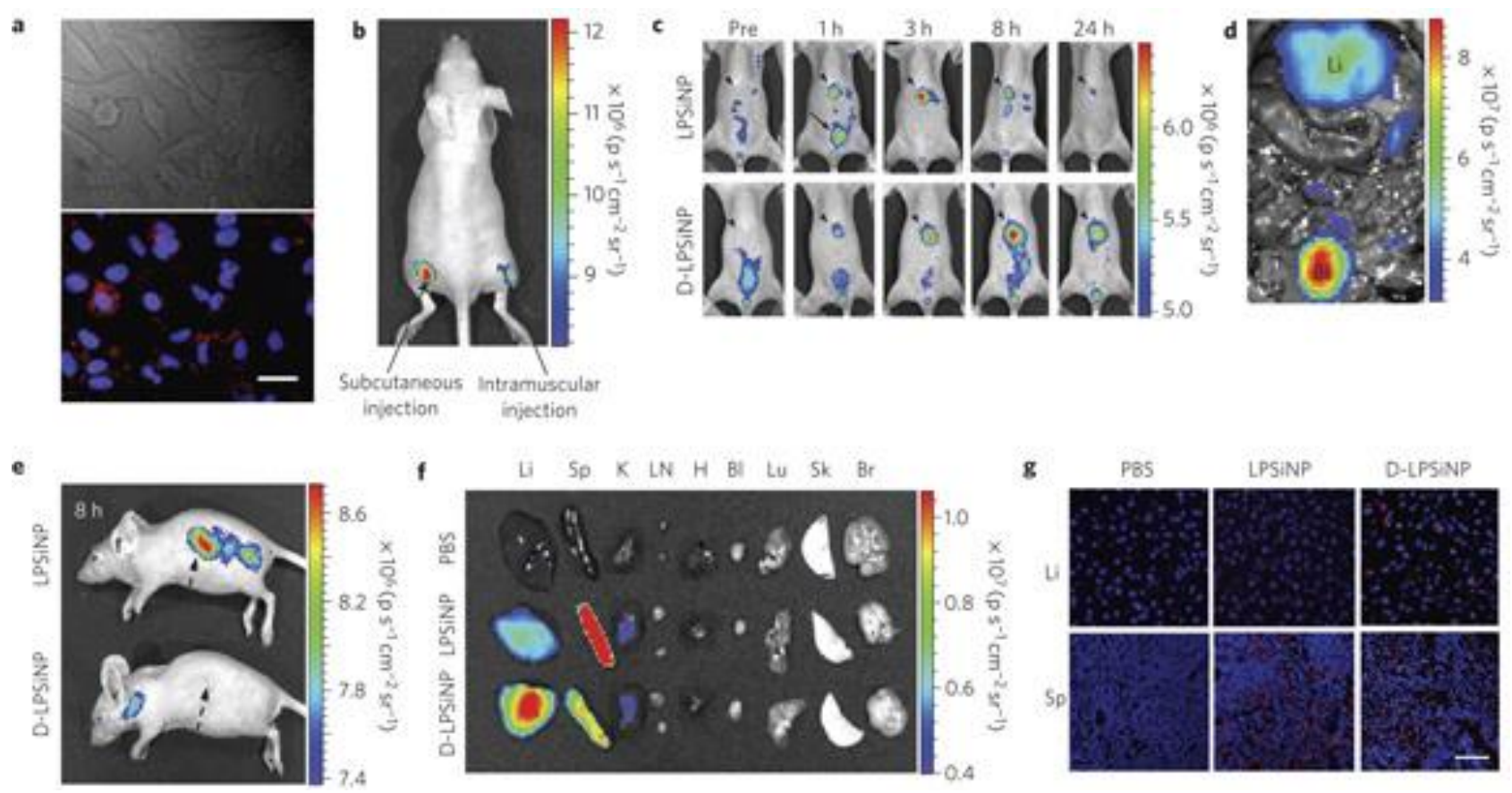

Figure 11. Excitation-based fluorescence imaging of luminescent porous silicon nanoparticles (LPSiNP) in cells and both in vivo and ex vivo in MDA-MB-231 tumor-bearing mice in a pioneering study by Park et al. illustrates the potential of the material for the generation of optical imaging probes with simultaneous drug delivery capability. Reprinted with permission from ref. 53. Copyrights 2009 Nature Publishing Group.

\subsection{MRI imaging of porous silicon}

Porosity of the PSi allows construction of multifunctional vectors for imaging by entrapping smaller contrast agents such as super-paramagnetic SPIONs inside the porous structure. The approach has allowed observation of PSi accumulation into major organs and cancerous tissue by using MRI, but the in vivo MRI applications of PSi have remained more scarce than reported for silica based materials. ${ }^{[224,225]}$ Serda et al. investigated SPION loaded PSi as a platform for MRI traceable vaccines. The intravenously administered hybrid nanoparticles accumulated in spleen as could be observed as increased negative contrast in the T2 weighted images. Paramagnetic elements are amenable also for guiding drug delivery systems into tumor by using remote magnetic guidance. Gizzatov et al. successfully used the remote magnetic guidance for enhancing accumulation of super-paramagnetic discoidal mesoporous silicon particles (SiMPs) into melanoma tumors as observed as an increased contrast in a T2-weighted MRI. ${ }^{[225]}$ Intravenously injected Gd- 
DOTA conjugated SiMP nanoconstructs accumulated in ovarian tumors up to $2 \% \mathrm{ID} / \mathrm{g}$. The uptake was quantified from the harvested tissue by using ICP-MS. ${ }^{[216]}$ In a recent study, influence of dual PEGylation to blood circulation half-life and spleen accumulation of iron oxide nanocrystal loaded PSi NPs (DPEG-MaPSi) was successfully investigated by using dynamic MRI. ${ }^{[226]}$ Particle accumulation to liver was monitored by following decrease of $\mathrm{T}_{2} *$ after intravenous injection of the particle suspension to rat $(1 \mathrm{mg} /$ animal) (Figure 12). Native silicon contains $4.6 \%$ of ${ }^{29} \mathrm{Si}$, with a spin $1 / 2$-nucleus and amenable for hyperpolarization in a magnetic field. This allows label-free imaging of silicon particles with improved sensitivity by using MRI. Depolarization of ${ }^{29} \mathrm{Si}$ in the silicon lattice is not affected by surface functionalization of the particles, which allows use of different targeting strategies without affecting sensitivity of the detection. Recently, as low as $4.2 \times 10^{-13} \mathrm{~mol} \mathrm{I}^{-1}$ particles molar sensitivity was reported by using hyperpolarized ${ }^{29} \mathrm{Si}-\mathrm{MRI}$ and a cylindrical phantom containing a suspension of high-purity silicon particles. ${ }^{[227]}$ The hyperpolarized ${ }^{29} \mathrm{Si}-\mathrm{MRI}$ technique was successfully applied for detection of APTES functionalized and PEGylated silicon particles in vivo in mice after different administration routes (oral, intraperitoneal, and intravenous).
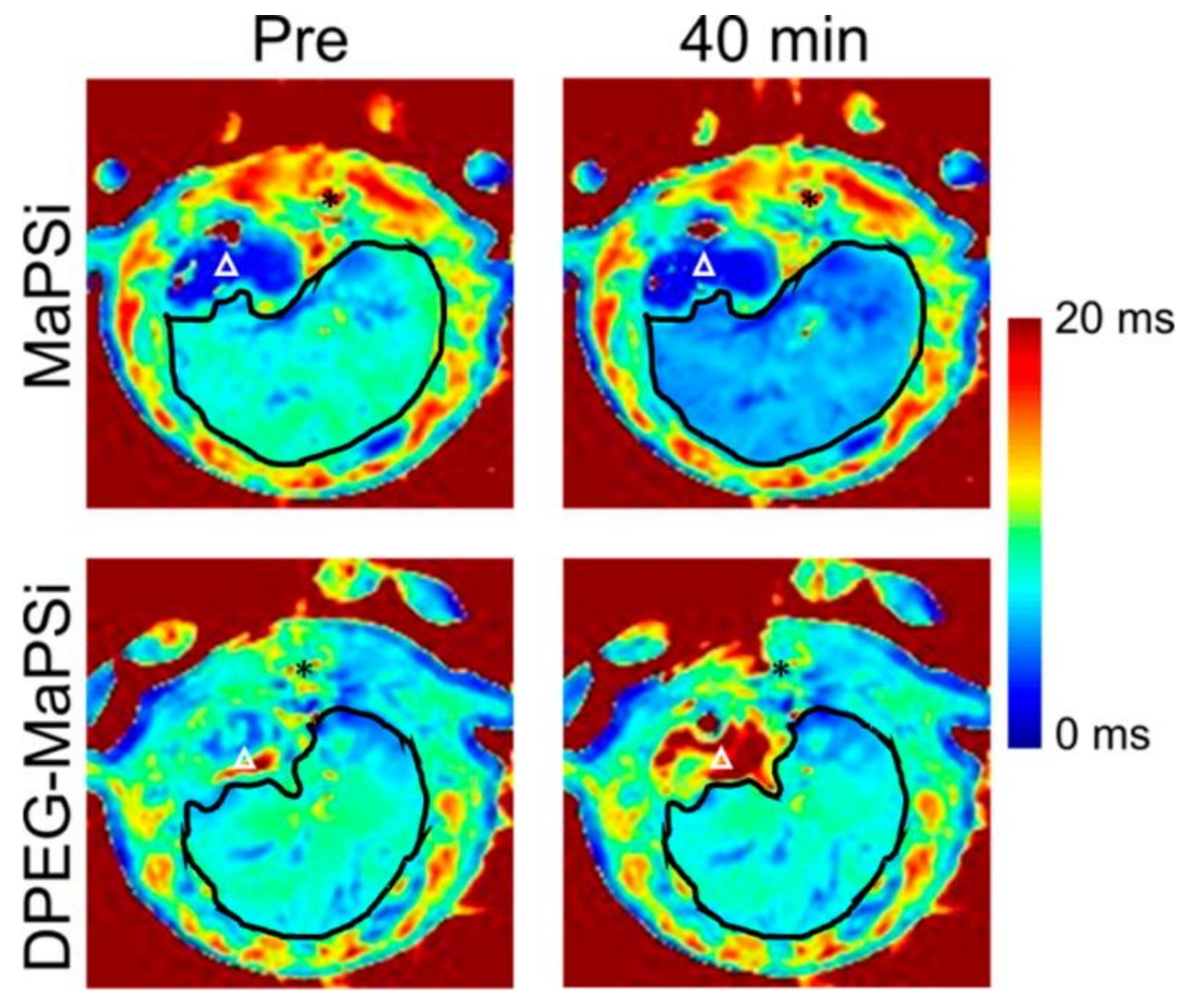
Figure 12. MRI T2* map of a rat liver before and $40 \mathrm{~min}$ after the $2 \mathrm{~mL}, 0.5 \mathrm{mg} / \mathrm{mL}$ injections of MaPSi or DPEG-MaPSi nanoparticles. The liver is circled with a black line, the white triangle designates the cardiac part of the stomach, and the black asterisk shows the spinal cord. Subcutaneous fat is seen as longer relaxation times. The four spots above the body of the rat are from the water-heated pad. Reprinted with permission from ref. 226. Copyrights 2016 American Chemical Society.

\subsection{PET and SPECT imaging porous silicon}

The direct method for radiolabeling of porous silicon by ${ }^{18} \mathrm{~F}$ has proved to be a valuable tool in studying the in vivo properties of PSi based drug delivery systems. ${ }^{[42]}$ Because the labeling reaction is based on a substitution reaction between silicon and a non-carrier-added $\left[{ }^{18} \mathrm{~F}\right]$ fluoride, there is no need for any functionalization of the surface for the labeling. This ensures minimal effect due to the labeling to biological properties of the material. The only limitation is that the material needs to be passivated against a hydrolytic attack. In one of the pioneering studies, the biodistribution of thermally hydrocarbonized PSi nanoparticles (THCPSi) was determined in rats after administration of $\left[{ }^{18} \mathrm{~F}\right]$ THCPSi nanoparticle suspension either intravenously, subcutaneously or via oral gavage. The study provided quantitative results on PSi nanoparticle distribution up to six hours after their administration. ${ }^{[228]}$ After intravenous administration, the $\left[{ }^{18} \mathrm{~F}\right] \mathrm{THCPSi}$ particles accumulated in the liver and spleen, indicating fast elimination from the circulation typical for nanomaterials without any stealth effect-promoting surface functionalization. The orally given $\left[{ }^{18} \mathrm{~F}\right] \mathrm{THCPSi}$ nanoparticles did not cross the intestinal wall and reached the distal parts of the colon within $6 \mathrm{~h}$. After subcutaneous injection $\left[{ }^{18} \mathrm{~F}\right]$ THCPSi NP resided in the subcutaneous space for at least up to $4 \mathrm{~h}$ post-injection without any significant diffusion from the administration site. The same methodology was utilized for investigating the influence of different surface modifications to the biodistribution of thermally hydrocarbonized PSi. Coating of ${ }^{18}$ F-labeled THCPSi with an amphiphilic surface-active fungal hydrophobin (HFBII) protein was found to exhibit only a minor influence on the blood circulation time of the system, although it greatly stabilized the nanoparticle suspension in physiological media. ${ }^{[229]}$ However, after oral administration the HFBII coating significantly increased the transit time of the particles from the stomach to 
the small intestine. ${ }^{[230]}$ The protein coating was found to be responsive to the surface-active compounds in the duodenum and quickly dissolved when exposed to intestinal fluid. Therefore, HFBII exhibited great potential as a mucoadhesive protein for the construction of gastroretentive drug delivery systems. Coating of THCPSi nanoparticles with glyceryl monostearate, L- $\alpha$-phospatidyl choline, polyvinyl alcohol (PVA) and PEG 6000, by a solid-in-oil-in-water (S/O/W) method provided THCPSi-SLNC particles, which had improved stability against aggregation in aqueous solutions, and increased smoothness of the surface. ${ }^{[231]}$ This was reported to improve the cytocompatibility, and to prolong drug dissolution as compared to non-coated THCPSi particles. When evaluated in breast cancer xenografts, the solid lipid coated $\left[{ }^{18} \mathrm{~F}\right] \mathrm{THCPSi}$ nanoparticles exhibited improved accumulation into the tumor when compared to the non-coated NPs (tumour-to-liver ratio $0.10 \pm 0.08$ and $0.24 \pm 0.09$ for ${ }^{18} \mathrm{~F}$-THCPSis and ${ }^{18} \mathrm{~F}$-THCPSi-SLNCs, respectively). ${ }^{[232]}$ The observed accumulation of the ${ }^{18} \mathrm{~F}$-THCPSi-SLNCS to the tumor was mediated by the EPR effect and no tumor-targeting moieties were utilized. For that reason, the total amount of the ${ }^{18} \mathrm{~F}$-THCPSi-SLNC remained modest $(0.081 \pm$ $0.036 \%$ of the injected dose per gram of tissue, $\% \mathrm{ID} / \mathrm{g}$ ). Influence of dextran coating on the biodistribution of ultra-small silicon quantum dots, $\left({ }^{64} \mathrm{Cu}-\mathrm{QDs}, \mathrm{D}_{h}=15.1 \pm 7.6 \mathrm{~nm}\right)$ was investigated after radiolabeling them with ${ }^{64} \mathrm{Cu} .{ }^{[233]}$ The PET image analysis in mice revealed rapid blood clearance (only $4.3 \%$ ID/g in blood after 2 $\mathrm{min})$. As expected by the small size of the particles, the main accumulation was observed in urinary bladder, but also significant amount of the ${ }^{64} \mathrm{Cu}-\mathrm{QD}$ s were sequestered by the liver.

As for many other nanomaterials, PEGylation of the PSi surface has been found to improve their stealth properties and to lengthen their circulation residence time. However, not as drastic effects have been observed as for other materials, and the potential of PEGylation to improve the circulation half-life has remained modest. In the first reported study with undecylenic acid modified THCPSi nanoparticles (UnTHCPSi), the surface undecylenic acids were conjugated with tyrosine residues allowing radiolabelling of the surface with a SPECT-compliant radioisotope ${ }^{125}$ I by electrophilic radioiodination. ${ }^{[234]}$ The resulting ${ }^{125}$ I-PSi nanoparticles were then O-PEGylated (10 kDa) and biodistribution of the O-PEGylated PSi nanoparticles was determined in healthy mice after intravenous injection. The $10 \mathrm{kDa}$ PEG was clearly not able to mask the particles from the RES recognition and the particles were quickly sequestered by the liver and spleen, 
resulting in only $2 \% \mathrm{ID} / \mathrm{g}$ in the blood after the first ten minutes. Histological evaluation of the liver sections revealed that the nanoparticles were located mainly in the lumen of the liver blood vessels and were able to emanate also into the sinusoids. A more successful example on improving PSi blood-circulation time by PEGylation can presented by comparing two separate studies in which a heart-targeting atrial natriuretic peptide (ANP) functionalized UnTHCPSi NPs were investigated by using ${ }^{111}$ In-labeled NPs and SPECT/CT imaging. ${ }^{[23][236]}$ The biodistribution of the ANP modified PEGylated (Un-P-D-ANP) and non-PEGylated [111 In]UnTHCPSi NPs was monitored in a rat model of heart ischemia, which had been induced by an isoprenaline injection $24 \mathrm{~h}$ prior to the experiment. At $10 \mathrm{~min} 0.9 \%$ of the injected non-PEGylated particles were observed in the heart as quantified based on SPECT image analysis. PEGylation was able to increase blood circulation half-life and at the same time point $4.5 \%$ of the injected PEGylated ANP PSi (Un-P-D-ANP) particles were found in the heart. The longer circulation half-life improved targeting efficiency resulting to significantly improved heart accumulation of the ANP functionalized PSi particles $(p<0.01)$ when compared to the control particles bearing no targeting peptide (Un-P-D) (Figure 13). The endocardium specific accumulation of the $\left[{ }^{111} \mathrm{In}\right]$ Un-P-D-ANP was confirmed by autoradiography analysis of the cardiac cryosections.
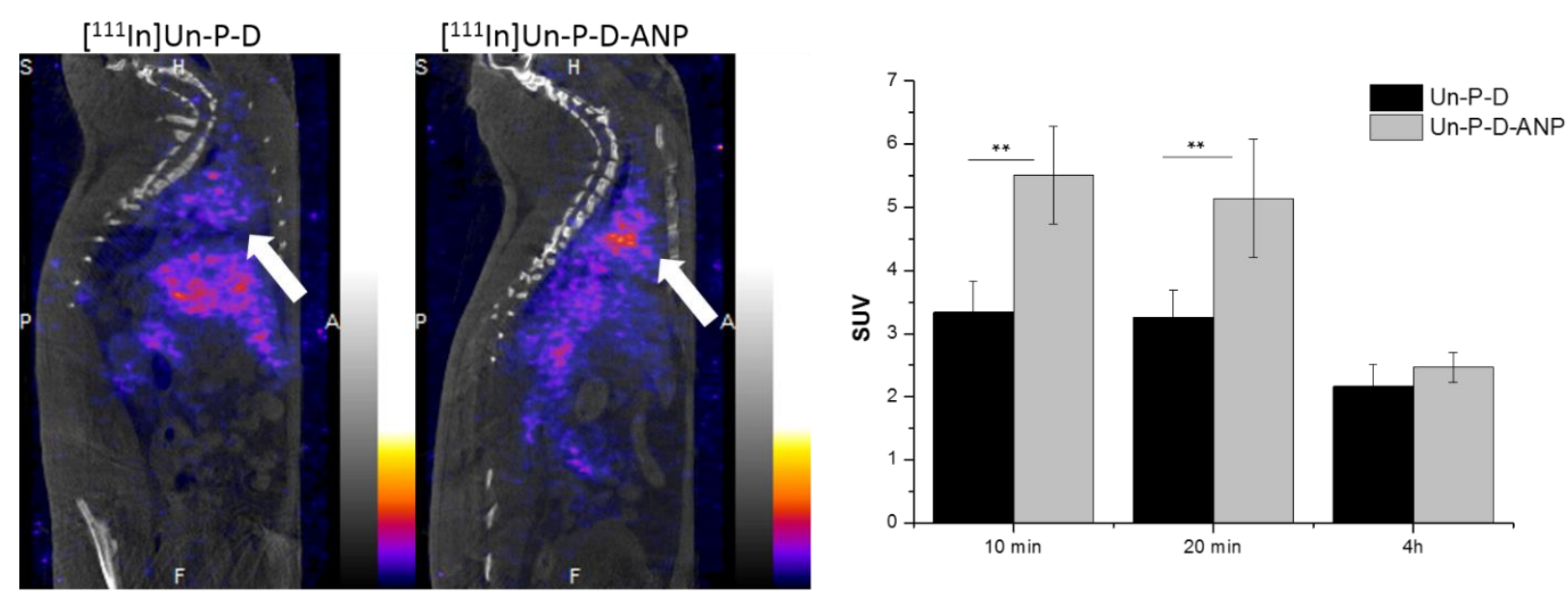

Figure 13. Representative sagittal SPECT/CT images showing biodistribution of intravenously administered PEGylated [111 In]UnTHCPSi nanoparticles at 10 min time point in rat with induced myocardial infraction. 
$\left[{ }^{111}\right.$ In] Un-P-D-ANP NPs were targeted to the infracted myocardium by using an ANP peptide. SUV in heart the control NPs and [111 In]Un-P-D-ANP NPs at $10 \mathrm{~min}, 20 \mathrm{~min}$ and $4 \mathrm{~h}$ time points. Values are represented as mean \pm s.d. $(n=4)$. Adapted with permission from ref. 236. Copyrights 2017 John Wiley and Sons.

Influence of iRGD-functionalization to PSi biodistribution and therapeutic efficiency was investigated by using

${ }^{111}$ In-labeled UnTHCPSi nanoparticles, loaded with an anti-angiogenic drug sorafenib. The sorafenib loaded nanoconstructs were evaluated in a survival study after their iv. and intratumoral administration in an ectopic PC3-MM2 mouse xenograft model and by using SPECT/CT imaging. ${ }^{[237]}$ iRGD is a cyclic tumor targeting and tissue penetrating peptide, which has been identified by phage display technology. iRGD peptide functionalization enhanced the tumor uptake of the NPs when administered intravenously. However, the tumor growth suppression effect was similar to the free sorafenib through intravenous administration. SPECT/CT imaging and ex vivo biodistribution analysis revealed that despite of the improved tumor targeting efficiency $(0.19 \pm 0.06 \% \mathrm{ID} / \mathrm{g})$, the total amount of the nanoconstructs was still too modest for efficient targeted therapy. Instead, after intratumoral delivery the NPs were retained in the tumor, resulting efficient inhibition on tumor growth with particle-loaded sorafenib compared to the free drug.

In a recent study, PEGylated PSi NPs were traced in vivo by using pretargeted PET imaging based on biorthogonal chemical reaction between a ${ }^{18}$ F-labeled tracer and the NPs (Figure 14). ${ }^{[238]}$ In the study, the recognition between the tracer $\left(\left[{ }^{18} \mathrm{~F}\right] \mathrm{TAF}\right){ }^{[239]}$ and the PSi NPs was based on inverse electron-demand DielsAlder cycloaddition (IEDDA) between the ${ }^{18} \mathrm{~F}$-labeled tetrazine derivative and trans-cyclooctene (TCO) modified PSi-NPs. PET evaluation revealed that the IEDDA reaction was fast, and high radioactivity accumulation in spleen was observed already during the first 10 minutes after administration of the tracer. The formed cycloaddition product was stable and no significant decrease in radioactivity levels in spleen was observed during the 120 minutes observation time. Highest target-to-background-ratio was achieved at 120 min after the tracer injection. Kinetic analysis of the spleen accumulation confirmed irreversible binding of the tracer at the PSi accumulation site in spleen. However, binding in liver was found reversible, indicating 
incomplete reaction either due to fast racemization of the TCO groups at PSi in liver or internalization of the PSi NPs converting them inaccessible to the hydrophilic non-internalizing $\left[{ }^{18} \mathrm{~F}\right] \mathrm{TAF}$ tracer.
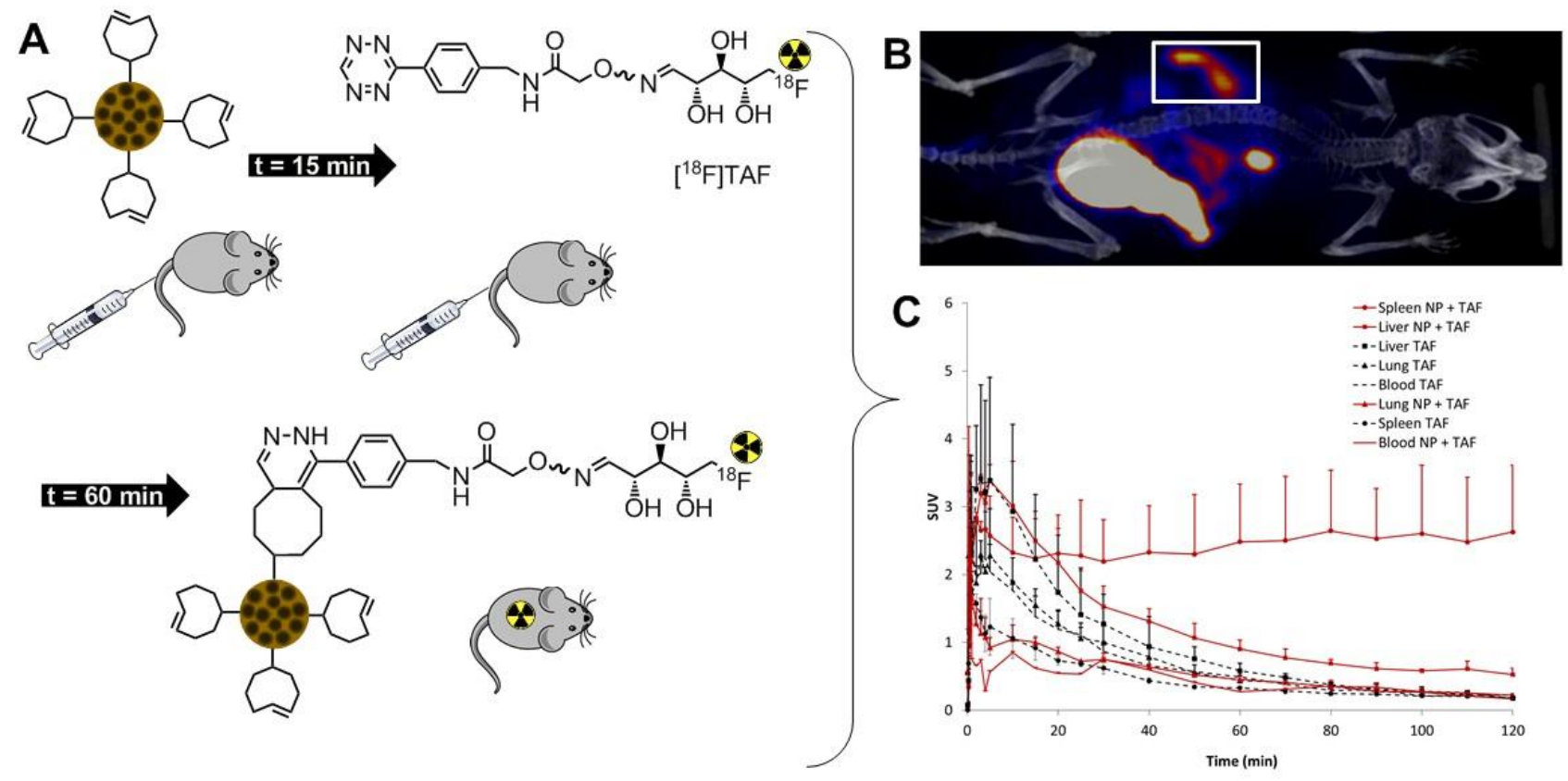

Figure 14. Pretargeted PET imaging of TCO-PSi NPs in mice. A. The TCO-PSi NPs were injected 15 minutes before injection of the tracer $\left[{ }^{18} \mathrm{~F}\right]$ TAF. PET imaging was performed 60 minutes after injection of the tracer. B. The TCO-PSi NPs were traced in spleen (delineated by a box). Some accumulation also in liver was observed. The observed high levels of radioactivity in gall bladder, intestines and urinary bladder are caused by elimination of the $\left[{ }^{18} \mathrm{~F}\right] \mathrm{TAF}$ and its radioactive metabolites. $\mathrm{C}$. The time-activity-curves (TAC) revealed that the IEDDA reaction was fast, with high radioactivity accumulation in spleen already during the first 10 minutes p.i. The cycloaddition product was stable and no significant decrease in radioactivity levels in spleen was observed during the 120 minutes observation time. Adapted with permission from ref. 238. Copyrights 2017 American Chemical Society.

\section{Conclusions}

Significant progress has been made in the development of drug delivery systems based on silica and silicon nanostructures over the last decades. Methods have been established for constructing non-porous and porous silica and silicon nanoparticles with well-defined and tunable surface chemistry allowing flexible 
functionalization of their surfaces with different organic functional groups and conjugation of targeting moieties. Despite the great progress, site-specific delivery of therapeutics by targeted nanosystems remains a challenge, and even for the best of DDSs most of the intravenously injected nanovectors end up in the liver and spleen. In vivo imaging has demonstrated its value in the evaluation of methods for improving sitespecific nanotherapeutic delivery. Methods have been developed for tagging silica and silicon nanoconstructs with various labels, such as superparamagnetic and paramagnetic elements, radionuclides or fluorochromes, allowing for their tracking with MRI, PET, SPECT or optical imaging methods. Fluorescent silica nanoparticles have already found an established place in especially biomedical research, being avidly exploited not only in in vitro bioimaging, but also biosensing and biological labeling. For this reason, such non-porous nanoparticles are also commercially available with a range of different detection wavelengths. The intrinsic luminescence of PSi and the ability of MSN to coordinate metals inside the matrix have been utilized for the construction of nanoprobes with minimal disturbance on the surface chemistry and, consequently, the in vivo behavior of the labeled constructs over non-labeled ones. Core@shell design have been especially advantageous in the design of two-in-one fluorescent-magnetic nanocomposites for multimodal applications. In the core@shell design, a silica coating can form an effective barrier between the magnetic core and the fluorophore preventing quenching of the fluorescence. The same strategy can be applied to protect core materials from the surroundings, which could otherwise lead to inactivation of the detectable signal, e.g. quenching of UNCP luminescence by water. The recent development of pretargeted approaches for PET and SPECT imaging enables the use of radioisotopes with a short physical half-life for tracing the DDSs with slow pharmacokinetics, thereby reducing the effective radiation dose imparted to the subject by their administration. Additionally, pretargeted imaging obviates the radiolabeling of the NPs before their administration, greatly simplifying the nanoparticle preparation and handling processes.

In multimodality imaging, the advantages of different imaging modalities are combined in order to gain optimal imaging tracer performance in terms of sensitivity, resolution and quantitation. Multimodality approaches have been essential in understanding the interactions between nanotherapeutics and biological systems, and have increased our knowledge on the mechanisms behind successful targeting, transport and 
extravasation of the nanoconstructs to the target site. Integration of the diagnostic function into a DDS enables the development of nanotherapeutics for clinical applications to monitor the drug delivery process during the treatment, as well as the individual's response to therapy. Multimodal imaging is most widely applied for achieving precise localization of DDS accumulation by combining the signal originating from the imaging probe (OI, SPECT or PET) and the anatomical information gained from the CT or MRI image. Applications in which two or more modalities are combined into the same material are still less frequent but emerging. The great advantage in the integration of the different modalities into the same material is the possibility to combine the strengths of different imaging techniques. PET is a sensitive and fully quantitative imaging modality limited by its relatively poor spatial resolution. MRI and OI methods allow high spatial resolution but, despite the recent advances in these imaging techniques, MRI still lacks in the sensitivity and Ol in the quantitativity. Multimodality nanoprobes are needed for the improved visualization of tissue function and morphology in pathological conditions and upon therapeutic interventions. For example, in oncological applications a tumor-targeting PET/OI multimodality probe would allow the sensitive detection of the tumor and its metastases by using PET/CT imaging, while the high sensitivity and spatial resolution of the optical methods enable the confirmation of the complete removal of the tumor tissue during the surgical operation. In intraoperative optical imaging, the attenuation of the optical signal in the tissue can be overcome by bringing the detector closer to the region of interest even in the deeper tissue allowing for the detection of the cancer cells stained by the fluorescent multimodality probe. The multimodality nanoprobes described herein have their most significant benefit in research, development and clinical evaluation of new investigational DDS nanoconstructs. However, their utility in clinical diagnostics and intraoperative cancer care still needs to be demonstrated. The often complex and expensive production of many multimodality nanoconstructs is a challenge for clinical translation, as for clinical diagnostic agents a balance between the costs and the benefit to the patient should be maintained.

Silica and silicon are biocompatible, biodegradable and versatile platforms for the development of nanotheranostic probes and many of the methods developed during the discovery of silica and silicon based DDSs warrant clinical translation. With the rapid advancement of these materials into biomedical research, 
the selection of designs that can be created have become multifold. In parallel, the characterization methods and tests needed for the investigation of the material behavior biological systems have multiplied, and as with any nanoscopic drug delivery system, the biggest challenge lies therein. Standardized procedures for the biological evaluation for bringing nanoformulations closer to the clinic are still lacking, posing a serious impediment to their development. Still, the entry of the silica-based C-dots in clinical trials suggests that there is considerable future promise for Si-based materials in clinical practice. This applies especially to multimodal nanosystems utilizing the translational in vivo imaging modalities, such as PET, SPECT and MRI, but also optical imaging probes have high potential for finding clinical utility in intraoperative imaging. As versatile synthetic platforms both porous silica and silicon lend themselves excellently for the development of translatable multimodal nanotheranostic systems.

\section{References}

[1] E. Forssen, Z. Tökés, Proc Natl Acad Sci USA 1981, 78, 1873.

[2] P. Guaglianone, K. Chan, E. DelaFlor-Weiss, R. Hanisch, S. Jeffers, D. Sharma, F. Muggia, Invest. New Drugs 1994, 12, 103.

[3] E. Blanco, H. Shen, M. Ferrari, Nat Biotech 2015, 33, 941.

[4] R. E. Serda, E. Blanco, A. Mack, S. J. Stafford, S. Amra, Q. Li, A. van de Ven, T. Tanaka, V. P. Torchilin, J. E. Wiktorowicz, M. Ferrari, Mol. Imaging 2011, 10, 7290.2011.00008.

[5] V. Lenaerts, J. F. Nagelkerke, T. J. C. Van Berkel, P. Couvreur, L. Grislain, M. Roland, P. Speiser, J. Pharm. Sci. 1984, 73, 980.

[6] J.-C. Leroux, F. De Jaeghere, B. Anner, E. Doelker, R. Gurny, Life Sci. 1995, 57, 695.

[7] S. M. Moghimi, J. Szebeni, Prog. Lipid Res. 2003, 42, 463.

[8] R. A. Petros, J. M. DeSimone, Nat. Rev. Drug Discov. 2010, 9, 615.

[9] F. Alexis, E. Pridgen, L. K. Molnar, O. C. Farokhzad, Mol. Pharm. 2008, 5, 505.

[10] W. Jiang, KimBetty Y. S., J. T. Rutka, ChanWarren C. W., Nat Nano 2008, 3, 145.

[11] H. Zhang, K. E. Burnum, M. L. Luna, B. O. Petritis, J.-S. Kim, W.-J. Qian, R. J. Moore, A. HerediaLangner, B.-J. M. Webb-Robertson, B. D. Thrall, D. G. Camp, R. D. Smith, J. G. Pounds, T. Liu, PROTEOMICS 2011, 11, 4569.

[12] B. Godin, C. Chiappini, S. Srinivasan, J. F. Alexander, K. Yokoi, M. Ferrari, P. Decuzzi, X. Liu, Adv Funct Mater 2012, 22, 4225.

[13] T. Yu, D. Hubbard, A. Ray, H. Ghandehari, J. Controlled Release 2012, 163, 46.

[14] Y. Matsumura, H. Maeda, Cancer Res. 1986, 46, 6387.

[15] E. A. Azzopardi, E. L. Ferguson, D. W. Thomas, J. Antimicrob. Chemother. 2013, 68, 257.

[16] C. N. Marti, M. Gheorghiade, A. P. Kalogeropoulos, V. V. Georgiopoulou, A. A. Quyyumi, J. Butler, J. Am. Coll. Cardiol. 2012, 60, 1455.

[17] H. Cabral, Y. Matsumoto, K. Mizuno, Q. Chen, M. Murakami, M. Kimura, Y. Terada, M. R. Kano, K. Miyazono, M. Uesaka, N. Nishiyama, K. Kataoka, Nat. Nanotechnol. 2011, 6, 815.

[18] B. D. Chithrani, W. C. W. Chan, Nano Lett. 2007, 7, 1542.

[19] F. Lu, S.-H. Wu, Y. Hung, C.-Y. Mou, Small 2009, 5, 1408. 
[20] E. Phillips, O. Penate-Medina, P. B. Zanzonico, R. D. Carvajal, P. Mohan, Y. Ye, J. Humm, M. Gönen, H. Kalaigian, H. Schöder, H. W. Strauss, S. M. Larson, U. Wiesner, M. S. Bradbury, Sci. Transl. Med. 2014, 6, 260 ra149.

[21] M. Benezra, O. Penate-Medina, P. B. Zanzonico, D. Schaer, H. Ow, A. Burns, E. DeStanchina, V. Longo, E. Herz, S. Iyer, J. Wolchok, S. M. Larson, U. Wiesner, M. S. Bradbury, J. Clin. Invest. 2011, 121, 2768.

[22] C.-H. Heldin, K. Rubin, K. Pietras, A. Ostman, Nat Rev Cancer 2004, 4, 806.

[23] C. Le Roy, J. L. Wrana, Nat. Rev. Mol. Cell Biol. 2005, 6, 112.

[24] G. W. Gould, J. Lippincott-Schwartz, Nat. Rev. Mol. Cell Biol. 2009, 10, 287.

[25] L. Y. T. Chou, K. Ming, W. C. W. Chan, Chem. Soc. Rev. 2011, 40, 233.

[26] L. Wasungu, D. Hoekstra, J. Controlled Release 2006, 116, 255.

[27] E. Dauty, J.-S. Remy, G. Zuber, J.-P. Behr, Bioconjug. Chem. 2002, 13, 831.

[28] Z. Wang, C. Tiruppathi, R. D. Minshall, A. B. Malik, ACS Nano 2009, 3, 4110.

[29] R. Kumar, I. Roy, T. Y. Ohulchanskky, L. A. Vathy, E. J. Bergey, M. Sajjad, P. N. Prasad, ACS Nano 2010, 4, 699.

[30] K. Y. Choi, G. Liu, S. Lee, X. Chen, Nanoscale 2012, 4, 330.

[31] P. Das, A. Saha, A. R. Maity, S. C. Ray, N. R. Jana, Nanoscale 2013, 5, 5732.

[32] P. Debbage, W. Jaschke, Histochem. Cell Biol. 2008, 130, 845.

[33] J.-L. Coll, Nanomed. 2011, 6, 7.

[34] T. Desmettre, J. M. Devoisselle, S. Mordon, Surv. Ophthalmol. 2000, 45, 15.

[35] S. T. Proulx, P. Luciani, S. Derzsi, M. Rinderknecht, V. Mumprecht, J.-C. Leroux, M. Detmar, Cancer Res. 2010, 70, 7053.

[36] J. Yu, D. Javier, M. A. Yaseen, N. Nitin, R. Richards-Kortum, B. Anvari, M. S. Wong, J. Am. Chem. Soc. 2010, 132, 1929.

[37] J. Merian, J. Gravier, F. Navarro, I. Texier, Molecules 2012, 17, 5564.

[38] C. Tassa, S. Y. Shaw, R. Weissleder, Acc. Chem. Res. 2011, 44, 842.

[39] F. Chen, S. Goel, H. F. Valdovinos, H. Luo, R. Hernandez, T. E. Barnhart, W. Cai, ACS Nano 2015, 9, 7950.

[40] F. Chen, H. F. Valdovinos, R. Hernandez, S. Goel, T. E. Barnhart, W. Cai, Acta Pharmacol. Sin. 2017, 38, 907.

[41] V. Bernard-Gauthier, Wängler, E. Schirrmacher, A. Kostikov, K. Jurkschat, Wängler, R. Schirrmacher, BioMed Res. Int. 2014, 2014, 454503.

[42] M. Sarparanta, E. Mäkilä, T. Heikkila, J. Salonen, E. Kukk, V. P. Lehto, H. A. Santos, J. Hirvonen, A. J. Airaksinen, Mol Pharm 2011, 8, 1799.

[43] A. Louie, Chem. Rev. 2010, 110, 3146.

[44] K.-S. Park, J. Tae, B. Choi, Y.-S. Kim, C. Moon, S.-H. Kim, H.-S. Lee, J. Kim, J. Kim, J. Park, J.-H. Lee, J. E. Lee, J.-W. Joh, S. Kim, Nanomedicine Nanotechnol. Biol. Med. 2010, 6, 263.

[45] L. M. Jurkić, I. Cepanec, S. K. Pavelić, K. Pavelić, Nutr. Metab. 2013, 10, 2.

[46] R. K. Iler, The Chemistry of Silica: Solubility, Polymerization, Colloid and Surface Properties and Biochemistry of Silica, Wiley, 1979.

[47] A. P. Bowditch, K. Waters, H. Gale, P. Rice, E. a. M. Scott, L. T. Canham, C. L. Reeves, A. Loni, T. I. Cox, MRS Online Proc. Libr. Arch. 1998, 536, DOI 10.1557/PROC-536-149.

[48] A. Rosengren, L. Wallman, M. Bengtsson, T. Laurell, N. Danielsen, L. m. Bjursten, Phys. Status Solidi A 2000, 182, 527.

[49] J. G. Croissant, Y. Fatieiev, N. M. Khashab, Adv. Mater. 2017, 29, 1604634.

[50] H. Jaganathan, B. Godin, Nanotoxicity Bench Bedside 2012, 64, 1800.

[51] T. Asefa, Z. Tao, Chem. Res. Toxicol. 2012, 25, 2265.

[52] L. Cheng, E. Anglin, F. Cunin, D. Kim, M. J. Sailor, I. Falkenstein, A. Tammewar, W. R. Freeman, Br. J. Ophthalmol. 2008, 92, 705.

[53] J. H. Park, L. Gu, G. von Maltzahn, E. Ruoslahti, S. N. Bhatia, M. J. Sailor, Nat Mater $2009,8,331$.

[54] S. P. Low, N. H. Voelcker, L. T. Canham, K. A. Williams, Biomaterials 2009, 30, 2873. 
[55] M. A. Tölli, M. P. A. Ferreira, S. M. Kinnunen, J. Rysä, E. M. Mäkilä, Z. Szabó, R. E. Serpi, P. J. Ohukainen, M. J. Välimäki, A. M. R. Correia, J. J. Salonen, J. T. Hirvonen, H. J. Ruskoaho, H. A. Santos, Biomaterials 2014, 35, 8394.

[56] W. Stöber, A. Fink, E. Bohn, J. Colloid Interface Sci. 1968, 26, 62.

[57] G. Kolbe, Das Komplexchemische Verhalten Der Kieselsäiure., Friedrich-Schiller Universitäit, 1956.

[58] I. A. Rahman, V. Padavettan, J. Nanomater. 2012, 2012, 1.

[59] K. S. Finnie, J. R. Bartlett, C. J. A. Barbé, L. Kong, Langmuir 2007, 23, 3017.

[60] T. Yanagisawa, T. Shimizu, K. Kuroda, C. Kato, Bull. Chem. Soc. Jpn. 1990, 63, 1535.

[61] T. Yanagisawa, T. Shimizu, K. Kuroda, C. Kato, Bull. Chem. Soc. Jpn. 1990, 63, 988.

[62] F. Hoffmann, M. Cornelius, J. Morell, M. Fröba, Angew. Chem. Int. Ed. 2006, 45, 3216.

[63] V. Shirshahi, M. Soltani, Contrast Media Mol. Imaging 2015, 10, 1.

[64] S. Li, M. M. Lin, M. S. Toprak, D. K. Kim, M. Muhammed, Nano Rev. 2010, 1, 5214.

[65] C. Barbé, J. Bartlett, L. Kong, K. Finnie, H. Q. Lin, M. Larkin, S. Calleja, A. Bush, G. Calleja, Adv. Mater. 2004, 16, 1959.

[66] A. Maleki, H. Kettiger, A. Schoubben, J. M. Rosenholm, V. Ambrogi, M. Hamidi, J. Controlled Release 2017, 262, 329.

[67] M. Börgardts, K. Verlinden, M. Neidhardt, T. Wöhrle, A. Herbst, S. Laschat, C. Janiak, T. J. J. Müller, RSC Adv 2016, 6, 6209.

[68] X. Du, X. Li, L. Xiong, X. Zhang, F. Kleitz, S. Z. Qiao, Biomaterials 2016, 91, 90.

[69] Y.-S. Chen, W. Frey, S. Kim, K. Homan, P. Kruizinga, K. Sokolov, S. Emelianov, Opt. Express 2010, 18, 8867.

[70] R. Alwi, S. Telenkov, A. Mandelis, T. Leshuk, F. Gu, S. Oladepo, K. Michaelian, Biomed. Opt. Express 2012, 3, 2500.

[71] O. Baber, M. Jang, D. Barber, K. Powers, Inhal. Toxicol. 2011, 23, 532.

[72] A. Guerrero-Martinez, J. Perez-Juste, L. M. Liz-Marzin, Adv. Mater. 2010, 22, 1182.

[73] Z. Sun, X. Zhou, W. Luo, Q. Yue, Y. Zhang, X. Cheng, W. Li, B. Kong, Y. Deng, D. Zhao, Nano Today 2016, 11, 464.

[74] R. Ghosh Chaudhuri, S. Paria, Chem. Rev. 2012, 112, 2373.

[75] S. A. Corr, Y. P. Rakovich, Y. K. Gun'ko, Nanoscale Res. Lett. 2008, 3, 87.

[76] T. Gulin-Sarfraz, J. Zhang, D. Desai, J. Teuho, J. Sarfraz, H. Jiang, C. Zhang, C. Sahlgren, M. Lindén, H. Gu, J. M. Rosenholm, Biomater. Sci. 2014, 2, 1750.

[77] J. Kim, J. E. Lee, J. Lee, J. H. Yu, B. C. Kim, K. An, Y. Hwang, C.-H. Shin, J.-G. Park, J. Kim, T. Hyeon, J. Am. Chem. Soc. 2006, 128, 688.

[78] Y. Chen, H. Chen, S. Zhang, F. Chen, S. Sun, Q. He, M. Ma, X. Wang, H. Wu, L. Zhang, L. Zhang, J. Shi, Biomaterials 2012, 33, 2388.

[79] T. Kim, E. Momin, J. Choi, K. Yuan, H. Zaidi, J. Kim, M. Park, N. Lee, M. T. McMahon, A. QuinonesHinojosa, J. W. M. Bulte, T. Hyeon, A. A. Gilad, J. Am. Chem. Soc. 2011, 133, 2955.

[80] Z. Xu, Y. Gao, S. Huang, P. an Ma, J. Lin, J. Fang, Dalton Trans. 2011, 40, 4846.

[81] E. von Haartman, H. Jiang, A. A. Khomich, J. Zhang, S. A. Burikov, T. A. Dolenko, J. Ruokolainen, H. Gu, O. A. Shenderova, I. I. Vlasov, J. M. Rosenholm, J. Mater. Chem. B 2013, 1, 2358.

[82] K.-J. Lin, L.-J. Chen, M. R. Prasad, C.-Y. Cheng, Adv. Mater. 2004, 16, 1845.

[83] S. H. Joo, J. Y. Park, C.-K. Tsung, Y. Yamada, P. Yang, G. A. Somorjai, Nat. Mater. 2009, 8, 126.

[84] R. I. Nooney, T. Dhanasekaran, Y. Chen, R. Josephs, A. E. Ostafin, Adv. Mater. 2002, 14, 529.

[85] R. I. Nooney, D. Thirunavukkarasu, Y. Chen, R. Josephs, A. E. Ostafin, Langmuir 2003, 19, 7628.

[86] H. Fan, J. Gabaldon, C. J. Brinker, Y.-B. Jiang, Chem. Commun. 2006, 2323.

[87] P. Botella, A. Corma, M. T. Navarro, Chem. Mater. 2007, 19, 1979.

[88] I. Gorelikov, N. Matsuura, Nano Lett. 2008, 8, 369.

[89] J. Xingmao, C. J. Brinker, J.Am.Chem.Soc. 2006, 128, 4512.

[90] J. Lai, B. P. Shah, Y. Zhang, L. Yang, K.-B. Lee, ACS Nano 2015, 9, 5234.

[91] M. Liong, B. France, K. A. Bradley, J. I. Zink, Adv. Mater. 2009, 21, 1684.

[92] M. W. Ambrogio, C. R. Thomas, Y.-L. Zhao, J. I. Zink, J. F. Stoddart, Acc. Chem. Res. 2011, 44, 903.

[93] J. M. Rosenholm, C. Sahlgren, M. Lindén, Nanoscale 2010, 2, 1870. 
[94] R. Nyffenegger, C. Quellet, J. Ricka, J. Colloid Interface Sci. 1993, 159, 150.

[95] A. Van Blaaderen, A. Vrij, Langmuir 1992, 8, 2921.

[96] S. Bonacchi, D. Genovese, R. Juris, M. Montalti, L. Prodi, E. Rampazzo, M. Sgarzi, N. Zaccheroni, Top. Curr. Chem. 2011, 300, 93.

[97] H. D. Duong, J. I. Rhee, Sens. Actuators B Chem. 2007, 124, 18.

[98] H. Mader, X. Li, S. Saleh, M. Link, P. Kele, O. S. Wolfbeis, Ann. N. Y. Acad. Sci. 2008, 1130, 218.

[99] D. Karaman, D. Desai, R. Senthilkumar, E. M. Johansson, N. Råtts, M. Odín, J. E. Eriksson, C. Sahlgren, D. M. Toivola, J. M. Rosenholm, Nanoscale Res. Lett. 2012, 7, 358.

[100] D. Desai, D. S. Karaman, N. Prabhakar, S. Tadayon, A. Duchanoy, D. M. Toivola, S. Rajput, T. Näreoja, J. M. Rosenholm, Mesoporous Biomater. 2014, 1, DOI 10.2478/mesbi-2014-0001.

[101] X. Guo, B. Guo, X. Sun, Q. Zhang, T. Shi, Chin. J. Chem. 2011, 29, 363.

[102] R. Kumar, I. Roy, T. Y. Ohulchanskyy, L. N. Goswami, A. C. Bonoiu, E. J. Bergey, K. M. Tramposch, A. Maitra, P. N. Prasad, ACS Nano 2008, 2, 449.

[103] G. Yao, L. Wang, Y. Wu, J. Smith, J. Xu, W. Zhao, E. Lee, W. Tan, Anal. Bioanal. Chem. 2006, 385, 518.

[104] S. Das, T. K. Jain, A. Maitra, J. Colloid Interface Sci. 2002, 252, 82.

[105] R. K. Sharma, S. Das, A. Maitra, J. Colloid Interface Sci. 2004, 277, 342.

[106] H. Ow, D. R. Larson, M. Srivastava, B. A. Baird, W. W. Webb, U. Wiesner, Nano Lett. 2005, 5, 113.

[107] E. Phillips, O. Penate-Medina, P. B. Zanzonico, R. D. Carvajal, P. Mohan, Y. Ye, J. Humm, M. Gonen, H. Kalaigian, H. Schoder, H. W. Strauss, S. M. Larson, U. Wiesner, M. S. Bradbury, Sci. Transl. Med. 2014, 6, 260 ra149.

[108] E. Rampazzo, L. Prodi, L. Petrizza, N. Zaccheroni, Top. Curr. Chem. 2016, 370, 1.

[109] W. Arap, R. Pasqualini, M. Montalti, L. Petrizza, L. Prodi, E. Rampazzo, N. Zaccheroni, S. Marchio, Current medicinal chemistry 2013, 20, 2195.

[110] L. D. Carlos, R. A. Sá Ferreira, R. N. Pereira, M. Assunção, V. de Zea Bermudez, J. Phys. Chem. B 2004, 108, 14924.

[111] T. Y. Ohulchanskyy, I. Roy, K.-T. Yong, H. E. Pudavar, P. N. Prasad, Wiley Interdiscip. Rev. Nanomed. Nanobiotechnol. 2010, 2, 162.

[112] D. R. Larson, H. Ow, H. D. Vishwasrao, A. A. Heikal, U. Wiesner, W. W. Webb, Chem. Mater. 2008, 20, 2677.

[113] I. Sokolov, S. Naik, Small 2008, 4, 934.

[114] E.-B. Cho, D. O. Volkov, I. Sokolov, Small 2010, 6, 2314

[115] E.-B. Cho, D. O. Volkov, I. Sokolov, Adv. Funct. Mater. 2011, 21, 3129.

[116] J. M. Rosenholm, T. Gulin-Sarfraz, V. Mamaeva, R. Niemi, E. Özliseli, D. Desai, D. Antfolk, E. von Haartman, D. Lindberg, N. Prabhakar, T. Näreoja, C. Sahlgren, Small 2016, 12, 1578.

[117] H. Chen, Z. Zhen, W. Tang, T. Todd, Y.-J. Chuang, L. Wang, Z. Pan, J. Xie, Theranostics 2013, 3, 650.

[118] R. Arppe, I. Hyppänen, N. Perälä, R. Peltomaa, M. Kaiser, C. Würth, S. Christ, U. Resch-Genger, M. Schäferling, T. Soukka, Nanoscale 2015, 7, 11746.

[119] Z. Li, Y. Zhang, Angew. Chem. Int. Ed. 2006, 45, 7732.

[120] J. Zhang, N. Prabhakar, T. Näreoja, J. M. Rosenholm, ACS Appl. Mater. Interfaces 2014, 6, 19064.

[121] J. M. Rosenholm, I. I. Vlasov, S. A. Burikov, T. A. Dolenko, O. A. Shenderova, J. Nanosci. Nanotechnol. 2015, 15, 959.

[122] A. Katiyar, S. Yadav, P. G. Smirniotis, N. G. Pinto, J. Chromatogr. A 2006, 1122, 13.

[123] S. Liu, P. Cool, O. Collart, P. Van Der Voort, E. F. Vansant, O. I. Lebedev, G. Van Tendeloo, M. Jiang, J. Phys. Chem. B 2003, 107, 10405.

[124] C.-H. Lee, S.-H. Cheng, Y.-J. Wang, Y.-C. Chen, N.-T. Chen, J. Souris, C.-T. Chen, C.-Y. Mou, C.-S. Yang, L.-W. Lo, Adv. Funct. Mater. 2009, 19, 215.

[125] D. Chamberland, Y. Jiang, X. Wang, Integr. Biol. 2010, 2, 496.

[126] W.-T. Chen, U. Mahmood, R. Weissleder, C.-H. Tung, Arthritis Res. Ther. 2005, 7, R310.

[127] M. Benezra, O. Penate-Medina, P. B. Zanzonico, D. Schaer, H. Ow, A. Burns, E. DeStanchina, V. Longo, E. Herz, S. Iyer, J. Wolchok, S. M. Larson, U. Wiesner, M. S. Bradbury, J. Clin. Invest. 2011, 121, 2768.

[128] Cherenkov, P.A., Comptes Rendus Dokl. Acad. Sci. URSS 1934, 8, 451. 
[129] P. A. Cherenkov, Phys. Rev. 1937, 52, 378.

[130] B. J. Beattie, D. L. J. Thorek, C. R. Schmidtlein, K. S. Pentlow, J. L. Humm, A. H. Hielscher, PLOS ONE 2012, 7, e31402.

[131] D. L. J. Thorek, C. C. Riedl, J. Grimm, J. Nucl. Med. 2014, 55, 95.

[132] C. Lohrmann, H. Zhang, D. L. J. Thorek, P. Desai, P. B. Zanzonico, J. O’Donoghue, C. P. Irwin, T. Reiner, J. Grimm, W. A. Weber, J. Nucl. Med. 2015, 56, 805.

[133] D. N. Pandya, R. Hantgan, M. M. Budzevich, N. D. Kock, D. L. Morse, I. Batista, A. Mintz, K. C. Li, T. J. Wadas, Theranostics 2016, 6, 698.

[134] D. L. J. Thorek, A. Ogirala, B. J. Beattie, J. Grimm, Nat Med 2013, 19, 1345.

[135] R. S. Dothager, R. J. Goiffon, E. Jackson, S. Harpstrite, D. Piwnica-Worms, PLOS ONE 2010, 5, e13300.

[136] P. T. K. Chin, M. M. Welling, S. C. J. Meskers, R. A. Valdes Olmos, H. Tanke, F. W. B. van Leeuwen, Eur. J. Nucl. Med. Mol. Imaging 2013, 40, 1283.

[137] A. Kamkaew, L. Cheng, S. Goel, H. F. Valdovinos, T. E. Barnhart, Z. Liu, W. Cai, ACS Appl. Mater. Interfaces 2016, 8, 26630.

[138] T. M. Shaffer, E. C. Pratt, J. Grimm, Nat Nano 2017, 12, 106.

[139] D. Ş. Karaman, D. Desai, J. Zhang, S. Tadayon, G. Unal, J. Teuho, J. Sarfraz, J.-H. Smått, H. Gu, T. Näreoja, J. M. Rosenholm, J. Mater. Chem. B 2016, 4, 1720.

[140] J. E. Lee, N. Lee, T. Kim, J. Kim, T. Hyeon, Acc. Chem. Res. 2011, 44, 893.

[141] J. Chen, R. Zhang, L. Han, B. Tu, D. Zhao, Nano Res. 2013, 6, 871.

[142] Y.-G. Song, H. Chang, K. Lee, Korean J. Chem. Eng. 2017, DOI 10.1007/s11814-017-0057-6.

[143] T.-H. Shin, Y. Choi, S. Kim, J. Cheon, Chem Soc Rev 2015, 44, 4501.

[144] J. Zhang, X. Li, J. M. Rosenholm, H. Gu, J. Colloid Interface Sci. 2011, 361, 16.

[145] T. Kim, E. Momin, J. Choi, K. Yuan, H. Zaidi, J. Kim, M. Park, N. Lee, M. T. McMahon, A. QuinonesHinojosa, J. W. M. Bulte, T. Hyeon, A. A. Gilad, J. Am. Chem. Soc. 2011, 133, 2955.

[146] J. Zhang, J. M. Rosenholm, H. Gu, ChemPhysChem 2012, 13, 2016.

[147] T. Gulin-Sarfraz, J. Sarfraz, D. Ş. Karaman, J. Zhang, C. Oetken-Lindholm, A. Duchanoy, J. M. Rosenholm, D. Abankwa, RSC Adv. 2014, 4, 16429.

[148] F. Stallmach, J. Kärger, C. Krause, M. Jeschke, U. Oberhagemann, J. Am. Chem. Soc. 2000, 122, 9237.

[149] Li, Yingying Shen, Yuanzhi Shao, Haoqiang He, Yunpu Tan, Xiumei Tian, Fukang Xie, Int. J. Nanomedicine 2013, 119.

[150] Y. Shao, X. Tian, W. Hu, Y. Zhang, H. Liu, H. He, Y. Shen, F. Xie, L. Li, Biomaterials 2012, 33, 6438.

[151] F. Carniato, L. Tei, A. Arrais, L. Marchese, M. Botta, Chem. - Eur. J. 2013, 19, 1421.

[152] F. Carniato, L. Tei, M. Cossi, L. Marchese, M. Botta, Chem. - Eur. J. 2010, 16, 10727.

[153] J. J. Davis, W.-Y. Huang, G.-L. Davies, 2012, 22, 22848.

[154] A. D. Sherry, P. Caravan, R. E. Lenkinski, J. Magn. Reson. Imaging 2009, 30, 1240.

[155] J. Moreau, E. Guillon, J.-C. Pierrard, J. Rimbault, M. Port, M. Aplincourt, Chem. - Eur. J. 2004, 10, 5218.

[156] E. Brucher, A. D. Sherry, Inorg. Chem. 1990, 29, 1555.

[157] A. Bianchi, L. Calabi, C. Giorgi, P. Losi, P. Mariani, P. Paoli, P. Rossi, B. Valtancoli, M. Virtuani, J. Chem. Soc. Dalton Trans. 2000, 697.

[158] X. Wang, T. Jin, V. Comblin, A. Lopez-Mut, E. Merciny, J. F. Desreux, Inorg. Chem. 1992, 31, 1095.

[159] J.-K. Hsiao, C.-P. Tsai, T.-H. Chung, Y. Hung, M. Yao, H.-M. Liu, C.-Y. Mou, C.-S. Yang, Y.-C. Chen, D.-M. Huang, Small 2008, 4, 1445.

[160] C.-P. Tsai, Y. Hung, Y.-H. Chou, D.-M. Huang, J.-K. Hsiao, C. Chang, Y.-C. Chen, C.-Y. Mou, Small 2008, $4,186$.

[161] J. L. Vivero-Escoto, K. M. L. Taylor-Pashow, R. C. Huxford, J. Della Rocca, C. Okoruwa, H. An, W. Lin, W. Lin, Small 2011, 7, 3519.

[162] M. Laprise-Pelletier, M. Bouchoucha, J. Lagueux, P. Chevallier, R. Lecomte, Y. Gossuin, F. Kleitz, M.-A. Fortin, J Mater Chem B 2015, 3, 748.

[163] R. Boiteau, T. Meade, J. Major, Nanoscape 2008, 5.

[164] Y. Chen, H. Chen, J. Shi, Adv. Mater. 2013, 25, 3144. 
[165] L. Shan, in Mol. Imaging Contrast Agent Database MICAD, National Center For Biotechnology Information (US), Bethesda (MD), 2004.

[166] R. Guillet-Nicolas, J.-L. Bridot, Y. Seo, M.-A. Fortin, F. Kleitz, Adv. Funct. Mater. 2011, 21, 4653.

[167] J. Shin, R. Anisur, M. Ko, G. Im, J. Lee, I. Lee, Angew. Chem. Int. Ed. 2009, 48, 321.

[168] R. Guillet-Nicolas, M. Laprise-Pelletier, M. M. Nair, P. Chevallier, J. Lagueux, Y. Gossuin, S. Laurent, F. Kleitz, M.-A. Fortin, Nanoscale 2013, 5, 11499.

[169] N. Hawrylak, P. Ghosh, J. Broadus, C. Schlueter, W. T. Greenough, P. C. Lauterbur, Exp. Neurol. 1993, $121,181$.

[170] J. W. M. Bulte, T. Douglas, B. Witwer, S.-C. Zhang, E. Strable, B. K. Lewis, H. Zywicke, B. Miller, P. van Gelderen, B. M. Moskowitz, I. D. Duncan, J. A. Frank, Nat. Biotechnol. 2001, 19, 1141.

[171] C. Heyn, J. A. Ronald, S. S. Ramadan, J. A. Snir, A. M. Barry, L. T. MacKenzie, D. J. Mikulis, D. Palmieri, J. L. Bronder, P. S. Steeg, T. Yoneda, I. C. MacDonald, A. F. Chambers, B. K. Rutt, P. J. Foster, Magn. Reson. Med. 2006, 56, 1001.

[172] D. L. Kraitchman, Circulation 2003, 107, 2290.

[173] I. J. M. de Vries, W. J. Lesterhuis, J. O. Barentsz, P. Verdijk, J. H. van Krieken, O. C. Boerman, W. J. G. Oyen, J. J. Bonenkamp, J. B. Boezeman, G. J. Adema, J. W. M. Bulte, T. W. J. Scheenen, C. J. A. Punt, A. Heerschap, C. G. Figdor, Nat. Biotechnol. 2005, 23, 1407.

[174] J. W. M. Bulte, D. L. Kraitchman, NMR Biomed. 2004, 17, 484.

[175] C.-W. Lu, Y. Hung, J.-K. Hsiao, M. Yao, T.-H. Chung, Y.-S. Lin, S.-H. Wu, S.-C. Hsu, H.-M. Liu, C.-Y. Mou, C.-S. Yang, D.-M. Huang, Y.-C. Chen, Nano Lett. 2007, 7, 149.

[176] J. W. M. Bulte, Am. J. Roentgenol. 2009, 193, 314.

[177] K. M. L. Taylor, J. S. Kim, W. J. Rieter, H. An, W. Lin, W. Lin, J. Am. Chem. Soc. 2008, 130, 2154.

[178] W.-I. Lin, C.-Y. Lin, Y.-S. Lin, S.-H. Wu, Y.-R. Huang, Y. Hung, C. Chang, C.-Y. Mou, J Mater Chem B 2013, 1, 639.

[179] Y.-Z. Shao, L.-Z. Liu, S. Song, R. Cao, H. Liu, C. Cui, X. Li, M.-J. Bie, L. Li, Contrast Media Mol. Imaging 2011, 6, 110.

[180] H. K. Cho, H.-J. Cho, S. Lone, D.-D. Kim, J. H. Yeum, I. W. Cheong, J. Mater. Chem. 2011, 21, 15486.

[181] F. Chen, H. Hong, Y. Zhang, H. F. Valdovinos, S. Shi, G. S. Kwon, C. P. Theuer, T. E. Barnhart, W. Cai, ACS Nano 2013, 7, 9027.

[182] F. Chen, H. Hong, S. Shi, S. Goel, H. F. Valdovinos, R. Hernandez, C. P. Theuer, T. E. Barnhart, W. Cai, 2014, 4, 5080.

[183] S. Goel, F. Chen, S. Luan, H. F. Valdovinos, S. Shi, S. A. Graves, F. Ai, T. E. Barnhart, C. P. Theuer, W. Cai, Adv. Sci. 2016, 3, 1600122.

[184] S. Goel, F. Chen, H. Hong, H. F. Valdovinos, R. Hernandez, S. Shi, T. E. Barnhart, W. Cai, ACS Appl. Mater. Interfaces 2014, 6, 21677.

[185] S. B. Lee, H. L. Kim, H.-J. Jeong, S. T. Lim, M.-H. Sohn, D. W. Kim, Angew. Chem. Int. Ed. 2013, 52, 10549.

[186] C. Denk, D. Svatunek, S. Mairinger, J. Stanek, T. Filip, D. Matscheko, C. Kuntner, T. Wanek, H. Mikula, Bioconjug. Chem. 2016, 27, 1707.

[187] E. Lipani, S. Laurent, M. Surin, L. Vander Elst, P. Leclère, R. N. Muller, Langmuir 2013, 29, 3419.

[188] Z. Zheng, A. Daniel, W. Yu, B. Weber, J. Ling, A. H. E. M?ller, Chem. Mater. 2013, 25, 4585.

[189] X. Hu, M. Wang, F. Miao, J. Ma, H. Shen, N. Jia, J Mater Chem B 2014, 2, 2265.

[190] E. S. Jang, S. Y. Lee, E.-J. Cha, I.-C. Sun, I. C. Kwon, D. Kim, Y. I. Kim, K. Kim, C.-H. Ahn, Pharm. Res. 2014, 31, 3371.

[191] Y. Chen, H. Chen, Y. Sun, Y. Zheng, D. Zeng, F. Li, S. Zhang, X. Wang, K. Zhang, M. Ma, Q. He, L. Zhang, J. Shi, Angew. Chem. Int. Ed. 2011, 50, 12505.

[192] Y. Chen, Q. Yin, X. Ji, S. Zhang, H. Chen, Y. Zheng, Y. Sun, H. Qu, Z. Wang, Y. Li, X. Wang, K. Zhang, L. Zhang, J. Shi, Biomaterials 2012, 33, 7126.

[193] J. R. Bursten, M. C. Roca, W. Yang, Y. Zhao, C. Chen, K. Savolainen, C. Gerber, H. P. Lang, K. Kataoka, Y. Krishman, H. Bayley, L. Nazar, S. Milana, L. Vandersypen, P. S. Weiss, J. Schummer, Nat. Nanotechnol. 2016, 11, 828.

[194] A. Uhlir, Bell Syst Tech 1956, 35, DOI 10.1002/j.1538-7305.1956.tb02385.x. 
[195] D. R. Turner, J. Electrochem. Soc. 1958, 105, 402.

[196] L. T. Canham, Appl Phys Lett 1990, 57, DOI 10.1063/1.103561.

[197] V. Lehmann, U. Gösele, Appl. Phys. Lett. 1991, 58, 856.

[198] A. Saar, J. Nanophotonics 2009, 3, 032501.

[199] L. T. Canham, Adv. Mater. 1995, 7, 1033.

[200] E. J. Anglin, L. Cheng, W. R. Freeman, M. J. Sailor, Inorg. Nanoparticles Drug Deliv. 2008, 60, 1266.

[201] H. A. Santos, L. M. Bimbo, V. P. Lehto, A. J. Airaksinen, J. Salonen, J. Hirvonen, Curr Drug Discov Technol 2011, 8, 228.

[202] J. Salonen, V.-P. Lehto, Porous Inorg. Mater. Biomed. Appl. 2008, 137, 162.

[203] B. Godin, E. Tasciotti, X. Liu, R. E. Serda, M. Ferrari, Acc Chem Res 2011, 44, 979.

[204] R. E. Serda, B. Godin, E. Blanco, C. Chiappini, M. Ferrari, Biochim Biophys Acta 2011, 1810, 317.

[205] C. Chiappini, E. Tasciotti, J. R. Fakhoury, D. Fine, L. Pullan, Y.-C. Wang, L. Fu, X. Liu, M. Ferrari, ChemPhysChem 2010, 11, 1029.

[206] V. Petrova-Koch, T. Muschik, Appl. Phys. Lett. 1992, 61, 943.

[207] J. Salonen, E. Laine, L. NiinistÃ ף, J. Appl. Phys. 2002, 91, 456.

[208] J. Salonen, M. Björkqvist, E. Laine, L. Niinistö, Appl. Surf. Sci. 2004, 225, 389.

[209] M. Arroyo-Hernández, R. J. Martín-Palma, J. Pérez-Rigueiro, J. P. García-Ruiz, J. L. García-Fierro, J. M. Martínez-Duart, Curr. Trends Nanosci. - Mater. Appl. Proc. Symp. E-MRS Spring Meet. 2003 2003, 23, 697.

[210] R. Boukherroub, J. T. C. Wojtyk, D. D. M. Wayner, D. J. Lockwood, J. Electrochem. Soc. 2002, 149, H59.

[211] C.-F. Wang, E. M. Mäkilä, C. Bonduelle, J. Rytkönen, J. Raula, S. Almeida, A. Närvänen, J. J. Salonen, S. Lecommandoux, J. T. Hirvonen, H. A. Santos, ACS Appl. Mater. Interfaces 2015, 7, 2006.

[212] B. Godin, J. Gu, R. E. Serda, R. Bhavane, E. Tasciotti, C. Chiappini, X. Liu, T. Tanaka, P. Decuzzi, M. Ferrari, J. Biomed. Mater. Res. A 2010, 94A, 1236.

[213] E. Tasciotti, B. Godin, J. O. Martinez, C. Chiappini, R. Bhavane, X. Liu, M. Ferrari, Mol Imaging 2011, 10, 56.

[214] S. Srinivasan, J. F. Alexander, W. H. Driessen, F. Leonard, H. Ye, X. Liu, W. Arap, R. Pasqualini, M. Ferrari, B. Godin, J. Mater. Chem. B 2013, 1, 5218.

[215] B. Xia, W. Zhang, J. Shi, S. Xiao, ACS Appl. Mater. Interfaces 2013, 5, 11718.

[216] A. Gizzatov, C. Stigliano, J. S. Ananta, R. Sethi, R. Xu, A. Guven, M. Ramirez, H. Shen, A. Sood, M. Ferrari, L. J. Wilson, X. Liu, P. Decuzzi, Cancer Lett 2014, 352, 97.

[217] H. A. Santos, L. M. Bimbo, B. Herranz, M.-A. Shahbazi, J. Hirvonen, J. Salonen, J. Mater. Res. 2013, 28, 152.

[218] E. C. Wu, J. S. Andrew, L. Cheng, W. R. Freeman, L. Pearson, M. J. Sailor, Biomaterials 2011, 32, 1957.

[219] S. N. A. Jenie, S. E. Plush, N. H. Voelcker, Pharm. Res. 2016, 33, 2314.

[220] L. Gu, D. J. Hall, Z. Qin, E. Anglin, J. Joo, D. J. Mooney, S. B. Howell, M. J. Sailor, Nat Commun 2013, 4, 2326.

[221] J. Joo, X. Liu, V. R. Kotamraju, E. Ruoslahti, Y. Nam, M. J. Sailor, ACS Nano 2015, 9, 6233.

[222] L. A. Osminkina, V. A. Sivakov, G. A. Mysov, V. A. Georgobiani, C. Natashina Ucapital A, F. Talkenberg, V. V. Solovyev, A. A. Kudryavtsev, V. Y. Timoshenko, Nanoscale Res Lett 2014, 9, 463.

[223] C. F. Wang, M. P. Sarparanta, E. M. Mäkilä, M. L. Hyvonen, P. M. Laakkonen, J. J. Salonen, J. T. Hirvonen, A. J. Airaksinen, H. A. Santos, Biomaterials 2015, 48, 108.

[224] C. M. Lundquist, C. Loo, I. M. Meraz, J. De La Cerda, X. Liu, R. E. Serda, Med Sci Basel 2014, 2, 51.

[225] A. Gizzatov, J. Key, S. Aryal, J. Ananta, A. Cervadoro, A. L. Palange, M. Fasano, C. Stigliano, M. Zhong, D. Di Mascolo, A. Guven, E. Chiavazzo, P. Asinari, X. Liu, M. Ferrari, L. J. Wilson, P. Decuzzi, Adv Funct Mater 2014, 24, 4584.

[226] T. Nissinen, S. Nakki, H. Laakso, D. Kuciauskas, A. Kaupinis, M. I. Kettunen, T. Liimatainen, M. Hyvonen, M. Valius, O. Grohn, V. P. Lehto, ACS Appl Mater Interfaces 2016, 8, 32723.

[227] M. C. Cassidy, H. R. Chan, B. D. Ross, P. K. Bhattacharya, C. M. Marcus, Nat. Nanotechnol. 2013, 8, 363. 
[228] L. M. Bimbo, M. Sarparanta, H. A. Santos, A. J. Airaksinen, E. Mäkilä, T. Laaksonen, L. Peltonen, V.-P. Lehto, J. Hirvonen, J. Salonen, ACS Nano 2010, 4, 3023.

[229] M. Sarparanta, L. M. Bimbo, J. Rytkonen, E. Mäkilä, T. J. Laaksonen, P. Laaksonen, M. Nyman, J. Salonen, M. B. Linder, J. Hirvonen, H. A. Santos, A. J. Airaksinen, Mol Pharm 2012, 9, 654.

[230] M. P. Sarparanta, L. M. Bimbo, E. M. Mäkilä, J. J. Salonen, P. H. Laaksonen, A. M. K. Helariutta, M. B. Linder, J. T. Hirvonen, T. J. Laaksonen, H. A. Santos, A. J. Airaksinen, Biomaterials 2012, 33, 3353.

[231] D. Liu, E. Mäkilä, H. Zhang, B. Herranz, M. Kaasalainen, P. Kinnari, J. Salonen, J. Hirvonen, H. A. Santos, Adv. Funct. Mater. 2013, 23, 1893.

[232] A. M. Kallinen, M. P. Sarparanta, D. Liu, E. M. Mäkilä, J. J. Salonen, J. T. Hirvonen, H. A. Santos, A. J. Airaksinen, Mol Pharm 2014, 11, 2876.

[233] C. Tu, X. Ma, A. House, S. M. Kauzlarich, A. Y. Louie, ACS Med. Chem. Lett. 2011, 2, 285.

[234] J. Rytkönen, R. Miettinen, M. Kaasalainen, V.-P. Lehto, J. Salonen, Närvänen, J. Nanomater. 2012, 2012, e896562.

[235] M. P. A. Ferreira, S. Ranjan, A. M. R. Correia, E. M. Mäkilä, S. M. Kinnunen, H. Zhang, M.-A. Shahbazi, P. V. Almeida, J. J. Salonen, H. J. Ruskoaho, A. J. Airaksinen, J. T. Hirvonen, H. A. Santos, Biomaterials 2016, 94, 93.

[236] M. P. A. Ferreira, S. Ranjan, S. Kinnunen, A. Correia, V. Talman, E. Mäkilä, B. Barrios-Lopez, M. Kemell, V. Balasubramanian, J. Salonen, J. Hirvonen, H. Ruskoaho, A. J. Airaksinen, H. A. Santos, Small 2017, 13, n/a.

[237] C. F. Wang, M. P. Sarparanta, E. M. Mäkilä, M. L. Hyvönen, P. M. Laakkonen, J. J. Salonen, J. T. Hirvonen, A. J. Airaksinen, H. A. Santos, Biomaterials 2015, 48, 108.

[238] O. Keinänen, E. M. Mäkilä, R. Lindgren, H. Virtanen, H. Liljenbäck, V. Oikonen, M. Sarparanta, C. Molthoff, A. D. Windhorst, A. Roivainen, J. J. Salonen, A. J. Airaksinen, ACS Omega 2017, 2, 62.

[239] O. Keinänen, X.-G. Li, N. K. Chenna, D. Lumen, J. Ott, C. F. M. Molthoff, M. Sarparanta, K. Helariutta, T. Vuorinen, A. D. Windhorst, A. J. Airaksinen, ACS Med. Chem. Lett. 2016, 7, 62. 


\section{Figure captions:}

Figure 1. Design options for theranostic nanoparticles. Physical properties, surface modifications and composition of porous and non-porous nanoparticles can be tuned in order to make them powerful tools for imaging. For instance, the size and morphology of the particles can be tuned to enhance their interaction with cells and their permeability across biological barriers; reactive groups can be added on the particle surface for the attachment of different organic moieties, biomolecules or nanoscopic imaging agents; and the incorporation of MRI, optical, or nuclear imaging agents and therapeutic payloads inside the carrier structure can be realized.

Figure 2. The microemulsion synthesis of silica particles both under basic and acidic conditions. Reprinted with permission from ref. 59. Copyrights 2007 American Chemical Society.

Figure 3. Formation of mesoporous silica materials by structure-directing agents: a) true liquid-crystal template mechanism, b) cooperative liquid-crystal template mechanism. Reprinted with permission from ref. 62. Copyrights 2006 Wiley-VCH Verlag GmbH \& Co.

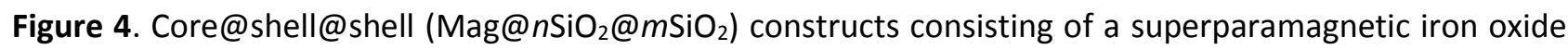
core (Mag), a non-porous silica inner shell $\left(n \mathrm{SiO}_{2}\right)$ separating the core from the mesoporous silica outer shell $\left(m \mathrm{SiO}_{2}\right)$. A) TEM image showing the morphology and structure of the magnetic cores coated with non-porous and porous silica layers; B) The effect of surface functionalization and an external magnetic field on cellular labeling of $\mathrm{Mag@nSiO} \mathrm{O}_{2} @ m \mathrm{SiO}_{2}$ particles was studied, whereby the cellular uptake of the surface functionalized particles could readily be further enhanced by an applied magnetic field; C) Fluorescence intensities, of similarly FITC-labeled magnetic core particles or magnetic core@shell particles with increasing number of silica layers, were measured in HEPES buffer (pH 7.2) between 510 and $610 \mathrm{~nm}$ using $488 \mathrm{~nm}$ excitation. The importance of the thicker non-porous silica barrier (towards the magnetite core) for the 
fluorescence intensity is demonstrated; D) MRI comparison of labeled cells as a function of treatment time with and without the presence of an external magnetic field; E) The $1 / T_{2}$ values, derived from the corresponding images, as a function of various labeling time points. The trend, which these results show, confirm the positive effect of the magnetic field on the cellular uptake. When a magnetic field was applied for $30 \mathrm{~min}$, an almost 6 -fold increase in $1 / T_{2}$ was found compared to the control cells; F) Cellular uptake of PEl-surface functionalized $\mathrm{Mag} @ n \mathrm{SiO}_{2} @ m \mathrm{SiO}_{2}$ particles as a function of time, determined by flow cytometry both based on fluorescence and reflection. A magnetic field can readily enhance the uptake of the particles. The magnetically enhancement is more pronounced for short time intervals, but even after $3 \mathrm{~h}$ of incubation a clear difference is seen. This simultaneously demonstrates that results obtained from fluorescence and reflection based measurements are comparable if no particle aggregation occur, which in this case was avoided by appropriate surface functionalization with PEI. Adapted with permission from ref. 76. Copyrights 2014 Royal Society of Chemistry.

Figure 5. In vivo imaging of fluorophore-loaded MSN labeled MDA-MB-231 breast cancer cells xenografted in mice. A) The cells were visible for a period of 32 days. B, C) Ex vivo imaging of isolated organs verified tumor labeling and allowed for the detection of metastatic colonies. Reprinted with permission from ref. 116. Copyrights 2016 John Wiley and Sons.

Figure 6. Results of Kamkaew et al., 2016, showing an improved therapeutic effect in 4T1 mammary adenocarcinoma xenografts with Cherenkov radiation activation of the photosensitizer Chlorin e6 loaded in hollow mesoporous silica nanoparticles $\left.\left({ }^{89} \mathrm{Zr}\right] \mathrm{HMSN}-\mathrm{Ce} 6\right)$ with the incorporation of the positron-emitting radionuclide ${ }^{89} \mathrm{Zr}$. Reprinted with permission from ref. 137. Copyrights 2016 American Chemical Society.

Figure 7. The MSN preparation route influence on the relaxometric properties. Transmission electron microscope image and schematic representation of Gd-DOTA-MSNs (66.3 $\pm 6.6 \mathrm{~nm}$ ) prepared using (a) 'Short delay' co-condensation, where functionalities are internalised deeply in the structure $\left(r_{1}=17.14 \pm 0.49 \mathrm{mM}^{-1} \mathrm{~s}\right.$ 
$\left.{ }^{1}\right)$, (b) 'Long delay' co-condensation, where functionalities are internalised nearer to the porous openings $\left(r_{1}=33.57 \pm 1.29 \mathrm{mM}^{-1} \mathrm{~s}^{-1}\right)$ and (c) post-grafting, where functionalities are loaded on external surfaces $\left(r_{1}=10.77 \pm 0.22 \mathrm{mM}^{-1} \mathrm{~s}^{-1}\right)$. (d) Post-grafted Gd-DOTA-non-porous silica nanoparticles $\left(r_{1}=9.56 \pm 0.47 \mathrm{mM}^{-1} \mathrm{~s}^{-1}\right)$. The chart displays $r_{1}$ relaxivities of corresponding nanoparticle samples, measured at 7 T. Reprinted with permission from ref. 153. Copyrights 2012 Royal Society of Chemistry.

Figure 8. In vivo tumor vasculature targeting. Representative tumor-bearing coronal slices from serial PET scans. Targeted group: [ $\left.{ }^{89} \mathrm{Zr}\right] \mathrm{bMSN}-\mathrm{PEG5k}-\mathrm{TRC} 105$; non-targeted group: $\left[{ }^{89} \mathrm{Zr}\right.$ ]bMSN-PEG5k and blocking group: [ ${ }^{89} \mathrm{Zr}$ ]bMSN-PEG5k-TRC105 (with a pre-injected blocking dose of TRC105). Yellow circles indicate the location of 4T1 breast tumor. Reprinted with permission from ref. 183. Copyrights 2016 John Wiley and Sons.

Figure 9. Overview of the versatility and characteristics of a multimodal nanoprobe with theranostic potential. (a) Schematic illustration of the microstructure and structure-related theranostic functions of the HMCNs designed by Chen et al. (b) TEM image of HMCNs (inset: STEM image with scale bar = $100 \mathrm{~nm}$ ); (c-f) Elemental mapping of $\mathrm{Si}(\mathrm{c}), \mathrm{O}(\mathrm{d})$ and $\mathrm{Mn}(\mathrm{e})$ in HMCNs (f: color-merged image of $\mathrm{c}$, $\mathrm{d}$ and e). Reprinted with permission from ref. 192. Copyrights 2012 Elsevier.

Figure 10. Preparation of PSi particles starts with the electrochemical etching of crystalline silicon wafers in an ethanolic solution of hydrofluoric acid, whereby the porous layer grows in the direction of the etching current $(E)$. The degree of porosity and the thickness of the porous layer can be finely controlled by the conditions of the electrochemical etch. The porous layer is lift off the crystalline Si substrate by an abrupt increase in the etching current, and the resulting PSi reduced to nano- and microparticles typically by ball milling and ultrasonic fracture followed by sieving and ultracentrifugation to isolate the desired size fraction.

Figure 11. Excitation-based fluorescence imaging of luminescent porous silicon nanoparticles (LPSiNP) in cells and both in vivo and ex vivo in MDA-MB-231 tumor-bearing mice in a pioneering study by Park et al. (2009) 
illustrates the potential of the material for the generation of optical imaging probes with simultaneous drug delivery capability. Reprinted with permission from ref. 53. Copyrights 2009 Nature Publishing Group.

Figure 12. MRI T2* map of a rat liver before and $40 \mathrm{~min}$ after the $2 \mathrm{~mL}, 0.5 \mathrm{mg} / \mathrm{mL}$ injections of MaPSi or DPEG-MaPSi nanoparticles. The liver is circled with a black line, the white triangle designates the cardiac part of the stomach, and the black asterisk shows the spinal cord. Subcutaneous fat is seen as longer relaxation times. The four spots above the body of the rat are from the water-heated pad. Reprinted with permission from ref. 226. Copyrights 2016 American Chemical Society.

Figure 13. Representative sagittal SPECT/CT images showing biodistribution of intravenously administered PEGylated $\left[{ }^{111} \mathrm{In}\right]$ UnTHCPSi nanoparticles at 10 min time point in rat with induced myocardial infraction. $\left[{ }^{111} \mathrm{In}\right]$ Un-P-D-ANP NPs were targeted to the infracted myocardium by using an ANP peptide. SUV in heart the control NPs and [111 In]Un-P-D-ANP NPs at $10 \mathrm{~min}, 20 \mathrm{~min}$ and $4 \mathrm{~h}$ time points. Values are represented as mean \pm s.d. $(n=4)$. Adapted with permission from ref. 236. Copyrights 2017 John Wiley and Sons.

Figure 14. Pretargeted PET imaging of TCO-PSi NPs in mice. A. The TCO-PSi NPs were injected 15 minutes before injection of the tracer $\left[{ }^{18} \mathrm{~F}\right]$ TAF. PET imaging was performed 60 minutes after injection of the tracer. B. The TCO-PSi NPs were traced in spleen (delineated by a box). Some accumulation also in liver was observed. The observed high levels of radioactivity in gall bladder, intestines and urinary bladder are caused by elimination of the $\left[{ }^{18} \mathrm{~F}\right]$ TAF and its radioactive metabolites. C. The time-activity-curves (TAC) revealed that the IEDDA reaction was fast, with high radioactivity accumulation in spleen already during the first 10 minutes p.i. The cycloaddition product was stable and no significant decrease in radioactivity levels in spleen was observed during the 120 minutes observation time. Adapted with permission from ref. 238. Copyrights 2017 American Chemical Society. 


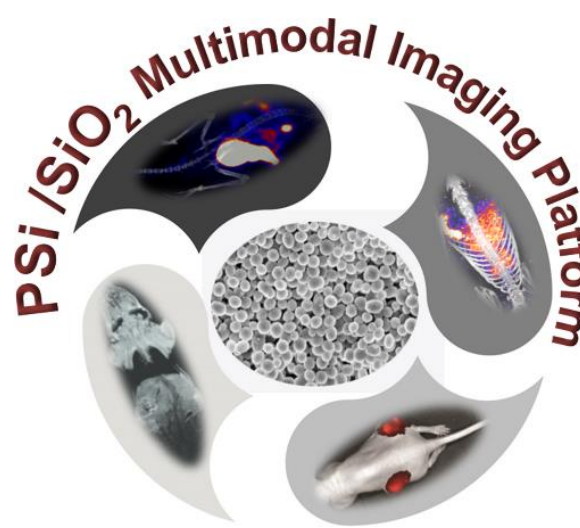

Silica $\left(\mathrm{SiO}_{2}\right)$ and silicon $(\mathrm{Si})$ are excellent material platforms for the generation of multimodality imaging probes for diagnostic and nanotheranostic applications. Both being versatile materials with multifunctional properties they can be extensively used for the creation of novel drug delivery systems for site-specific nanotherapeutic delivery. When developed into multimodality imaging probes, they are excellent tools for the investigation of biological interactions of the nanotherapeutics in the physiological environment in vivo. 